\title{
REPRESENTATIONS OF ÉTALE GROUPOIDS ON $L^{p}$-SPACES
}

\author{
EUSEBIO GARDELLA AND MARTINO LUPINI
}

\begin{abstract}
For $p \in(1, \infty)$, we study representations of étale groupoids on $L^{p}$-spaces. Our main result is a generalization of Renault's disintegration theorem for representations of étale groupoids on Hilbert spaces. We establish a correspondence between $L^{p}$-representations of an étale groupoid $G$, contractive $L^{p}$-representations of $C_{c}(G)$, and tight regular $L^{p}$-representations of any countable inverse semigroup of open slices of $G$ that is a basis for the topology of $G$. We define analogs $F^{p}(G)$ and $F_{\text {red }}^{p}(G)$ of the full and reduced groupoid $\mathrm{C}^{*}$-algebras using representations on $L^{p}$-spaces. As a consequence of our main result, we deduce that every contractive representation of $F^{p}(G)$ or $F_{\text {red }}^{p}(G)$ is automatically completely contractive. Examples of our construction include the following natural families of Banach algebras: discrete group $L^{p}$-operator algebras, the analogs of Cuntz algebras on $L^{p}$-spaces, and the analogs of AF-algebras on $L^{p}$-spaces. Our results yield new information about these objects: their matricially normed structure is uniquely determined. More generally, groupoid $L^{p}$-operator algebras provide analogs of several families of classical $\mathrm{C}^{*}$-algebras, such as Cuntz-Krieger $\mathrm{C}^{*}$-algebras, tiling $\mathrm{C}^{*}$-algebras, and graph $\mathrm{C}^{*}$-algebras.
\end{abstract}

\section{Contents}

1. Introduction

2. Borel Bundles of Banach spaces

3. Banach representations of étale groupoids 20

4. Representations of inverse semigroups on $L^{p_{\text {-spaces }}} 33$

5. Disintegration of representations 40

6. $L^{p}$-operator algebras of étale groupoids 44

7. Examples: analogs of Cuntz algebras and AF-algebras 52

8. Concluding remarks and outlook 58

References 5

2000 Mathematics Subject Classification. Primary 47L10, 22A22; Secondary 46H05.

Key words and phrases. Groupoid, Banach bundle, $L^{p}$-space, $L^{p}$-operator algebra, Cuntz algebra.

Eusebio Gardella was supported by the US National Science Foundation through Grant DMS-1101742. Martino Lupini was supported by the York University Elia Scholars Program. This work was completed when the authors were attending the Thematic Program on Abstract Harmonic Analysis, Banach and Operator Algebras at the Fields Institute. The hospitality of the Fields Institute is gratefully acknowledged. 


\section{INTRODUCTION}

Groupoids are a natural generalization of groups, where the operation is no longer everywhere defined. Succinctly, a groupoid can be defined as a small category where every arrow is invertible, with the operations being composition and inversion of arrows. A groupoid is called locally compact when it is endowed with a (not necessarily Hausdorff) locally compact topology compatible with the operations; see [26. Any locally compact group is in particular a locally compact groupoid. More generally, one can associate to a continuous action of a locally compact group on a locally compact Hausdorff space the corresponding action groupoid as in [23]. This allows one to regard locally compact groupoids as a generalization of topological dynamical systems.

A particularly important class of locally compact groupoids are those where the operations are local homeomorphisms. These are the so-called étale - or $r$-discrete [37-groupoids, and constitute the groupoid analog of actions of discrete groups on locally compact spaces. In fact, they can be described in terms of partial actions of inverse semigroups on locally compact spaces; see [10]. Alternatively, one can characterize étale groupoids as the locally compact groupoids having an open basis of slices, i.e. sets where the source and range maps are injective [10, Section 3]. In the étale case, the set of all open slices is an inverse semigroup.

The representation theory of étale groupoids on Hilbert spaces has been intensively studied since the seminal work of Renault [37]; see the monograph [26]. A representation of an étale groupoid $G$ on a Hilbert space is an assignment $\gamma \mapsto T_{\gamma}$ of an invertible isometry $T_{\gamma}$ between Hilbert spaces to any element $\gamma$ of $G$. Such an assignment is required to respect the algebraic and measurable structure of the groupoid. The fundamental result of [37] establishes a correspondence between the representations of an étale groupoid $G$ and the nondegenerate $I$-norm contractive representations of $C_{c}(G)$. (The $I$-norm on $C_{c}(G)$ is the analogue of the $L^{1}$-norm for discrete groups. When $G$ is Hausdorff, $C_{c}(G)$ is just the space of compactly-supported continuous functions on $G$. The non-Hausdorff case is more subtle; see [10, Definition 3.9].) Moreover, such a correspondence is compatible with the natural notions of equivalence for representations of $G$ and $C_{c}(G)$. In turn, nondegenerate representations of $C_{c}(G)$ correspond to tight regular representations of any countable inverse semigroup $\Sigma$ of open slices of $G$ that is a basis for the topology. Again, such correspondence preserves the natural notions of equivalence for representations of $C_{c}(G)$ and $\Sigma$. Tightness is a nondegeneracy condition introduced by Exel in [10, Section 11]. In the case when the set $G^{0}$ of objects of $G$ is compact and zero-dimensional, the one can take $\Sigma$ to be the inverse semigroup of compact open slices of $G$. In this case the semilattice $E(\Sigma)$ of idempotent elements of $\Sigma$ is the Boolean algebra of clopen subsets of $G^{0}$, and a representation of $G$ is tight if and only if its restriction to $E(\Sigma)$ is a Boolean algebra homomorphism. 
In this paper, we show how an important chapter in the theory of $\mathrm{C}^{*}$ algebras admits a natural generalization to algebras of operators on $L^{p_{-}}$ spaces, perfectly mirroring the Hilbert space case. We prove that the correspondences described in the paragraph above directly generalize when one replaces representations on Hilbert spaces with representations on $L^{p}$-spaces for some Hölder exponent $p$ in $(1, \infty)$. For $p=2$, one recovers Renault's and Exel's results. Interestingly, the proofs for $p=2$ and $p \neq 2$ differ drastically. The methods when $p \neq 2$ are based on the characterization of invertible isometries of $L^{p}$-spaces stated by Banach in [2]. (The first available proof is due to Lamperti [19, hence the name Banach-Lamperti theorem.)

Following [32, 25, 9] we say that a representation of a matricially normed algebra $A$ on $L^{p}(\lambda)$ is $p$-completely contractive if all its amplifications are contractive when the algebra of $n \times n$ matrices of bounded linear operators on $L^{p}(\lambda)$ is identified with the algebra of bounded linear operators on $\lambda \times c_{n}$. (Here and in the following, $c_{n}$ denotes the counting measure on $n$ points.) If $G$ is an étale groupoid, then the identification between $M_{n}\left(C_{c}(G)\right)$ and $C_{c}\left(G_{n}\right)$ for a suitable amplification $G_{n}$ of $G$ defines matricial norms on the algebra $C_{c}(G)$. As a corollary of our analysis a contractive representation of $C_{c}(G)$ on an $L^{p}$-space is automatically $p$-completely contractive.

In the case of Hilbert space representations, the universal object associated to $C_{c}(G)$ is the groupoid $\mathrm{C}^{*}$-algebra $C^{*}(G)$, as defined in 26 , Chapter $3]$. One can also define a reduced version $C_{\text {red }}^{*}(G)$ (see [26, pages 108-109]), that only considers representations of $C_{c}(G)$ that are induced-in the sense of Rieffel [26, Appendix D] — from a Borel probability measure on the space of objects of $G$. Amenability of the groupoid $G$ implies that the canonical surjection from $C^{*}(G)$ to $C_{\mathrm{red}}^{*}(G)$ is an isomorphism. In the case when $G$ is a countable discrete group, these objects are the usual full and reduced group $\mathrm{C}^{*}$-algebras.

A similar construction can be performed for an arbitrary $p$ in $(1, \infty)$, and the resulting universal objects are the full and reduced groupoid $L^{p}$-operator algebras $F^{p}(G)$ and $F_{\text {red }}^{p}(G)$ of $G$. When $G$ is a countable discrete group, these are precisely the full and reduced group $L^{p}$-operator algebras of $G$ as defined in [28; see also [12. When $G$ is the groupoid associated with a Bratteli diagram as in [38, Section 2.6], one obtains the spatial $L^{p}$-analog of an $\mathrm{AF} \mathrm{C}^{*}$-algebra; see 31 . (The $L^{p}$-analogs of $\mathrm{UHF} \mathrm{C}^{*}$-algebras are considered in [30, 29].) When $G$ is one of the Cuntz groupoids defined in [38, Section 2.5], one obtains the $L^{p}$-analogs of the corresponding Cuntz algebra from [27, 30, 29].

More generally, this construction provides several new examples of $L^{p_{-}}$ analogs of "classical" $\mathrm{C}^{*}$-algebras, such as Cuntz-Krieger algebras, graph algebras, and tiling $\mathrm{C}^{*}$-algebras (all of which can be realized as groupoid $\mathrm{C}^{*}$-algebras for a suitable étale groupoid; see [18] and [26]). It is worth mentioning here that there seems to be no known example of a nuclear $\mathrm{C}^{*}$ algebra that cannot be described as the enveloping $\mathrm{C}^{*}$-algebra of a locally compact groupoid. 
We believe that this point of view is a contribution towards clarifying what are the well-behaved representations of algebraic objects - such as the Leavitt algebras, Bratteli diagrams, or graphs - on $L^{p}$-spaces. In [27, 30, 29], several characterizations are given for well behaved representations of Leavitt algebras and stationary Bratteli diagrams. The fundamental property considered therein is the uniqueness of the norm that they induce. The groupoid approach shows that these representations are precisely those coming from representations of the associated groupoid or, equivalently, its inverse semigroup of open slices.

Another upshot of the present work is that the groupoid $L^{p}$-operator algebras $F^{p}(G)$ and $F_{\text {red }}^{p}(G)$ satisfy an automatic $p$-complete contractivity property for contractive homomorphisms into other $L^{p}$-operator algebras. In fact, $F^{p}(G)$ and $F_{\text {red }}^{p}(G)$ have canonical matrix norms. Such matrix norm structure satisfies the $L^{p}$-analog of Ruan's axioms for operator spaces as defined in [9, Subsection 4.1], building on [32, 25]. Using the terminology of [9, Subsection 4.1], this turns the algebras $F^{p}(G)$ and $F_{\text {red }}^{p}(G)$ into $p$ operator systems such that the multiplication is $p$-completely contractive. It is a corollary of our main results that any contractive representation of these algebras on an $L^{p}$-space is automatically $p$-completely contractive. As a consequence the matrix norms on $F^{p}(G)$ and $F_{\text {red }}^{p}(G)$ are uniquely determined - as it is the case for $\mathrm{C}^{*}$-algebras.

It is still not clear what are the well-behaved algebras of operators on $L^{p}$-spaces. Informally speaking, these should be the $L^{p}$-operator algebras that behave like $\mathrm{C}^{*}$-algebras. The results in this paper provide strong evidence that $L^{p}$-operator algebras of the form $F^{p}(G)$ and $F_{\text {red }}^{p}(G)$ for some étale groupoid $G$, indeed behave like $C^{*}$-algebras. Beside having the automatic complete contractiveness property for contractive representations on $L^{p}$-spaces, another property that $F^{p}(G)$ and $F_{\text {red }}^{p}(G)$ share with $\mathrm{C}^{*}$-algebras is being generated by spatial partial isometries as defined in [27]. These are the partial isometries whose support and range idempotents are hermitian operators in the sense of $\left[22\right.$; see also 3 . (In the $\mathrm{C}^{*}$-algebra case, the hermitian idempotents are precisely the orthogonal projections.) In particular, this property forces the algebra to be a $\mathrm{C}^{*}$-algebra in the case $p=2$. (A stronger property holds for unital $\mathrm{C}^{*}$-algebras, namely being generated by invertible isometries; see [4, Theorem II.3.2.16]. As observed by Chris Phillips, this property turns out to fail for some important examples of algebras of operators on $L^{p}$-spaces, such as the $L^{p}$-analog of the Toeplitz algebra.)

The present work indicates that the properties of being generated by spatial partial isometries, and having automatic complete contractiveness for representations on $L^{p}$-spaces, are very natural requirements for an $L^{p_{-}}$ operator algebra to behave like a $\mathrm{C}^{*}$-algebra. We believe that the results of this paper are a step towards a successful identification of those properties that characterize the class of "well behaved" $L^{p}$-operator algebras. 
1.1. Notation. We denote by $\omega$ the set of natural numbers including 0 . An element $n \in \omega$ will be identified with the set $\{0,1, \ldots, n-1\}$ of its predecessors. (In particular, 0 is identified with the empty set.) We will therefore write $j \in n$ to mean that $j$ is a natural number and $j<n$.

For $n \in \omega$ or $n=\omega$, we denote by $c_{n}$ the counting measure on $n$. We denote by $\mathbb{Q}(i)^{\oplus \omega}$ the set of all sequences $\left(\alpha_{n}\right)_{n \in \omega}$ of complex numbers in $\mathbb{Q}(i)$ such that $\alpha_{n}=0$ for all but finitely many indices $n \in \omega$.

All Banach spaces will be reflexive, and will be endowed with a (Schauder) basis. Recall that a basis $\left(b_{n}\right)_{n \in \omega}$ of a Banach space $Z$ is said to be boundedly complete if $\sum_{n \in \omega} \lambda_{n} b_{n}$ converges in $Z$ whenever $\sup _{n \in \omega}\left\|\sum_{j \in n} \lambda_{j} b_{j}\right\|<\infty$. By [7, Theorem 7.4], every basis of a reflexive Banach space is boundedly complete.

All Borel spaces will be standard. For a standard Borel space $X$, we denote by $B(X)$ the space of complex-valued bounded Borel functions on $X$, and by $\mathcal{B}(X)$ the $\sigma$-algebra of Borel subsets of $X$.

For a Borel measure $\mu$ on a standard Borel space $X$, we denote by $\mathcal{B}_{\mu}$ the measure algebra of $\mu$. This is the quotient of the Boolean algebra $\mathcal{B}(X)$ of Borel subsets of $X$ by the ideal of $\mu$-null Borel subsets. By [17, Exercise 17.44] $\mathcal{B}_{\mu}$ is a complete Boolean algebra. The characteristic function of a set $F$ will be denoted by $\chi_{F}$.

Given a measure space $(X, \mu)$ and a Hölder exponent $p \in(1, \infty)$, we will denote the Lebesgue space $L^{p}(X, \mu)$ simply by $L^{p}(\mu)$. Recall that $L^{p}(\mu)$ is separable precisely if there is a $\sigma$-finite Borel measure $\lambda$ on a standard Borel space such that $L^{p}(\lambda)$ is isometrically isomorphic to $L^{p}(\mu)$. Moreover there exists $n \in \omega \cup\{\omega\}$ such that $\lambda$ is Borel-isomorphic to $\left([0,1] \times n, \nu \times c_{n}\right)$, where $\nu$ is the Lebesgue measure on $[0,1]$. The push-forward of a measure $\mu$ under a function $\phi$ will be denote by $\phi_{*} \mu$ or $\phi_{*}(\mu)$.

If $X$ and $Z$ are Borel spaces, we say that $Z$ is fibred over $X$ if there is a Borel surjection $q: Z \rightarrow X$. In this case, we call $q$ the fiber map. A section of $Z$ is a map $\sigma: X \rightarrow Z$ such that $q \circ \sigma$ is the identity map of $X$. For $x \in X$, we denote the value of $\sigma$ at $x$ by $\sigma_{x}$, and the fiber $q^{-1}(\{x\})$ over $x$ is denoted by $Z_{x}$. If $Z^{(0)}$ and $Z^{(1)}$ are Borel spaces fibred over $X$ via fiber maps $q^{(0)}$ and $q^{(1)}$ respectively, then their fiber product $Z^{(0)} * Z^{(1)}$ is the Borel space fibred over $X$ defined by

$$
Z^{(0)} * Z^{(1)}=\left\{\left(z^{(0)}, z^{(1)}\right): p^{(0)}\left(z^{(0)}\right)=p^{(1)}\left(z^{(1)}\right)\right\} .
$$

If $E$ and $F$ are Banach spaces, we will denote by $B(E, F)$ the Banach space of bounded linear maps from $E$ to $F$. When $E=F$, we abbreviate $B(E, E)$ to just $B(E)$. Despite the apparent notational conflict with the set of Borel functions $B(X)$ on a measurable space $X$, confusion will be unlikely to arise, and it should always be clear from the context what $B(\cdot)$ means.

Given a Banach space $E$, its dual space will be denoted by $E^{\prime}$. Similarly, if $T: E \rightarrow F$ is a bounded linear operator between Banach spaces $E$ and $F$, its dual map will be denoted $T^{\prime}: F^{\prime} \rightarrow E^{\prime}$. Finally, if $p \in(1, \infty)$, we will 
write $p^{\prime}$ for its conjugate Hölder exponent, which satisfies $\frac{1}{p}+\frac{1}{p^{\prime}}=1$. (We will reserve the letter $q$ for fiber maps.)

We exclude $p=1$ in our analysis mostly for convenience, because we use duality in many situations. Moreover the theory of $L^{1}$-operator algebras seems not to be as well-behaved as that for $p \in(1, \infty)$, and is in some sense less interesting. For example:

- The reduced $L^{1}$-group algebra of the free group $\mathbb{F}_{2}$ on two generators is not simple, unlike for $p \in(1, \infty)$ (see [33]);

- For a locally compact group $G$, the canonical map $F^{1}(G) \rightarrow F_{\text {red }}^{1}(G)$ is always an isometric isomorphism (even if $G$ is not amenable), unlike for $p \in(1, \infty)$ (see [28] and [12]);

- $L^{1}$-operator algebras are not closed under quotients (see [13]).

We do not know whether the results of this paper carry over to the case $p=1$.

\section{Borel Bundles of BANACH SPACES}

Definition 2.1. Let $X$ be a Borel space. A (standard) Borel Banach bundle over $X$ is a Borel space $\mathcal{Z}$ fibred over $X$ together with

(1) Borel maps $+: \mathcal{Z} * \mathcal{Z} \rightarrow \mathcal{Z},:: \mathbb{C} \times \mathcal{Z} \rightarrow \mathcal{Z}$, and $\|\cdot\|: \mathcal{Z} \rightarrow \mathbb{C}$,

(2) a Borel section $0: X \rightarrow \mathcal{Z}$, and

(3) a sequence $\left(\sigma_{n}\right)_{n \in \omega}$ of Borel sections $\sigma_{n}: X \rightarrow \mathcal{Z}$

such that the following holds:

- $\mathcal{Z}_{x}$ is a reflexive Banach space with zero element $\mathbf{0}_{x}$ for every $x \in X$;

- there is $K>0$ such that, for every $x \in X$, the sequence $\left(\sigma_{n, x}\right)_{n \in \omega}$ is a basis of $\mathcal{Z}_{x}$ with basis constant $K$, and the sequence $\left(\sigma_{n, x}^{\prime}\right)_{n \in \omega}$ is a basis of $\mathcal{Z}_{x}^{\prime}$ with basis constant $K$.

The sequence $\left(\sigma_{n}\right)_{n \in \omega}$ is called a basic sequence for $\mathcal{Z}$, and $K$ is called basis constant for $\left(\sigma_{n}\right)_{n \in \omega}$. We say that $\left(\sigma_{n}\right)_{n \in \omega}$ is an unconditional basic sequence if there exists $K>0$ such that for every $x \in X$, the sequences $\left(\sigma_{n, x}\right)_{n \in \omega}$ and $\left(\sigma_{n, x}^{\prime}\right)_{n \in \omega}$ are unconditional bases of $\mathcal{Z}_{x}$ and $\mathcal{Z}_{x}^{\prime}$ with unconditional basis constant $K$. Finally, we say that $\left(\sigma_{n}\right)_{n \in \omega}$ is a normal basic sequence if $\left\|\sigma_{n, x}\right\|=\left\|\sigma_{n, x}^{\prime}\right\|=1$ for every $n \in \omega$ and $x \in X$.

Example 2.2 (Constant bundles). Let $X$ be a Borel space, let $Z$ be a reflexive Banach space, and set $\mathcal{Z}=X \times Z$. Then $\mathcal{Z}$ with the product Borel structure is naturally a Borel Banach bundle, where each fiber $\mathcal{Z}_{x}$ is isomorphic to $Z$. In the particular case when $Z$ is the field of complex numbers, this is called the trivial bundle over $X$.

Let $q: \mathcal{Z} \rightarrow X$ be a Borel Banach bundle. Then the space of Borel sections of $\mathcal{Z}$ has a natural structure of $B(X)$-module. Accordingly, if $\xi_{1}$ and $\xi_{2}$ are Borel sections of $\mathcal{Z}$ and $f \in B(X)$, we denote by $\xi_{1}+\xi_{2}$ and $f \xi$ the Borel sections given by

$$
\left(\xi_{1}+\xi_{2}\right)_{x}=\left(\xi_{1}\right)_{x}+\left(\xi_{2}\right)_{x} \quad \text { and } \quad(f \xi)_{x}=f(x) \xi_{x}
$$


for every $x$ in $X$.

If $E$ is a Borel subset of $X$, then $q^{-1}(E)$ is canonically a Borel Banach bundle over $E$, called the restriction of $\mathcal{Z}$ to $E$, and denoted by $\left.\mathcal{Z}\right|_{E}$.

Remark 2.3. A Borel Banach bundle where each fiber is a Hilbert space is called a Borel Hilbert bundle. Such bundles (usually called just Hilbert bundles) are the key notion in the study of representation of groupoids on Hilbert spaces; see [40, Appendix F], [26, Section 3.1], and [35, Section 2]. The Gram-Schmidt process shows that a Borel Hilbert bundle $\mathcal{H}$ over $X$ always has a basic sequence $\left(\sigma_{n}\right)_{n \in \omega}$ such that for all $x$ in $X$, the sequence $\left(\sigma_{n, x}\right)_{n \in \omega}$ is an orthonormal basis of $\mathcal{H}_{x}$.

2.1. Canonical Borel structures. Let $X$ be a Borel space, and let $\mathcal{Z}$ be a set (with no Borel structure) fibred over $X$. Assume there are operations

$$
+: \mathcal{Z} * \mathcal{Z} \rightarrow \mathcal{Z} \quad, \quad \cdot: \mathbb{C} \times \mathcal{Z} \rightarrow \mathcal{Z} \text { and }\|\cdot\|: \mathcal{Z} \rightarrow \mathbb{C},
$$

making each fiber a Banach space. In this situation, we will say that $\mathcal{Z}$ is a bundle of Banach spaces over $X$, and will denote it by $\bigsqcup_{x \in X} \mathcal{Z}_{x}$. Let $\mathcal{Z}^{\prime}$ be the set

$$
\mathcal{Z}^{\prime}=\left\{(x, v): x \in X, v \in \mathcal{Z}_{x}^{\prime}\right\} .
$$

Then $\mathcal{Z}^{\prime}$ is also a bundle of Banach spaces over $X$.

Suppose further that there is a sequence $\left(\sigma_{n}\right)_{n \in \omega}$ of Borel sections $\sigma_{n}: X \rightarrow$ $\mathcal{Z}$ such that, for every $x \in X$, the sequence $\left(\sigma_{n, x}\right)_{n \in \omega}$ is a basis of $\mathcal{Z}_{x}$. For every $x \in X$, denote by $\left(\sigma_{n, x}^{\prime}\right)_{n \in \omega}$ the dual basis of $\mathcal{Z}_{x}^{\prime}$. Assume that for every $m \in \omega$ and every sequence $\left(\alpha_{j}\right)_{j \in m}$ in $\mathbb{Q}(i)^{\oplus m}$, the map $X \rightarrow \mathbb{R}$ given by $x \mapsto\left\|\sum_{j \in m} \alpha_{j} \sigma_{j, x}\right\|$ is Borel. Set

$$
Z=\left\{\left(x,\left(\alpha_{n}\right)_{n \in \omega}\right) \in X \times \mathbb{C}^{\omega}: \sup _{n \in \omega}\left\|\sum_{j \in n} \alpha_{j} \sigma_{j, x}\right\|<\infty\right\} .
$$

We claim that $Z$ is a Borel subset of $X \times \mathbb{C}^{\omega}$. To see this, note that a pair $\left(x,\left(\alpha_{n}\right)_{n \in \omega}\right)$ in $X \times \mathbb{C}^{\omega}$ belongs to $Z$ if and only if there is $N \in \omega$ such that for every $m, k \in \omega$ there is $\left(\beta_{j}\right)_{j \in m}$ in $\mathbb{Q}(i)^{\oplus m}$ such that

$$
\max _{j \in n}\left|\alpha_{j}-\beta_{j}\right| \leq \frac{1}{2^{k}} \quad \text { and } \quad\left\|\sum_{j \in m} \beta_{j} \sigma_{j, x}\right\|<\infty .
$$

Since the map $x \mapsto\left\|\sum_{j \in m} \beta_{j} \sigma_{j, x}\right\|$ is Borel, this proves the claim.

The assignment $\left(x,\left(\alpha_{n}\right)_{n \in \omega}\right) \mapsto \sum_{n \in \omega} \alpha_{n} \sigma_{n, x}$ induces a bijection $Z \rightarrow \mathcal{Z}$ since, for every $x \in X$, the sequence $\left(\sigma_{n, x}\right)_{n \in \omega}$ is a boundedly complete basis of $\mathcal{Z}_{x}$. This bijection induces a standard Borel structure on $\mathcal{Z}$, and 
it is not difficult to verify that such Borel structure turns $\mathcal{Z}$ into a Borel Banach bundle.

A similar argument shows that the set

$$
Z^{\prime}=\left\{\left(x,\left(\alpha_{n}\right)_{n \in \omega}\right) \in X \times \mathbb{C}^{\omega}: \sup _{n \in \omega}\left\|\sum_{j \in n} \alpha_{j} \sigma_{j, x}^{\prime}\right\|<\infty\right\}
$$

is Borel, and that the map from $Z^{\prime}$ to $\mathcal{Z}^{\prime}$ given by $\left(x,\left(\alpha_{n}\right)_{n \in \omega}\right) \mapsto \sum_{n \in \omega} \alpha_{n} \sigma_{n, x}^{\prime}$ is a bijection. This induces a standard Borel structure on $\mathcal{Z}^{\prime}$ that makes $\mathcal{Z}^{\prime}$ a Borel Banach bundle.

It follows from the definition of the Borel structures on $\mathcal{Z}$ and $\mathcal{Z}^{\prime}$, that the canonical pairing $\mathcal{Z} * \mathcal{Z}^{\prime} \rightarrow \mathbb{C}$ is Borel. In fact, for $\left(x,\left(\alpha_{n}\right)_{n \in \omega}\right) \in Z$ and $\left(x,\left(\beta_{n}\right)_{n \in \omega}\right) \in Z^{\prime}$, we have

$$
\left\langle\sum_{n \in \omega} \alpha_{n} \sigma_{n, x}, \sum_{m \in \omega} \beta_{m} \sigma_{m, x}^{\prime}\right\rangle=\sum_{n \in \omega} \alpha_{n} \bar{\beta}_{n} .
$$

The standard Borel structures on $\mathcal{Z}$ and $\mathcal{Z}^{\prime}$ here described will be referred to as the canonical Borel structures associated with the sequence $\left(\sigma_{n}\right)_{n \in \omega}$ of Borel sections $X \rightarrow \mathcal{Z}$. By [17, Theorem 14.12], these can be equivalently described as the Borel structures generated by the sequence of functionals on $\mathcal{Z}$ and $\mathcal{Z}^{\prime}$ given by

$$
z \mapsto\left\langle z, \sigma_{n, q(z)}^{\prime}\right\rangle \quad \text { and } \quad w \mapsto\left\langle\sigma_{n, q(w)}, w\right\rangle
$$

for $n \in \omega$.

As a consequence of the previous discussion, we conclude that if $\mathcal{Z}$ is a Borel Banach bundle, then the Borel structure on $\mathcal{Z}$ is generated by the sequence of maps $\mathcal{Z} \rightarrow \mathbb{C}$ given by $z \mapsto\left\langle z, \sigma_{n, q(z)}^{\prime}\right\rangle$ for $n$ in $\omega$. Moreover, the dual bundle $\mathcal{Z}^{\prime}$ has a unique Borel Banach bundle structure making the canonical pairing Borel. In the following, whenever $\mathcal{Z}$ is a Borel Banach bundle, we will always consider $\mathcal{Z}^{\prime}$ as a Borel Banach bundle endowed with such canonical Borel structure.

The following criterion to endow a Banach bundle with a Borel structure is an immediate consequence of the observations contained in this subsection.

Lemma 2.4. Let $\left(Z_{k}\right)_{k \in \omega}$ be a sequence of reflexive Banach spaces. For every $k \in \omega$, let $\left(b_{n, k}\right)_{n \in \omega}$ be a basis of $Z_{k}$ with dual basis $\left(b_{n, k}^{\prime}\right)_{n \in \omega}$, and suppose that both $\left(b_{n, k}\right)_{n \in \omega}$ and $\left(b_{n, k}^{\prime}\right)_{n \in \omega}$ have basis constant $K$ independent of $k$. Let $\mathcal{Z}$ be a bundle of Banach spaces over $X$, and assume there exist a Borel partition $\left(X_{k}\right)_{k \in \omega}$ of $X$, and isometric isomorphisms $\psi_{x}: Z_{k} \rightarrow \mathcal{Z}_{x}$ and $\psi_{x}^{\prime}: Z_{k}^{\prime} \rightarrow \mathcal{Z}_{x}^{\prime}$ for $k \in \omega$ and $x \in X_{k}$. For $k, n \in \omega$ and $x \in X_{k}$, set $\sigma_{n, x}=\psi_{x}\left(b_{n, k}\right)$ and $\sigma_{n, x}^{\prime}=\psi_{x}^{\prime}\left(b_{n, k}^{\prime}\right)$. There are unique Borel Banach bundle structures on $\mathcal{Z}$ and $\mathcal{Z}^{\prime}$ such that $\left(\sigma_{n}\right)_{n \in \omega}$ and $\left(\sigma_{n}^{\prime}\right)_{n \in \omega}$ are basic sequences, and such that the canonical pairing between $\mathcal{Z}$ and $\mathcal{Z}^{\prime}$ is Borel. 
2.2. Banach space valued $L^{p}$-spaces. For the remainder of this section, we fix a Borel Banach bundle $q: \mathcal{Z} \rightarrow X$ over the standard Borel space $X$, a basic sequence $\left(\sigma_{n}\right)_{n \in \omega}$ of $\mathcal{Z}$ with basis constant $K$, a $\sigma$-finite Borel measure $\mu$ on $X$, and a Hölder exponent $p \in(1, \infty)$.

Definition 2.5. Denote by $\mathcal{L}^{p}(X, \mu, \mathcal{Z})$ the space of Borel sections $\xi: X \rightarrow$ $\mathcal{Z}$ such that

$$
N_{p}(\xi)^{p}=\int\left\|\xi_{x}\right\|^{p} d \mu(x)<\infty .
$$

It follows from the Minkowski inequality that $\mathcal{L}^{p}(X, \mu, \mathcal{Z})$ is a seminormed complex vector space. We denote by $L^{p}(X, \mu, \mathcal{Z})$ the normed space obtained as a quotient of the seminormed space $\left(\mathcal{L}^{p}(X, \mu, \mathcal{Z}), N_{p}\right)$.

When $\mathcal{Z}$ is the trivial bundle over $X$, then $L^{p}(X, \mu, \mathcal{Z})$ coincides with the Banach space $L^{p}(X, \mu)$. Consistently, we will abbreviate $\mathcal{L}^{p}(X, \mu, \mathcal{Z})$ and $L^{p}(X, \mu, \mathcal{Z})$ to $\mathcal{L}^{p}(\mu, \mathcal{Z})$ and $L^{p}(\mu, \mathcal{Z})$, respectively.

Theorem 2.6. The normed vector space $L^{p}(\mu, \mathcal{Z})$ is a Banach space.

Proof. We need to show that the norm on $L^{p}(\mu, \mathcal{Z})$ is complete. In order to show this, it is enough to prove that if $\left(\xi_{n}\right)_{n \in \omega}$ is a sequence in $\mathcal{L}^{p}(\mu, \mathcal{Z})$ such that $\sum_{n \in \omega} N_{p}\left(\xi_{n}\right)<\infty$, then there is $\xi \in \mathcal{L}^{p}(\mu, \mathcal{Z})$ such that

$$
\lim _{m \rightarrow \infty} N_{p}\left(\xi-\sum_{n \in m} \xi_{n}\right)=0 .
$$

Let $\left(\xi_{n}\right)_{n \in \omega}$ be such a sequence. We use Fatou's Lemma at the second step and Jensen's inequality at the fourth to obtain

$$
\begin{aligned}
\int\left(\sum_{n \in \omega}\left\|\xi_{n, x}\right\|\right)^{p} d \mu(x) & =\int \lim _{n \rightarrow \infty}\left(\sum_{j \in n}\left\|\xi_{j, x}\right\|\right)^{p} d \mu(x) \\
& \leq \liminf _{n \rightarrow \infty} \int\left(\sum_{j \in n}\left\|\xi_{j, x}\right\|\right)^{p} d \mu(x) \\
& =\liminf _{n \rightarrow \infty} N_{p}\left(\sum_{j \in n} \xi_{j}\right)^{p} \\
& \leq \liminf _{n \rightarrow \infty}\left(\sum_{j \in n} N_{p}\left(\xi_{j}\right)\right)^{p} \\
& =\left(\sum_{n \in \omega} N_{p}\left(\xi_{n}\right)\right)^{p}<\infty
\end{aligned}
$$


Therefore, the Borel set

$$
F=\left\{x \in X: \sum_{n \in \omega}\left\|\xi_{n, x}\right\|<\infty\right\}
$$

is $\mu$-conull. Using that $\mathcal{Z}_{x}$ is a Banach space for all $x \in X$, we conclude that the sequence $\left(\sum_{j \in n} \xi_{j, x}\right)_{n \in \omega}$ converges to an element $\xi_{x}$ of $\mathcal{Z}_{x}$ for all $x \in F$. Set $\xi_{x}=\mathbf{0}_{x}$ for $x \in X \backslash F$. The resulting map $\xi: X \rightarrow \mathcal{Z}$ is a section, and we claim that it is Borel. To see this, it is enough to observe that the identity

$$
\left\langle\xi_{x}, \sigma_{k, x}^{\prime}\right\rangle= \begin{cases}\sum_{n \in \omega}\left\langle\xi_{n, x}, \sigma_{k}^{\prime}(x)\right\rangle & \text { if } x \in F \\ 0 & \text { otherwise }\end{cases}
$$

implies that the assignment $x \mapsto\left\langle\xi_{x}, \sigma_{k, x}^{\prime}\right\rangle$ is Borel. The claim now follows.

Finally,

$$
N_{p}\left(\xi-\sum_{j \in n} \xi_{n}\right)=\int\left\|\sum_{j \geq n} \xi_{j, x}\right\|^{p} d \mu(x) \leq\left(\sum_{j \geq n} N_{p}\left(\xi_{j, x}\right)\right)^{p},
$$

and since $\lim _{n \rightarrow \infty}\left(\sum_{j \geq n} N_{p}\left(\xi_{j, x}\right)\right)^{p}=0$, the proof is complete.

As it is customary, we will identify an element of $\mathcal{L}^{p}(\mu, \mathcal{Z})$ with its image in the quotient $L^{p}(\mu, \mathcal{Z})$. We will also write $\|\cdot\|_{p}$, or just $\|\cdot\|$ if no confusion is likely to arise, for the norm on $L^{p}(\mu, \mathcal{Z})$ induced by $N_{p}$.

Lemma 2.7. Suppose that $\left(\xi_{n}\right)_{n \in \omega}$ is a sequence in $L^{p}(\mu, \mathcal{Z})$ converging in norm to an element $\xi$ in $L^{p}(\mu, \mathcal{Z})$. Then there are a $\mu$-conull Borel subset $X_{0}$ of $X$, and a subsequence $\left(\xi_{n_{k}}\right)_{k \in \omega}$ such that $\lim _{k \rightarrow \infty}\left\|\xi_{n_{k}, x}-\xi_{x}\right\|=0$ for every $x \in X_{0}$.

Proof. Given $\varepsilon>0$ and $n \in \omega$, set

$$
F_{n, \varepsilon}=\left\{x \in X:\left\|\xi_{n, x}-\xi_{x}\right\| \geq \varepsilon\right\} .
$$

Then $\lim _{n \rightarrow \infty} \mu\left(F_{n, \varepsilon}\right)=0$ by Chebyshev's inequality. Find an increasing sequence $\left(n_{k}\right)_{k \in \omega}$ in $\omega$ such that $\mu\left(F_{m, 2^{-k}}\right) \leq 2^{-k}$ for every $m \geq n_{k}$, and set

$$
F=\bigcap_{k \in \omega} \bigcup_{m \geq n_{k}} F_{m, 2^{-k}} .
$$

Then $\mu(F)=0$ and moreover $\lim _{k \rightarrow \infty}\left\|\xi_{n_{k}, x}-\xi_{x}\right\|=0$ for all $x \in X \backslash F$. This concludes the proof.

Proposition 2.8. Let $\xi \in L^{p}(\mu, \mathcal{Z})$. Then:

(1) The function $\left\langle\xi, \sigma_{n}^{\prime}\right\rangle: X \rightarrow \mathbb{R}$ defined by $x \mapsto\left\langle\xi_{x}, \sigma_{n, x}^{\prime}\right\rangle$ belongs to $\mathcal{L}^{p}(\mu)$ 
(2) The sequence $\left(\sum_{k \in n}\left\langle\xi, \sigma_{k}^{\prime}\right\rangle \sigma_{k}\right)_{n \in \omega}$ converges to $\xi$.

Proof. (1). The function $\left\langle\xi, \sigma_{n}^{\prime}\right\rangle$ is Borel because the canonical pairing map is Borel. Moreover, the estimate

$$
\int\left|\left\langle\xi_{x}, \sigma_{n, x}^{\prime}\right\rangle\right|^{p} d \mu(x) \leq(2 K)^{p} \int\left\|\xi_{x}\right\|^{p} d \mu(x)=(2 K\|\xi\|)^{p}
$$

shows that $\left\langle\xi, \sigma_{n}^{\prime}\right\rangle$ belongs to $\mathcal{L}^{p}(\mu)$.

(2). For every $x \in X$, and using that $K$ is a basis constant for $\left(\sigma_{n, x}\right)_{n \in \omega}$, we have

$$
\left\|\sum_{k \in n}\left\langle\xi_{x}, \sigma_{k, x}^{\prime}\right\rangle \sigma_{k, x}\right\| \leq K\left\|\xi_{x}\right\| .
$$

Given $\varepsilon>0$ and $n \in \omega$, define the Borel set

$$
F_{n, \varepsilon}=\left\{x \in X:\left\|\sum_{k \in n}\left\langle\xi_{x}, \sigma_{k, x}^{\prime}\right\rangle \sigma_{k, x}-\xi_{x}\right\| \leq \varepsilon\right\} .
$$

Then $\bigcup_{n \in \omega} F_{n, \varepsilon}=X$. By the dominated convergence theorem, there is $n_{0} \in \omega$ such that

$$
\int_{X \backslash F_{n_{0}, \varepsilon}}\left\|\xi_{x}\right\|^{p} d \mu(x)<\varepsilon
$$

Thus, for $n \geq n_{0}$, we have

$$
\begin{aligned}
\left\|\sum_{k \in n}\left\langle\xi, \sigma_{k}^{\prime}\right\rangle \sigma_{k}-\xi\right\|_{p}^{p} & =\int\left\|\sum_{k \in n}\left\langle\xi_{x}, \sigma_{k, x}^{\prime}\right\rangle \sigma_{k, x}-\xi_{x}\right\|^{p} d \mu(x) \\
& \leq \mu\left(F_{n, \varepsilon}\right) \varepsilon+(K+1)^{p} \int_{X \backslash F_{n, \varepsilon}}\left\|\xi_{x}\right\|^{p} d \mu(x) \\
& \leq\left((K+1)^{p}+1\right) \varepsilon .
\end{aligned}
$$

This shows that the sequence $\left(\sum_{k \in n}\left\langle\xi, \sigma_{k}^{\prime}\right\rangle \sigma_{k}\right)_{n \in \omega}$ converges to $\xi$.

In view of Proposition 2.8, the sequence $\left(\sigma_{n}\right)_{n \in \omega}$ can be thought as a basis of $L^{p}(\mu, \mathcal{Z})$ over $L^{p}(\mu)$. In particular, Proposition 2.8 implies that $L^{p}(\mu, \mathcal{Z})$ is a separable Banach space. It is not difficult to verify that, if $\left(\sigma_{n}\right)_{n \in \omega}$ is an unconditional basic sequence for $\mathcal{Z}$, then the series $\sum_{k \in \omega}\left\langle\xi, \sigma_{k}^{\prime}\right\rangle \sigma_{k}$ converges unconditionally to $\xi$ for every $\xi \in L^{p}(\mu, \mathcal{Z})$.

Proposition 2.9. Let $\left(f_{n}\right)_{n \in \omega}$ be a sequence in $L^{p}(\mu)$ such that

$$
\sup _{n \in \omega}\left\|\sum_{k \in n} f_{k} \sigma_{k}\right\|_{p}
$$


is finite. Then the sequence

$$
\left(\sum_{k \in n} f_{k} \sigma_{k}\right)_{n \in \omega}
$$

of partial sums converges in $L^{p}(\mu, \mathcal{Z})$.

Proof. Set $M=\sup _{n \in \omega}\left\|\sum_{k \in n} f_{k} \sigma_{k}\right\|^{p}$ and fix $N \in \omega$. Given $n \in \omega$, define

$$
F_{n}^{N}=\left\{x \in X:\left\|\sum_{k \in n} f_{k}(x) \sigma_{k, x}\right\| \leq 2 N M\right\} .
$$

Then $F_{n}^{N}$ is Borel and $\mu\left(F_{n}^{N}\right) \geq 1-\frac{1}{N}$. Set

$$
F^{N}=\bigcap_{n \in \omega} \bigcup_{k \geq n} F_{k}^{N}
$$

Then $\mu\left(F^{N}\right) \geq 1-\frac{1}{N}$. Since $\left(\sigma_{n, x}\right)_{n \in \omega}$ is a basis for $\mathcal{Z}_{x}$ with basis constant $K$, we have

$$
\sup _{m \in \omega}\left\|\sum_{k \in m} f_{k}(x) \sigma_{k, x}\right\| \leq 2 N M K<\infty
$$

for every $x \in F^{N}$. We conclude that the Borel set

$$
F=\left\{x \in X: \sup _{m \in \omega}\left\|\sum_{k \in m} f_{k}(x) \sigma_{k, x}\right\|<\infty\right\}
$$

is $\mu$-conull. Given $x \in F$, and since $\left(\sigma_{n, x}\right)_{n \in \omega}$ is a boundedly complete basis of $\mathcal{Z}_{x}$, the series $\sum_{n \in \omega} f_{n}(x) \sigma_{n, x}$ converges to an element $\xi_{x}$ of $\mathcal{Z}_{x}$. Defining $\xi_{x}=\mathbf{0}_{x}$ for $x \in X \backslash F$, one obtains a Borel section $\xi: X \rightarrow \mathcal{Z}$. Moreover,

$$
\int\left\|\xi_{x}\right\|^{p} d \mu(x) \leq \sup _{n \in \omega} \int\left\|\sum_{k \in n} f_{k}(x) \sigma_{k, x}\right\|^{p} d \mu(x) \leq M,
$$

and hence $\xi$ belongs to $L^{p}(\mu, \mathcal{Z})$. It follows from Proposition 2.8 that $\xi$ is the limit in $L^{p}(\mu, \mathcal{Z})$ of $\left(\sum_{k \in n} f_{k} \sigma_{k}\right)_{n \in \omega}$.

2.3. Pairing. In this subsection, we show that there is a natural pairing between $L^{p}(\mu, \mathcal{Z})$ and $L^{p^{\prime}}\left(\mu, \mathcal{Z}^{\prime}\right)$, under which we may identify $L^{p}(\mu, \mathcal{Z})^{\prime}$ with $L^{p^{\prime}}\left(\mu, \mathcal{Z}^{\prime}\right)$. We describe this pairing first.

Define a map

$$
\langle\cdot, \cdot\rangle: L^{p}(\mu, \mathcal{Z}) \times L^{p^{\prime}}\left(\mu, \mathcal{Z}^{\prime}\right) \rightarrow \mathbb{C} \quad \text { by } \quad\langle\xi, \eta\rangle=\int\left\langle\xi_{x}, \eta_{x}\right\rangle d \mu(x)
$$

for all $\xi \in L^{p}(\mu, \mathcal{Z})$ and all $\eta \in L^{p^{\prime}}\left(\mu, \mathcal{Z}^{\prime}\right)$. To show that this map is welldefined, we must check that the assignment $x \mapsto\left\langle\xi_{x}, \eta_{x}\right\rangle$ is integrable. For 
this, assuming without loss of generality that $\|\xi\|_{p}=\|\eta\|_{p^{\prime}}=1$, we use Young's inequality at the second step to get

$$
\begin{aligned}
\int\left|\left\langle\xi_{x}, \eta_{x}\right\rangle\right| d \mu(x) & \leq \int\left\|\xi_{x}\right\|\left\|\eta_{x}\right\| d \mu(x) \\
& \leq \frac{1}{p} \int\left\|\xi_{x}\right\|^{p} d \mu(x)+\frac{1}{p^{\prime}} \int\left\|\eta_{x}\right\|^{p^{\prime}} d \mu(x)=1 .
\end{aligned}
$$

Theorem 2.10. The function from $L^{p^{\prime}}\left(\mu, \mathcal{Z}^{\prime}\right)$ to $L^{p}(\mu, \mathcal{Z})^{\prime}$ given by

$$
\eta \mapsto\langle\cdot, \eta\rangle=\int\left\langle{ }_{x}, \eta_{x}\right\rangle d \mu(x)
$$

is an isometric isomorphism.

Proof. We first show that such a function is isometric. Fix $\varepsilon>0$ and fix $\eta \in$ $L^{p^{\prime}}\left(\mu, \mathcal{Z}^{\prime}\right)$ with $\|\eta\|_{p^{\prime}}=1$. Set $\mathcal{Z}_{0}=\left\{z \in \mathcal{Z}:\left(\left\langle z, \sigma_{n, q(z)}\right\rangle\right)_{n \in \omega} \in \mathbb{Q}(i)^{\oplus \omega}\right\}$ and

$$
F=\left\{z \in \mathcal{Z}_{0}:\|z\| \leq 1 \text { and }(1-\varepsilon)\left\|\eta_{q(z)}\right\| \leq\left|\left\langle z, \eta_{q(z)}\right\rangle\right|\right\} .
$$

Then $q(F)=X$, and the fiber map $q$ is countable-to-one on $F$. By [17, Theorem 18.10], there is a Borel section $\tau: X \rightarrow \mathcal{Z}$ such that $\tau_{x} \in F$ for every $x \in X$. Define a Borel section $\xi: X \rightarrow \mathcal{Z}$ by $\xi_{x}=\left\|\eta_{x}\right\|^{p^{\prime}-1} \tau_{x}$ for $x \in X$. Then

$$
\|\xi\|^{p}=\int\left\|\xi_{x}\right\|^{p} d \mu(x) \leq \int\left\|\eta_{x}\right\|^{p^{\prime}} d \mu(x)=1,
$$

and thus $\xi$ belongs to $L^{p}(\mu, \mathcal{Z})$. Finally,

$$
\int\left|\left\langle\xi_{x}, \eta_{x}\right\rangle\right| d \mu(x) \geq(1-\varepsilon) \int\left\|\eta_{x}\right\|^{p^{\prime}} d \mu(x)=1-\varepsilon
$$

and thus $\|\langle\cdot, \eta\rangle\| \geq\|\eta\|$. Since the opposite inequality is immediate, we conclude that the function $\eta \mapsto\langle\cdot, \eta\rangle$ is isometric, as desired.

We will now show that such a function is surjective. Let $\Phi \in L^{p}(\mu, \mathcal{Z})^{\prime}$ be given. For every $n \in \omega$, define the Borel measure $\lambda_{n}$ on $X$ by $\lambda_{n}(E)=$ $\Phi\left(\chi_{E} \sigma_{n}\right)$ for $E \subseteq X$. Then $\lambda_{n}$ is absolutely continuous with respect to $\mu$. Denote by $g_{n}=\frac{d \lambda_{n}}{d \mu}$ the corresponding Radon-Nikodym derivative, which belongs to $L^{1}(\mu)$. Then

$$
\Phi\left(\chi_{E} \sigma_{n}\right)=\int \chi_{E} g_{n} d \mu
$$

for all Borel subsets $E \subseteq X$ and all $n \in \omega$. By continuity, we have $\Phi\left(f \sigma_{n}\right)=$ $\int f g_{n} d \mu$ for every bounded Borel function $f$ on $X$.

Fix $n \in \omega$. We claim that $g_{n}$ belongs to $L^{p^{\prime}}(\mu)$. Let $h$ be a Borel function on $X$ of modulus one such that $h g_{n}=\left|g_{n}\right|$. Given $k$ in $\omega$, set $E_{k}=\left\{x:\left|g_{n}(x)\right| \leq k\right\}$ and define a bounded Borel function $h_{k}: X \rightarrow \mathbb{C}$ by

$$
h_{k}=\chi_{E_{k}} h\left|g_{n}\right|^{p^{\prime}-1} .
$$


It is readily checked that $\left|h_{k}\right|^{p}$ coincides with $\left|g_{n}\right|^{p^{\prime}}$ on $E_{k}$. We use this at the last step to get

$$
\begin{aligned}
\|\Phi\|\left(\int_{E_{k}}\left|g_{n}\right|^{p^{\prime}} d \mu\right)^{\frac{1}{p}} & =\|\Phi\|\left(\int\left|h_{k}\right|^{p} d \mu\right)^{\frac{1}{p}} \geq \Phi\left(h_{k} \sigma_{n}\right) \\
& =\int h_{k} g_{n} d \mu=\int_{E_{k}}\left|g_{n}\right|^{p^{\prime}} d \mu(x) .
\end{aligned}
$$

It follows that $\left(\int_{E_{k}}\left|g_{n}\right|^{p^{\prime}} d \mu(x)\right)^{\frac{1}{p^{\prime}}} \leq\|\Phi\|$. Since $k$ is arbitrary, an application of the monotone convergence theorem yields $\left\|g_{n}\right\|_{p^{\prime}} \leq\|\Phi\|$, and hence $g_{n} \in L^{p^{\prime}}(\mu)$. The claim is proved. Let $K$ be a basis constant for $\left(\sigma_{n}\right)_{n \in \omega}$. We claim that

$$
\sup _{n \in \omega}\left\|\sum_{j \in n} g_{j} \sigma_{j}^{\prime}\right\|_{p^{\prime}} \leq K\|\Phi\| .
$$

Fix $\xi \in L^{p}(\mu, \mathcal{Z})$, and write $\xi=\sum_{n \in \omega} f_{n} \sigma_{n}$ as in Proposition 2.8. Then

$$
\begin{aligned}
\left|\int\left\langle\xi, \sum_{j \in n} g_{j} \sigma_{j}^{\prime}\right\rangle d \mu\right| & =\left|\sum_{j \in n} \int f_{j} g_{j} d \mu\right|=\left|\sum_{j \in n} \Phi\left(f_{j} \sigma_{j}\right)\right| \\
& =\left|\Phi\left(\sum_{j \in n} f_{j} \sigma_{j}\right)\right| \leq\|\Phi\|\left\|\sum_{j \in n} f_{j} \sigma_{j}\right\| \\
& \leq K\|\Phi\|\|\xi\| .
\end{aligned}
$$

This being true for every $\xi \in L^{p}(\mu, \mathcal{Z})$ implies that $\left\|\sum_{j \in n} g_{j} \sigma_{j}^{\prime}\right\| \leq K\|\Phi\|$, and the claim has been proved. We can now conclude from Proposition 2.9 that the sequence $\left(\sum_{j \in n} g_{j} \sigma_{j}^{\prime}\right)_{n \in \omega}$ of partial sums converges to an element $\eta$ in $L^{p^{\prime}}\left(\mu, \mathcal{Z}^{\prime}\right)$. Using Proposition 2.8 it is immediate to verify that

$$
\Phi(\xi)=\int\langle\xi, \eta\rangle d \mu
$$

for every $\xi \in L^{p}(\mu, \mathcal{Z})$. Thus $\Phi=\langle\cdot, \eta\rangle$, and this finishes the proof.

It follows that the Banach space $L^{p}(\mu, \mathcal{Z})$ is reflexive. (Recall that the Banach bundle is assumed to have a basic sequence $\left(\sigma_{n}\right)_{n \in \omega}$, and, in particular, all its fibers are reflexive.)

2.4. Bundles of $L^{p}$-spaces. Consider a Borel probability measure $\mu$ on a standard Borel space $X$. Let $\lambda$ be a Borel probability measure on a standard Borel space $Z$ fibred over $X$ via a fiber map $q$ such that $q_{*}(\lambda)=\mu$. By [17, Exercise 17.35], the measure $\lambda$ admits a disintegration $\left(\lambda_{x}\right)_{x \in X}$ with respect to $\mu$, which is also written as $\lambda=\int \lambda_{x} d \mu(x)$. In other words, 
- there is a Borel assignment $x \mapsto \lambda_{x}$, where $\lambda_{x}$ is a probability measure on $\mathcal{Z}_{x}$, and

- for every bounded Borel function $f: Z \rightarrow \mathbb{C}$, we have

$$
\int f d \lambda=\int\left(\int f d \lambda_{x}\right) d \mu(x) .
$$

Consider the Banach bundle $\mathcal{Z}=\bigsqcup_{x \in X} L^{p}\left(\lambda_{x}\right)$ over $X$, where the fiber $\mathcal{Z}_{x}$ over $x$ is $L^{p}\left(\lambda_{x}\right)$.

Theorem 2.11. There is a canonical Borel Banach bundle structure on $\mathcal{Z}$ such that $L^{p}(\mu, \mathcal{Z})$ is isometrically isomorphic to $L^{p}(\lambda)$.

Proof. Let us assume for simplicity that $\mu$ and $\lambda_{x}$ are atomless for every $x \in X$. In this case, by [14, Theorem 2.2], we can assume without loss of generality that

- $X$ is the unit interval $[0,1]$ and $\mu$ is its Lebesgue measure;

- $Z$ is the unit square $[0,1]^{2}$ and $\lambda$ is its Lebesgue measure;

- $q: Z \rightarrow X$ is the projection onto the first coordinate; and

- $\lambda_{x}$ is the Lebesgue measure on $\{x\} \times[0,1]$ for every $x \in X$.

Let $\left(h_{n}\right)_{n \in \omega}$ be the Haar system on $[0,1]$ defined as in [7, Chapter 3]. For $n \in \omega$ and $x \in[0,1]$, define $h_{n, x}^{(p)}:[0,1] \rightarrow \mathbb{R}$ by

$$
h_{n, x}^{(p)}(t)=\frac{h_{n}(t)}{\left\|h_{n}\right\|_{p}}
$$

for every $t \in[0,1]$. Then $\left(h_{n, x}^{(p)}\right)_{n \in \omega}$ is a normalized basis of $L^{p}\left(\lambda_{x}\right)$ for every $x \in[0,1]$. It follows from the discussion in Subsection 2.1 that there are unique Borel Banach bundle structures on $\mathcal{Z}$ and $\mathcal{Z}^{\prime}=\bigsqcup_{x \in X} L^{p^{\prime}}\left(\lambda_{x}\right)$ such that $\left(h_{n}^{(p)}\right)_{n \in \omega}$ and $\left(h_{n}^{\left(p^{\prime}\right)}\right)_{n \in \omega}$ are normal basic sequences for $\mathcal{Z}$ and $\mathcal{Z}^{\prime}$, and that the canonical pairing between $\mathcal{Z}$ and $\mathcal{Z}^{\prime}$ is Borel.

We claim that $L^{p}(\mu, \mathcal{Z})$ can be canonically identified with $L^{p}(\lambda)$. Given $f \in L^{p}(\lambda)$, consider the Borel section $s_{f}: X \rightarrow \mathcal{Z}$ defined by $s_{f, x}(t)=f(x, t)$ for $x, t \in[0,1]$. It is clear that $s_{f, x}$ belongs to $L^{p}(\mu, \mathcal{Z})$ and that

$$
\left(\int\left\|s_{f, x}\right\|_{p}^{p} d \mu(x)\right)^{\frac{1}{p}}=\|f\|_{p} .
$$

It follows that the map $f \mapsto s_{f, x}$ induces an isometric linear map $s: L^{p}(\lambda) \rightarrow$ $L^{p}(\mu, \mathcal{Z})$. The fact that $s$ is surjective is a consequence of Proposition 2.8, since the range of $s$ is a closed linear subspace of $L^{p}(\mu, \mathcal{Z})$ that contains $h_{n}^{(p)}$ for every $n \in \omega$.

The case when $\lambda$ and $\mu$ are arbitrary Borel probability measures can be treated similarly, using the classification of disintegration of Borel probability measures given in [14, Theorem 3.2], together with Lemma 2.4. In fact, 
the results of [14] show that the same conclusions hold if $\lambda$ is a Borel $\sigma$-finite measure.

Definition 2.12. Let $X$ be a Borel space, and let $\mu$ be a Borel probability measure on $X$. An $L^{p}$-bundle over $(X, \mu)$ is a Borel Banach bundle $\mathcal{Z}=$ $\bigsqcup_{x \in X} L^{p}\left(\lambda_{x}\right)$ obtained from the disintegration of a $\sigma$-finite Borel measure $\lambda$ on a Borel space $Z$ fibred over $X$, as described in Theorem 2.11,

2.5. Decomposable operators. Let $q_{X}: \mathcal{Z} \rightarrow X$ and $q_{Y}: \mathcal{W} \rightarrow Y$ be standard Borel Banach bundles with basic sequences $\left(\sigma_{n}\right)_{n \in \omega}$ and $\left(\tau_{n}\right)_{n \in \omega}$, respectively, and let $\phi: X \rightarrow Y$ be a Borel isomorphism.

Definition 2.13. Let $B(\mathcal{Z}, \mathcal{W}, \phi)$ be the space of contractive linear maps of the form $T: \mathcal{Z}_{x} \rightarrow \mathcal{W}_{\phi(x)}$ for some $x \in X$. For such a map $T$, we denote the corresponding point $x$ in $X$ by $x_{T}$.

Consider the Borel structure on $B(\mathcal{Z}, \mathcal{W}, \phi)$ generated by the maps $T \mapsto$ $x_{T}$ and $T \mapsto\left\langle T \sigma_{n, x_{T}}, \tau_{m, \phi\left(x_{T}\right)}^{\prime}\right\rangle$ for $n, m \in \omega$. It is not difficult to check that the operator norm and composition of operators are Borel functions on $B(\mathcal{Z}, \mathcal{W}, \phi)$, which make $B(\mathcal{Z}, \mathcal{W}, \phi)$ into a Borel space fibred over $X$.

Lemma 2.14. The Borel space $B(\mathcal{Z}, \mathcal{W}, \phi)$ is standard.

Proof. Let $V$ be set of elements $\left(x,\left(c_{n, m}\right)_{n, m \in \omega}\right)$ in $X \times \mathbb{C}^{\omega \times \omega}$ such that, for some $M \in \omega$ and every $\left(\alpha_{n}\right)_{n \in \omega} \in \mathbb{Q}(i)^{\oplus \omega}$, we have

$$
\sup _{m \in \omega}\left\|\sum_{k \in m}\left(\sum_{n \in \omega} a_{n} c_{n, m}\right) \tau_{\phi(x), m}\right\| \leq M \sup _{n \in \omega}\left\|\sum_{k \in n} \alpha_{k} \sigma_{x, k}\right\| .
$$

Then $V$ is a Borel subset of $X \times \mathbb{C}^{\omega \times \omega}$, and it is therefore a standard Borel space by [17, Corollary 13.4]. The result follows since the function $B(\mathcal{Z}, \mathcal{W}, \phi) \rightarrow X \times \mathbb{C}^{\omega \times \omega}$ given by

$$
T \mapsto\left(x_{T},\left(\left\langle T \sigma_{n, x_{T}}, \tau_{m, \phi\left(x_{T}\right)}^{\prime}\right\rangle\right)_{(n, m) \in \omega \times \omega}\right)
$$

is a Borel isomorphism between $B(\mathcal{Z}, \mathcal{W}, \phi)$ and $V$.

Fix Borel $\sigma$-finite measures $\mu$ on $X$ and $\nu$ on $Y$ with $\phi_{*}(\mu) \sim \nu$.

Proposition 2.15. If $x \mapsto T_{x}$ is a Borel section of $B(\mathcal{Z}, \mathcal{W}, \phi)$ such that, for some $M \geq 0$ and $\mu$-almost every $x \in X$, we have

$$
\left\|T_{x}\right\|^{p} \leq M^{p} \frac{d \phi_{*}(\mu)}{d \nu}(\phi(x)),
$$

then the linear operator $T: L^{p}(\mu, \mathcal{Z}) \rightarrow L^{p}(\nu, \mathcal{W})$ defined by

$$
(T \xi)_{y}=T_{\phi^{-1}(y)} \xi_{\phi^{-1}(y)} .
$$

for all $y \in Y$, is bounded. Moreover, the norm of $T$ is the minimum $M \geq 0$ such that the inequality in (1) holds for $\mu$-almost every $x \in X$. 
Proof. For $\xi$ in $L^{p}(\mu, \mathcal{Z})$, we have

$$
\begin{aligned}
\|T \xi\|^{p} & =\int\left\|(T \xi)_{y}\right\|^{p} d \nu(y)=\int\left\|T_{\phi^{-1}(y)} \xi_{\phi^{-1}(y)}\right\|^{p} d \nu(y) \\
& =\int\left\|T_{\phi^{-1}(y)} \xi_{\phi^{-1}(y)}\right\|^{p} d \nu(y) \leq \int\left\|T_{\phi^{-1}(y)}\right\|^{p}\left\|\xi_{\phi^{-1}(y)}\right\|^{p} d \nu(y) \\
& \leq \int M^{p} \frac{d \phi_{*}(\mu)}{d \nu}(y)\left\|\xi_{\phi^{-1}(y)}\right\|^{p} d \nu(y) \leq M^{p}\|\xi\|^{p} .
\end{aligned}
$$

This shows that $T$ is bounded with norm at most $M$. It remains to show that

$$
\left\|T_{x}\right\|^{p} \leq\|T\|^{p} \frac{d \phi_{*}(\mu)}{d \nu}(\phi(x))
$$

for $\mu$-almost every $x \in X$. For $\alpha \in \mathbb{Q}(i)^{\oplus \omega}$, set

$$
\sigma_{\alpha}=\sum_{n \in \omega} \alpha_{n} \sigma_{n} \in L^{p}(\mu, \mathcal{Z})
$$

and observe that the set $\left\{\sigma_{\alpha, x}: \alpha \in \mathbb{Q}(i)^{\oplus \omega}\right\}$ is dense in $\mathcal{Z}_{x}$ for every $x \in X$. It is therefore enough to show that

$$
\left\|T_{\phi^{-1}(y)} \sigma_{n, \phi^{-1}(y)}\right\|^{p} \leq\|T\|^{p} \frac{d \phi_{*}(\mu)}{d \nu}(y)\left\|\sigma_{n, \phi^{-1}(y)}\right\|^{p}
$$

for every $\alpha \in \mathbb{Q}(i)^{\oplus \omega}$, and for $\nu$-almost every $y \in Y$. In order to show this, let $g: Y \rightarrow \mathbb{C}$ be a bounded Borel function. Then

$$
\begin{aligned}
& \int|g(y)|^{p}\left\|T_{\phi^{-1}(y)} \sigma_{\alpha, \phi^{-1}(y)}\right\|^{p} d \nu \\
& =\left\|T(g \circ \phi) \sigma_{\alpha}\right\|^{p} \\
& \leq\|T\|^{p} \int\left\|g(\phi(x)) \sigma_{\alpha, x}\right\|^{p} d \mu(x) \\
& \leq\|T\|^{p} \int|g(y)|^{p}\left\|\sigma_{\alpha, \phi^{-1}(y)}\right\|^{p} d \phi_{*}(\mu)(x) \\
& =\|T\|^{p} \int|g(y)|^{p}\left\|\sigma_{\alpha, \phi^{-1}(y)}\right\|^{p} \frac{d \phi_{*}(\mu)}{d \nu}(y) d \nu(y) .
\end{aligned}
$$

Since $g$ is arbitrary, this concludes the proof.

Definition 2.16. An operator $T: L^{p}(\mu, \mathcal{Z}) \rightarrow L^{p}(\nu, \mathcal{W})$ obtained from a Borel section $x \mapsto T_{x}$ of $B(\mathcal{Z}, \mathcal{W}, \phi)$ as in Proposition 2.15, is called decomposable with respect to the Borel isomorphism $\phi: X \rightarrow Y$. The Borel section $x \mapsto T_{x}$ corresponding to the decomposable operator $T$ is called the disintegration of $T$ with respect to the Borel isomorphism $\phi: X \rightarrow Y$.

Remark 2.17. It is not difficult to verify that the disintegration of a decomposable operator $T$ is essentially unique, in the sense that if $x \mapsto T_{x}$ and $x \mapsto \widetilde{T}_{x}$ are two Borel sections defining the same decomposable operator, then $T_{x}=\widetilde{T}_{x}$ for $\mu$-almost every $x$ in $X$. 
Given a bounded Borel function $g: Y \rightarrow \mathbb{C}$, denote by $\Delta_{g} \in B\left(L^{p}(\nu, \mathcal{W})\right)$ the corresponding multiplication operator.

We have the following characterization of decomposable operators.

Proposition 2.18. For a bounded map $T: L^{p}(\mu, \mathcal{Z}) \rightarrow L^{p}(\nu, \mathcal{W})$, the following are equivalent:

(1) $T$ is decomposable with respect to $\phi$;

(2) $\Delta_{g} T=T \Delta_{g \circ \phi}$ for every bounded Borel function $g: Y \rightarrow \mathbb{C}$;

(3) There is a countable collection $\mathcal{F}$ of Borel subsets of $Y$ that separates the points of $Y$, such that $\Delta_{\chi_{F}} T=T \Delta_{\chi_{\phi^{-1}[F]}}$ for every $F \in \mathcal{F}$.

Proof. (1) implies (2). Let $x \mapsto T_{x}$ be a Borel section of $B(\mathcal{Z}, \mathcal{W}, \phi)$ such that

$$
(T \xi)_{y}=T_{\phi^{-1}(y)} \xi_{\phi^{-1}(y)}
$$

for every $\xi \in L^{p}(\nu, \mathcal{W})$ and every $y \in Y$. Then

$$
\left(\Delta_{g} T\right)_{y}=g(y) T_{\phi^{-1}(y)} \xi_{\phi^{-1}(y)}=\left(T \Delta_{g \circ \phi} \xi\right)_{y}
$$

for all $y \in Y$.

(2) implies (3). Obvious.

(3) implies (1). For $\left(\alpha_{n}\right)_{n \in \omega} \in \mathbb{Q}(i)^{\oplus \omega}$, set

$$
\sigma_{\alpha}=\sum_{n \in \omega} \alpha_{n} \sigma_{n} \in L^{p}(\mu, \mathcal{Z}) \quad \text { and } \quad \widehat{\sigma}_{\alpha}=T \sigma_{\alpha} \in L^{p}(\nu, \mathcal{W}) .
$$

Using the assumption (3) at the second step, we get

$$
\begin{aligned}
\int_{F}\left\|\widehat{\sigma}_{\alpha, y}\right\|^{p} d \nu(y) & =\left\|\Delta_{\chi_{F}} \widehat{\sigma}_{\alpha}\right\|^{p}=\left\|T \Delta_{\phi^{-1}[F]} \sigma_{\alpha}\right\|^{p} \\
& \leq\|T\|^{p}\left\|\Delta_{\phi^{-1}[F]} \sigma_{\alpha}\right\|^{p} \\
& =\|T\|^{p} \int_{\phi^{-1}[F]}\left\|\sigma_{\alpha, x}\right\|^{p} d \mu(x) \\
& =\|T\|^{p} \int_{F}\left\|\sigma_{\alpha, \phi^{-1}(y)}\right\|^{p} d \phi_{*}(\mu)(y) \\
& =\|T\|^{p} \int_{F}\left\|\sigma_{\alpha, \phi^{-1}(y)}\right\|^{p} \frac{d \phi_{*}(\mu)}{d \nu}(y) d \nu(y)
\end{aligned}
$$

for every $F \in \mathcal{F}$. We conclude that

$$
\left\|\widehat{\sigma}_{\alpha, y}\right\|^{p} \leq\|T\|^{p}\left\|\sigma_{\alpha, \phi^{-1}(y)}\right\|^{p} \frac{d \phi_{*}(\mu)}{d \nu}(y)
$$

for $\nu$-almost every $y \in Y$ and every $\alpha \in \mathbb{Q}(i)^{\oplus \omega}$. It follows that for $\mu$-almost every $x \in X$, the linear map $\sigma_{\alpha, x} \mapsto \widehat{\sigma}_{\alpha, \phi(x)}$ extends to a bounded linear map $T_{x}: \mathcal{Z}_{x} \rightarrow \mathcal{W}_{\phi(x)}$ that satisfies

$$
\left\|T_{x}\right\|^{p} \leq\|T\|^{p}\left\|\sigma_{\alpha, x}\right\|^{p} \frac{d \phi_{*}(\mu)}{d \nu}(\phi(x)) .
$$


Since the assignment $x \mapsto\left\langle T_{x} \sigma_{n, x}, \tau_{m, \phi(x)}\right\rangle$ is Borel, it follows that the map $x \mapsto T_{x}$ defines a Borel section of $B(\mathcal{Z}, \mathcal{W}, \phi)$ satisfying

$$
(T \xi)_{y}=T_{\phi^{-1}(y)} \xi_{\phi^{-1}(y)}
$$

for $\xi \in L^{p}(\mu, \mathcal{Z})$ and $\nu$-almost every $y \in Y$. This concludes the proof.

Definition 2.19. A $\phi$-isomorphism from $\mathcal{Z}$ to $\mathcal{W}$ is a Borel section $x \mapsto T_{x}$ of the bundle $B(\mathcal{Z}, \mathcal{W}, \phi)$ such that $T_{x}$ is a surjective isometry for every $x \in X$.

If $T=\left(T_{x}\right)_{x \in X}$ is a $\phi$-isomorphism from $\mathcal{Z}$ to $\mathcal{W}$, we denote by $T^{-1}$ the $\phi^{-1}$-isomorphism $\left(T_{\phi^{-1}(y)}^{-1}\right)_{y \in Y}$ from $\mathcal{W}$ to $\mathcal{Z}$.

Definition 2.20. If $X=Y$, then $\mathcal{Z}$ and $\mathcal{W}$ are said to be isomorphic if there is an $\operatorname{id}_{X}$-isomorphism from $\mathcal{Z}$ to $\mathcal{W}$. In this case an $\operatorname{id}_{X}$-isomorphism is simply called an isomorphism.

Theorem 2.21. Let $\mathcal{F}$ be a countable collection of Borel subsets of $Y$ that separates the points of $Y$, and let $T: L^{p}(\mu, \mathcal{Z}) \rightarrow L^{p}(\nu, \mathcal{W})$ be an invertible isometry such that

$$
\Delta_{\chi_{F}} T=T \Delta_{\chi_{\phi}-1(F)}
$$

for every $F \in \mathcal{F}$. Then there are a $\mu$-conull subset $X_{0}$ of $X$, a $\nu$-conull subset $Y_{0}$ of $Y$, and a $\phi$-isomorphism $\left.\left.\mathcal{Z}\right|_{X_{0}} \rightarrow \mathcal{W}\right|_{Y_{0}}$ such that $T$ is the decomposable operator associated with the Borel section

$$
x \mapsto\left(\frac{d \phi_{*}(\mu)}{d \nu} \phi(x)\right)^{\frac{1}{p}} T_{x} .
$$

Moreover, if $\widetilde{T}$ is another decomposable operator associated with said Borel section, then $\widetilde{T}_{x}=T_{x}$ for $\mu$-almost every $x \in X$.

Proof. Given $\left(\alpha_{n}\right)_{n \in \omega}$ in $\mathbb{Q}(i)^{\oplus \omega}$, set $\sigma_{\alpha}=\sum_{n \in \omega} \alpha_{n} \sigma_{n} \in L^{p}(\mu, \mathcal{Z})$, and set

$$
\widehat{\sigma}_{\alpha}=\Delta\left(\frac{d \phi_{*}(\mu)}{d \nu}\right)^{-\frac{1}{p}}\left(T \sigma_{\alpha}\right) .
$$

Let $F \in \mathcal{F}$. Then

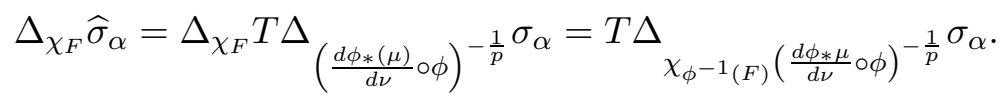


Thus,

$$
\begin{aligned}
\int_{F}\left\|\widehat{\sigma}_{\alpha, y}\right\|^{p} d \nu(y) & =\left\|\Delta_{\chi_{F}} \widehat{\sigma}_{\alpha}\right\|^{p} \\
& =\left\|T \Delta{ }_{\chi_{\phi^{-1}(F)}\left(\frac{d \phi_{*}(\mu)}{d \nu} \circ \phi\right)}{ }^{-\frac{1}{p}} \sigma_{\alpha}\right\|^{p} \\
& \left.=\| \Delta \Delta_{\chi^{-1}(F)}\left(\frac{d \phi_{*}(\mu)}{d \nu} \circ \phi\right)\right)^{-\frac{1}{p}} \sigma_{\alpha} \|^{p} \\
& =\int_{\phi^{-1}[F]}\left(\frac{d \phi_{*}(\mu)}{d \nu} \circ \phi\right)\left\|\sigma_{\alpha, x}\right\|^{p} d \mu(x) \\
& =\int_{F}\left(\frac{d \nu}{d \phi_{*}(\mu)}\right)\left\|\sigma_{\alpha, \phi^{-1}(y)}\right\|^{p} d \phi_{*}(\mu)(y) \\
& =\int_{F}\left\|\sigma_{\alpha, \phi^{-1}(y)}\right\|^{p} d \nu(y) .
\end{aligned}
$$

We conclude that $\left\|\widehat{\sigma}_{\alpha, y}\right\|=\left\|\sigma_{\alpha, \phi^{-1}(y)}\right\|$ for $\nu$-almost every $y \in Y$. Therefore, for $\mu$-almost every $x \in X$, the linear map $\sigma_{\alpha, x} \mapsto \widehat{\sigma}_{\alpha, \phi(x)}$ extends to a linear isometry $T_{x}: \mathcal{Z}_{x} \rightarrow \mathcal{W}_{\phi(x)}$. It can be verified, as in the proof of Proposition 2.18, that $x \mapsto T_{x}$ is a Borel section of $B(\mathcal{Z}, \mathcal{W}, \phi)$. Moreover, it is clear that $T$ is the decomposable operator associated with the section $x \mapsto\left(\left(\frac{d \phi_{*}(\mu)}{d \nu}\right)(\phi(x))\right)^{\frac{1}{p}} T_{x}$

We claim that $T_{x}$ is surjective for $\mu$-almost every $x \in X$. We will do so by constructing a left inverse.

Reasoning as before on $T^{-1}$, one obtains a Borel section $y \mapsto S_{y}$ of $B(\mathcal{W}, \mathcal{Z}, \phi)$ such that $T^{-1}$ is the decomposable operator associated with the Borel section given by $y \mapsto\left(\frac{d \phi^{-1} \nu}{d \mu}\left(\phi^{-1}(y)\right)\right)^{\frac{1}{p}} S_{y}$. Since the disintegration of a decomposable operator is unique, we have

$$
\operatorname{id}_{\mathcal{Z}_{x}}=\left(\frac{d \phi^{-1} \nu}{d \mu}\left(\phi^{-1}(\phi(x))\right)\right)^{\frac{1}{p}} S_{\phi(x)}\left(\left(\frac{d \phi_{*}(\mu)}{d \nu}\right)(\phi(x))\right)^{\frac{1}{p}} T_{x}=S_{\phi(x)} T_{x}
$$

for $\mu$-almost every $x \in X$. This shows that $S_{\phi(x)}$ is the inverse of $T_{x}$ for $\mu$-almost every $x \in X$, and the claim is proved.

The last assertion follows again from uniqueness of the disintegration of a decomposable operator.

\section{BANACH REPRESENTATIONS OF ÉTALE GROUPOIDS}

3.1. Some background notions on groupoids. A groupoid can be defined as a (nonempty) small category where every arrow is invertible. The set of objects of a groupoid $G$ is denoted by $G^{0}$. Identifying an object with its identity arrow, one can regard $G^{0}$ as a subset of $G$. We will denote the 
source and range maps on $G$ by $s, r: G \rightarrow G^{0}$, respectively. The set of pairs of composable arrows

$$
\{(\gamma, \rho) \in G \times G: s(\gamma)=r(\rho)\}
$$

will be denoted, as customary, by $G^{2}$. If $(\gamma, \rho)$ is a pair of composable arrows of $G$, we denote their composition by $\gamma \rho$. If $A$ and $B$ are subsets of $G$, we denote by $A B$ the set

$$
\left\{\gamma \rho:(\gamma, \rho) \in(A \times B) \cap G^{2}\right\} .
$$

Similarly, if $A$ is a subset of $G$ and $\gamma \in G$, then we write $A \gamma$ for $A\{\gamma\}$ and $\gamma A$ for $\{\gamma\} A$. In particular, when $x$ is an object of $G$, then $A x$ denotes the set of elements of $A$ with source $x$, while $x A$ denotes the set of elements of $A$ with range $x$.

A slice of a groupoid $G$ is a subset $A$ of $G$ such that source and range maps are injective on $A$. (Slices are called $G$-sets in [37, 26].) If $U \subseteq G^{0}$, then the set of elements of $G$ with source and range in $U$ is again a groupoid, called the restriction of $G$ to $U$ (or the contraction in [24, 35]), and will be denoted by $G_{\mid U}$.

A locally compact groupoid is a groupoid endowed with a topology having a countable basis of Hausdorff open sets with compact closures, such that

(1) composition and inversion of arrows are continuous maps, and

(2) the set of objects $G^{0}$, as well as $G x$ and $x G$ for every $x \in G^{0}$, are locally compact Haudorff spaces.

It follows that also source and range maps are continuous, since $s(\gamma)=$ $\gamma^{-1} \gamma$ and $r(\gamma)=\gamma \gamma^{-1}$ for all $\gamma \in G$. It should be noted that the topology of a locally compact groupoid might not be (globally) Hausdorff. Examples of non-Hausdorff locally compact groupoids often arise in the applications, such as the holonomy groupoid of a foliation; see [26, Section 2.3]. Locally compact groups are the locally compact groupoids with only one object.

Definition 3.1. An étale groupoid is a locally compact groupoid such that composition of arrows - or, equivalently, the source and range maps - are local homeomorphisms. This in particular implies that $G \gamma$ and $\gamma G$ are countable discrete sets.

Étale groupoids can be regarded as the analog of countable discrete groups. In fact, countable discrete groups are precisely the étale groupoids with only one object.

Definition 3.2. Let $G$ be an étale groupoid. If $U$ is an open Hausdorff subset of $G$, then $C_{c}(U)$ is the space of compactly supported continuous functions on $U$. Recall that $B(G)$ denotes the space of complex-valued Borel functions on $G$. We define $C_{c}(G)$ to be the linear span inside $B(G)$ of the union of all $C_{c}(U)$, where $U$ ranges over the open Hausdorff subsets of $G$. (Equivalently, $U$ ranges over a covering of $G$ consisting of open slices [10, 
Proposition 3.10].) One can define the convolution product and inversion on $C_{c}(G)$ by

$$
(f * g)(\gamma)=\sum_{\rho_{0} \rho_{1}=\gamma} f\left(\rho_{0}\right) g\left(\rho_{1}\right) \quad \text { and } \quad f^{*}(\gamma)=\overline{f\left(\gamma^{-1}\right)}
$$

for $f, g \in C_{c}(G)$. For $f \in C_{c}(G)$, its $I$-norm is given by

$$
\|f\|_{I}=\max \left\{\sup _{x \in G} \sum_{\gamma \in x G}|f(\gamma)|, \sup _{x \in G} \sum_{\gamma \in G x}|f(\gamma)|\right\} \text {. }
$$

These operations turn $C_{c}(G)$ into a normed *-algebra; see [26, Section $2.2]$.

Similarly, one can define the space $B_{c}(G)$ as the linear span inside $B(G)$ of the space of complex-valued bounded functions on $G$ vanishing outside a compact Hausdorff subset of $G$. Convolution product, inversion, and the $I$ norm can be defined exactly in the same way on $B_{c}(G)$ as on $C_{c}(G)$, making $B_{c}(G)$ a normed *algebra; see [26, Section 2.2]. Both $C_{c}(G)$ and $B_{c}(G)$ have a contractive approximate identity.

Remark 3.3. When $G$ is a Hausdorff étale groupoid, then $C_{c}(G)$ as defined above coincides with the space of compactly supported continuous functions on $G$.

Definition 3.4. A representation of $C_{c}(G)$ on a Banach space $Z$ is a homomorphism $\pi: C_{c}(G) \rightarrow B(Z)$. We say that $\pi$ is contractive if it is contractive with respect to the $I$-norm on $C_{c}(G)$.

Let $G$ be an étale groupoid, and let $\mu$ is a Borel probability measure on $G^{0}$. Then $\mu$ induces $\sigma$-finite Borel measures $\nu$ and $\nu^{-1}$ on $G$, which are given by

$$
\nu(A)=\int_{G^{0}}|x A| d \mu(x)
$$

and

$$
\nu^{-1}(A)=\nu\left(A^{-1}\right)=\int_{G^{0}}|A x| d \mu(x)
$$

for every Borel subset $A$ of $G$.

Observe that $\nu$ is the measure obtained integrating the Borel family $\left(c_{x G}\right)_{x \in X}$-where $c_{x G}$ denotes the counting measure on $x G$-with respect to $\mu$. Similarly, $\nu$ is the measure obtained integrating $\left(c_{G x}\right)_{x \in X}$ with respect to $\mu$.

The measure $\mu$ is said to be quasi-invariant if $\nu$ and $\nu^{-1}$ are equivalent, in symbols $\nu \sim \nu^{-1}$. In such case, the Radon-Nikodym derivative $\frac{d \nu}{d \nu^{-1}}$ will be denoted by $D$. Results of Hahn [15] and Ramsay [36, Theorem 3.20] show that one can always choose - as we will do in the following - $D$ to be a Borel homomorphism from $G$ to the multiplicative group of strictly positive real numbers. 
Definition 3.5. An open slice of a groupoid $G$ is an open subset $A$ of $G$ such that source and range maps are injective on $A$. If $A$ is an open slice, then there is a local homeomorphism $\theta_{A}: A^{-1} A \rightarrow A A^{-1}$ given by $\theta_{A}(x)=r(A x)$ for $x \in A^{-1} A$.

Proposition 3.2.2 of [26] shows that, if $A$ is an open slice, then

$$
D(y A)^{-1}=\frac{d\left(\theta_{A}\right)_{*} \mu_{\mid A^{-1} A}}{d \mu_{\mid A A^{-1}}}(y)
$$

for every $y \in G$. Moreover, $\mu$ is quasi-invariant if and only if $d\left(\theta_{A}\right)_{*} \mu_{\mid A^{-1} A} \sim$ $d \mu_{\mid A A^{-1}}$ for every open slice $A$.

It is easy to verify that if $\mu$ and $\widetilde{\mu}$ are equivalent quasi-invariant measures on $G^{0}$, and $\nu$ and $\widetilde{\nu}$ are the corresponding measures on $G$, then $\nu \sim \widetilde{\nu}$ and

$$
\frac{d \nu}{d \widetilde{\nu}}(\gamma)=\frac{d \mu}{d \widetilde{\mu}}(r(\gamma)) \quad \text { and } \quad \frac{d \nu^{-1}}{d(\widetilde{\nu})^{-1}}(\gamma)=\frac{d \mu}{d \widetilde{\mu}}(s(\gamma))
$$

for all $\gamma \in G$. The chain rule then shows that

$$
\frac{d \widetilde{\nu}}{d \widetilde{\nu}^{-1}}(\gamma)=\frac{d \widetilde{\mu}}{d \mu}(r(\gamma)) \frac{d \nu}{d \nu^{-1}}(\gamma) \frac{d \mu}{d \widetilde{\mu}}(s(\gamma))
$$

for all $\gamma \in G$.

Remark 3.6. Étale groupoids can be characterized as those locally compact groupoids whose topology admits a countable basis of open slices.

Closely related to the notion of an étale groupoid is that of an inverse semigroup.

Definition 3.7. An inverse semigroup is a semigroup $S$ such that for every element $s$ of $S$, there exists a unique element $s^{*}$ of $S$ such that $s s^{*} s=s$ and $s^{*} s s^{*}=s^{*}$.

Let $G$ be an étale groupoid, and denote by $\Sigma(G)$ the set of open slices of $G$. The operations

$$
A B=\left\{\gamma \rho:(\gamma, \rho) \in(A \times B) \cap G^{2}\right\} \quad \text { and } \quad A^{-1}=\left\{\gamma^{-1}: \gamma \in A\right\}
$$

turn $\Sigma(G)$ into an inverse semigroup. The set $\Sigma_{c}(G)$ of precompact open slices of $G$ is a subsemigroup of $\Sigma(G)$. Similarly, the set $\Sigma_{\mathcal{K}}(G)$ of compact open slices of $G$ is also a subsemigroup of $\Sigma(G)$.

Definition 3.8. An étale groupoid $G$ is called ample if $\Sigma_{\mathcal{K}}(G)$ is a basis for the topology of $G$. This is equivalent to the assertion that $G^{0}$ has a countable basis of compact open sets. 
3.2. Representations of étale groupoids on Banach bundles. Throughout the rest of this section, we fix an étale groupoid $G$, and a Borel Banach bundle $q: \mathcal{Z} \rightarrow G^{0}$.

Definition 3.9. We define the groupoid of fiber-isometries of $\mathcal{Z}$ by $\operatorname{Iso}(\mathcal{Z})=\left\{(T, x, y): T: \mathcal{Z}_{x} \rightarrow \mathcal{Z}_{y}\right.$ is an invertible isometry, and $\left.x, y \in G^{0}\right\}$. We denote the elements of $\operatorname{Iso}(\mathcal{Z})$ simply by $T: \mathcal{Z}_{x} \rightarrow \mathcal{Z}_{y}$.

The set $\operatorname{Iso}(\mathcal{Z})$ has naturally the structure of groupoid with set of objects $G^{0}$, where the source and range of the fiber-isometry $T: \mathcal{Z}_{x} \rightarrow \mathcal{Z}_{y}$ are $s(T)=$ $x$ and $r(T)=y$, respectively. If $\left(\sigma_{n}\right)_{n \in \omega}$ is a basic sequence for $\mathcal{Z}$, then the Borel structure generated by the maps

$$
T \mapsto\left\langle T \sigma_{n, s(T)}, \sigma_{m, r(T)}\right\rangle
$$

for $n, m \in \omega$, is standard, and makes $\operatorname{Iso}(\mathcal{Z})$ a standard Borel groupoid. This means that Iso $(\mathcal{Z})$ is a groupoid endowed with a standard Borel structure that makes composition and inversion of arrows Borel.

Definition 3.10. Let $\mu$ be a quasi-invariant Borel probability measure on $G^{0}$. A Borel map $T: G \rightarrow \operatorname{Iso}(\mathcal{Z})$ is said to be a $\mu$-almost everywhere homomorphism, if there exists a $\mu$-conull subset $U$ of $G^{0}$ such that the restriction of $T$ to $\left.G\right|_{U}$ is a groupoid homomorphism which is the identity on $U$.

Definition 3.11. A representation of $G$ on $\mathcal{Z}$, is a pair $(\mu, T)$ consisting of an invariant Borel probability measure $\mu$ on $G^{0}$, and a $\mu$-almost everywhere homomorphism $T: G \rightarrow \operatorname{Iso}(\mathcal{Z})$.

If $G$ is a discrete group, then a Borel Banach bundle over $G^{0}$ is just a Banach space $Z$, and a representation of $G$ on $Z$ is a Borel group homomorphism from $G$ to the Polish group $\operatorname{Iso}(Z)$ of invertible isometries of $Z$ endowed with the strong operator topology. (It should be noted here that a Borel group homomorphism from $G$ to $\operatorname{Iso}(Z)$ is automatically continuous by [17, Theorem 9.10].)

Example 3.12 (Dual representation). Let $(\mu, T)$ be a representation of $G$ on $\mathcal{Z}$. The dual representation of $(\mu, T)$ is the representation $\left(\mu, T^{\prime}\right)$ of $G$ on $\mathcal{Z}^{\prime}$ defined by

$$
\left(T^{\prime}\right)_{\gamma}=\left(T_{\gamma^{-1}}\right)^{\prime}: \mathcal{Z}_{s(\gamma)}^{\prime} \rightarrow \mathcal{Z}_{r(\gamma)}^{\prime}
$$

for all $\gamma \in G$.

There is a natural notion of equivalence for representations of $G$ on Banach bundles.

Definition 3.13. Two representations $(\mu, T)$ and $(\widetilde{\mu}, \widetilde{T})$ of $G$ on Borel Banach bundles $\mathcal{Z}$ and $\widetilde{\mathcal{Z}}$ over $G^{0}$, are said to be equivalent, if $\mu \sim \widetilde{\mu}$ and there are a $\mu$-conull (and hence $\widetilde{\mu}$-conull) Borel subset $U$ of $G^{0}$, and an isomorphism $v:\left.\left.\mathcal{Z}\right|_{U} \rightarrow \widetilde{\mathcal{Z}}\right|_{U}$ (see Definition 2.20) such that

$$
\widetilde{T}_{\gamma} v_{s(\gamma)}=v_{r(\gamma)} T_{\gamma}
$$


for every $\left.\gamma \in G\right|_{U}$.

It is clear that two representations are equivalent if and only if their dual representations are equivalent. (Recall our standing assumption that all Borel Banach bundles are endowed with a basic sequence and, in particular, all the fibers are reflexive Banach spaces.)

We now show how to integrate groupoid representations to $L^{p}$-bundles. From now on, we fix a Hölder exponent $p \in(1, \infty)$.

Theorem 3.14. Let $(\mu, T)$ be a representation of $G$ on $\mathcal{Z}$. Then the equation

$$
\left(\pi_{T}(f) \xi\right)_{x}=\sum_{\gamma \in x G} f(\gamma) D(\gamma)^{-\frac{1}{p}} T_{\gamma} \xi_{s(\gamma)}
$$

for $f \in C_{c}(G), \xi \in L^{p}(\mu, \mathcal{Z})$, and $x \in G^{0}$, defines an $I$-norm contractive, nondegenerate representation $\pi_{T}: C_{c}(G) \rightarrow B\left(L^{p}(\mu, \mathcal{Z})\right)$.

Proof. Fix $f \in C_{c}(G), \xi \in L^{p}(\mu, \mathcal{Z})$, and $\eta \in L^{p^{\prime}}\left(\mu, \mathcal{Z}^{\prime}\right)$. We claim that the complex-valued function on $G$ defined by

$$
\gamma \mapsto D^{-\frac{1}{p}}(\gamma) f(\gamma)\left\langle T_{\gamma} \xi_{s(\gamma)}, \eta_{r(\gamma)}\right\rangle
$$

is $\nu$-integrable. This follows from the following estimate where we use Hölder's inequality at the third step:

$$
\begin{aligned}
& \int\left|f(\gamma)\left\langle T_{\gamma} \xi_{s(\gamma)}, \eta_{r(\gamma)}\right\rangle\right| D^{-\frac{1}{p}}(\gamma) d \nu(\gamma) \\
& =\int\left|f(\gamma)\left\langle T_{\gamma} \xi_{s(\gamma)}, \eta_{r(\gamma)}\right\rangle\right| D^{-\frac{1}{p}}(\gamma) d \nu(\gamma) \\
& \leq \int|f(\gamma)|^{\frac{1}{p}}\left\|\xi_{s(\gamma)}\right\| D^{-\frac{1}{p}}(\gamma)|f(\gamma)|^{\frac{1}{p^{\prime}}}\left\|\eta_{r(\gamma)}\right\| d \nu(\gamma) \\
& \leq\left(\int|f(\gamma)|\left\|\xi_{s(\gamma)}\right\|^{p} D^{-1}(\gamma) d \nu(\gamma)\right)^{\frac{1}{p}}\left(\int|f(\gamma)|\left\|\eta_{r(\gamma)}\right\|^{p^{\prime}} d \nu(\gamma)\right)^{\frac{1}{p^{\prime}}} \\
& =\left(\int|f(\gamma)|\left\|\xi_{s(\gamma)}\right\|^{p} d \nu^{-1}(\gamma)\right)^{\frac{1}{p}}\left(\int|f(\gamma)|\left\|\eta_{r(\gamma)}\right\|^{p^{\prime}} d \nu(\gamma)\right)^{\frac{1}{p^{\prime}}} \\
& =\left(\int \sum_{\gamma \in G x}|f(\gamma)|\left\|\xi_{x}\right\|^{p} d \mu(x)\right)^{\frac{1}{p}}\left(\int \sum_{\gamma \in y G} f(y)\left\|\eta_{y}\right\|^{p^{\prime}} d \mu(y)\right)^{\frac{1}{p^{\prime}}} \\
& \leq\|f\|_{I}\|\xi\|\|\eta\| .
\end{aligned}
$$

Therefore, the linear functional $\phi_{T, \xi}(f): L^{p^{\prime}}\left(\mu, \mathcal{Z}^{\prime}\right) \rightarrow \mathbb{C}$ given by

$$
\phi_{T, \xi}(f)(\eta)=\int_{G} f(\gamma) D^{-\frac{1}{p}}(\gamma)\left\langle T_{\gamma} \xi_{s(\gamma)}, \eta_{r(\gamma)}\right\rangle d \nu(\gamma)
$$


for $\eta \in L^{p^{\prime}}\left(\mu, \mathcal{Z}^{\prime}\right)$, satisfies $\left\|\phi_{T, \xi}(f)\right\| \leq\|f\|_{I}\|\xi\|$. By Theorem 2.10, there is a unique element $\pi_{T}(f) \xi$ of $L^{p}(\mu, \mathcal{Z})$ of norm at most $\|f\|_{I}\|\xi\|$, such that

$$
\begin{aligned}
\int\left\langle\left(\pi_{T}(f) \xi\right)_{x}, \eta_{x}\right\rangle d \mu(x) & =\phi_{T, \xi}(f)(\eta) \\
& =\int\left\langle\sum_{\gamma \in x G} f(\gamma) D(\gamma)^{-\frac{1}{p}} T_{\gamma} \xi_{s(\gamma)}, \eta_{x}\right\rangle d \mu(x)
\end{aligned}
$$

for all $\eta \in L^{p^{\prime}}\left(\mu, \mathcal{Z}^{\prime}\right)$. In particular, Equation (2) defines a bounded linear operator $\pi_{T}(f) \in B\left(L^{p}(\mu, \mathcal{Z})\right)$ of norm at most $\|f\|_{I}$.

We claim that $\pi_{T}: C_{c}(G) \rightarrow B\left(L^{p}(\mu, \mathcal{Z})\right)$ is a nondegenerate homomorphism. Given $f$ and $g$ in $C_{c}(G)$, we have

$$
\begin{aligned}
& \left(\pi_{T}(f * g) \xi\right)_{x} \\
& =\sum_{\gamma \in x G}(f * g)(\gamma) D(\gamma)^{-\frac{1}{p}} T_{\gamma} \xi_{s(\gamma)} \\
& =\sum_{\substack { \gamma \in x G \\
\begin{subarray}{c}{\left(\rho_{0}, \rho_{1}\right) \in G^{2} \\
\rho_{0} \rho_{1}=\gamma{ \gamma \in x G \\
\begin{subarray} { c } { ( \rho _ { 0 } , \rho _ { 1 } ) \in G ^ { 2 } \\
\rho _ { 0 } \rho _ { 1 } = \gamma } }\end{subarray}} f\left(\rho_{0}\right) g\left(\rho_{1}\right) D\left(\rho_{0}\right)^{-\frac{1}{p}} D\left(\rho_{1}\right)^{-\frac{1}{p}} T_{\rho_{0}} T_{\rho_{1}} \xi_{s\left(\rho_{1}\right)} \\
& =\sum_{\rho_{0} \in x G} f\left(\rho_{0}\right) D\left(\rho_{0}\right)^{-\frac{1}{p}} T_{\rho_{0}}\left(\sum_{\rho_{1} \in s\left(\rho_{0}\right)} g\left(\rho_{1}\right) D\left(\rho_{1}\right)^{-\frac{1}{p}} T_{\rho_{1}} \xi_{s\left(\rho_{1}\right)}\right) \\
& =\sum_{\rho_{0} \in x G} f\left(\rho_{0}\right) D\left(\rho_{0}\right)^{-\frac{1}{p}} T_{\rho_{0}}\left(\pi_{T}(g) \xi\right)_{s\left(\rho_{0}\right)} \\
& =\left(\pi_{T}(f) \pi_{T}(g) \xi\right)_{x}
\end{aligned}
$$

for $\mu$-almost every $x \in G^{0}$. We conclude that $\pi_{T}$ is multiplicative.

Let us now show that $\pi_{T}$ is nondegenerate. Assume that $\eta \in L^{p^{\prime}}\left(\mu, \mathcal{Z}^{\prime}\right)$ satisfies $\langle\pi(f) \xi, \eta\rangle=0$ for every $f \in C_{c}(G)$ and every $\xi \in L^{p}(\mu, \mathcal{Z})$. We claim that $\eta=0$. Let $\left(\sigma_{n}\right)_{n \in \omega}$ be a basic sequence for $\mathcal{Z}$. For $\alpha \in \mathbb{Q}(i)^{\oplus \omega}$, set $\sigma_{\alpha}=\sum_{n \in \omega} \alpha_{n} \sigma_{n} \in L^{p}(\mu, \mathcal{Z})$. Then $\left\{\sigma_{\alpha, x}: \alpha \in \mathbb{Q}(i)^{\oplus \omega}\right\}$ is a dense subspace of $\mathcal{Z}_{x}$ for every $x \in X$, and therefore $\left\{T_{\gamma} \sigma_{\alpha, x}: \alpha \in \mathbb{Q}(i)^{\oplus \omega}\right\}$ is a dense subspace of $\mathcal{Z}_{r(\gamma)}$ for every $\gamma \in G x$. By assumption,

$$
0=\left\langle\pi(f) \sigma_{\alpha}, \eta\right\rangle=\int D^{-\frac{1}{p}}(\gamma) f(\gamma)\left\langle T_{\gamma} \sigma_{\alpha, s(\gamma)}, \eta_{r(\gamma)}\right\rangle d \nu(\gamma)
$$

for every $f \in C_{c}(G)$. Therefore, $\left\langle T_{\gamma} \sigma_{\alpha, s(\gamma)}, \eta_{r(\gamma)}\right\rangle=0$ for $\nu$-almost every $\gamma \in G$ and for every $\alpha \in \mathbb{Q}(i)^{\oplus \omega}$. This implies that $\eta_{x}=0$ for $\mu$-almost every $x \in G^{0}$, and thus $\eta=0$. This finishes the proof.

Definition 3.15. Let $(\mu, T)$ be a representation of $G$ on $\mathcal{Z}$. We call the representation $\pi_{T}: C_{c}(G) \rightarrow B\left(L^{p}(\mu, \mathcal{Z})\right)$ constructed in the theorem above, the integrated form of $(\mu, T)$. 
Remark 3.16. Given a representation $(\mu, T)$ of $G$ on $\mathcal{Z}$, one can show that there is an $I$-norm contractive nondegenerate representation $\pi_{T}: B_{c}(G) \rightarrow$ $B\left(L^{p}(\mu, \mathcal{Z})\right)$ defined by the same expression as in the statement of Theorem 3.14 .

Definition 3.17. Let $\mu$ be a Borel $\sigma$-finite measure on $G^{0}$ and let $\pi: C_{c}(G) \rightarrow$ $B\left(L^{p}(\mu, \mathcal{Z})\right)$ be an $I$-norm contractive nondegenerate representation. The dual representation of $\pi$ is the $I$-norm contractive nondegenerate representation $\pi^{\prime}: C_{c}(G) \rightarrow B\left(L^{p^{\prime}}\left(\mu, \mathcal{Z}^{\prime}\right)\right)$ given by $\pi^{\prime}(f)=\pi\left(f^{*}\right)^{\prime}$ for all $f \in C_{c}(G)$.

Lemma 3.18. Let $(\mu, T)$ be a representation of $G$ on $\mathcal{Z}$. Then $\pi_{T}(f)^{\prime}=$ $\pi_{T^{\prime}}\left(f^{*}\right)$ for all $f \in C_{c}(G)$.

Proof. The result follows from the following computation, valid for all $\xi \in$ $L^{p}(\mu, \mathcal{Z})$ and all $\eta \in L^{p^{\prime}}\left(\mu, \mathcal{Z}^{\prime}\right)$ :

$$
\begin{aligned}
\left\langle\pi_{T^{\prime}}\left(f^{*}\right) \eta, \xi\right\rangle & =\int f^{\prime}(\gamma) D(\gamma)^{-\frac{1}{p}}\left\langle T_{\gamma^{-1}} \eta_{s(\gamma)}, \xi_{r(\gamma)}\right\rangle d \nu(\gamma) \\
& =\int \overline{f\left(\gamma^{-1}\right)}\left\langle T_{\gamma^{-1}} \eta_{s(\gamma)}, \xi_{r(\gamma)}\right\rangle D(\gamma)^{\frac{1}{p^{\prime}}} d \nu^{-1}(\gamma) \\
& =\int \overline{f(\gamma)}\left\langle T_{\gamma} \eta_{r(\gamma)}, \xi_{s(\gamma)}\right\rangle D(\gamma)^{-\frac{1}{p^{\prime}}} d \nu(\gamma) \\
& =\frac{\int f(\gamma)\left\langle T_{\gamma} \xi_{s(\gamma)}, \eta_{r(\gamma)}\right\rangle d \nu(\gamma)}{\left\langle\pi_{T}(f) \xi, \eta\right\rangle} .
\end{aligned}
$$

Our next goal is to show that two representations of a groupoid on Borel Banach bundles are equivalent if and only if their integrated forms are equivalent.

Theorem 3.19. Let $(\mu, \mathcal{Z})$ and $(\lambda, \mathcal{W})$ be Borel Banach bundles over $G^{0}$, and let $T$ and $S$ be groupoid representations of $G$ on $\mathcal{Z}$ and $\mathcal{W}$, respectively. Then $T$ and $S$ are equivalent if and only if $\pi_{T}$ and $\pi_{S}$ are equivalent.

Proof. Suppose that $T$ and $S$ are equivalent. Thus $\mu \sim \lambda$ and there are a $\mu$-conull Borel subsets $U$ of $G^{0}$ and an isomorphism $v:\left.\mathcal{Z}\right|_{U} \rightarrow \mathcal{W}_{U}$ (see Definition 2.20) such that $v_{r(\gamma)} T_{\gamma}=S_{\gamma} v_{s(\gamma)}$ for every $\left.\gamma \in G\right|_{U}$. Define a linear map $u: L^{p}(\mu, \mathcal{Z}) \rightarrow L^{p}(\lambda, \mathcal{W})$ by

$$
(u \xi)_{x}=\left(\frac{d \mu}{d \lambda}(x)\right)^{\frac{1}{p}} v_{x} \xi_{x}
$$

for $\xi$ in $L^{p}(\mu, \mathcal{Z})$ and $x \in U$. It is easy to check that $u$ is isometric. We claim that $u$ is bijective. For this, it suffices to check that its inverse is given by

$$
\left(u^{-1} \xi\right)_{y}=\left(\frac{d \lambda}{d \mu}(y)\right)^{\frac{1}{p}} v_{y}^{-1} \xi_{y}
$$

for all $\xi \in L^{p}(\lambda, \mathcal{W})$ and all $y \in G^{0}$. We omit the details. 
We claim that $u$ intertwines $\pi_{T}$ and $\pi_{S}$. Once we show this, the "only if" implication will be proved. To prove the claim, fix $\xi$ in $L^{p}(\lambda, \mathcal{W})$ and $x \in G^{0}$. We have

$$
\begin{aligned}
& \left(u \pi_{T}(f) u^{-1} \xi\right)_{x} \\
& =\left(\frac{d \mu}{d \lambda}(x)\right)^{\frac{1}{p}} v_{x}\left(\pi_{T}(f) u^{-1} \xi\right)_{x} \\
& =\left(\frac{d \mu}{d \lambda}(x)\right)^{\frac{1}{p}} v_{x}\left(\sum_{\gamma \in x G} f(\gamma)\left(\frac{d \nu_{\mu}}{d \nu_{\mu}^{-1}}(\gamma)\right)^{-\frac{1}{p}} T_{\gamma}\left(u^{-1} \xi\right)_{s(\gamma)}\right) \\
& =\sum_{\gamma \in x G} f(\gamma)\left(\frac{d \mu}{d \lambda}(x)\right)^{\frac{1}{p}}\left(\frac{d \nu_{\mu}}{d \nu_{\mu}^{-1}}(\gamma)\right)^{-\frac{1}{p}}\left(\frac{d \lambda}{d \mu}(s(\gamma))\right)^{\frac{1}{p}} v_{x} T_{\gamma} v_{s(\gamma)}^{-1} \xi_{s(\gamma)} \\
& =\sum_{\gamma \in x G} f(\gamma)\left(\frac{d \nu_{\lambda}}{d\left(\nu_{\lambda}\right)^{-1}}(\gamma)\right)^{-\frac{1}{p}} S_{\gamma} \xi_{s(\gamma)} \\
& =\left(\pi_{S}(f) \xi\right)_{x}
\end{aligned}
$$

for all $x \in G$, and the claim is proved.

Conversely, assume that $\pi_{T}$ and $\pi_{S}$ are equivalent, and let $u: L^{p}(\mu, \mathcal{Z}) \rightarrow$ $L^{p}(\lambda, \mathcal{W})$ be a surjective isometry such that

$$
u \pi_{T}(f)=\pi_{S}(f) u
$$

for every $f \in C_{c}(G)$. Denote by $\mathcal{I}$ the set of those functions $f$ in $B_{c}(G)$ that satisfy Equation (3). Fix an open subset $U$ of $G$ contained in some compact Hausdorff set. It follows from the dominated convergence theorem that if $\left(f_{n}\right)_{n \in \omega}$ is a uniformly bounded sequence in $\mathcal{I}$ converging to a function $f \in B(U)$, then $f \in \mathcal{I}$. By [26, Lemma 2.2.1], we have $B(U) \subseteq \mathcal{I}$. In particular, if $A$ is an open slice of $G$ contained in some compact Hausdorff set, then $\chi_{A}$ belongs to $\mathcal{I}$.

Let $\mathcal{F}$ be a countable basis for the topology of $G$ consisting of open slices each one of which is contained in some compact Hausdorff set. Apply Theorem 2.21 to find a Borel conull set $X_{0}$ of $G^{0}$ and an isomorphism $v:\left.\left.\mathcal{Z}\right|_{X_{0}} \rightarrow \mathcal{W}\right|_{X_{0}}$ such that

$$
(u \xi)_{x}=\left(\frac{d \mu}{d \lambda}(x)\right)^{\frac{1}{p}} v_{x} \xi_{x}
$$

for all $x \in X_{0}$ and all $\xi \in L^{p}(\mu, \mathcal{Z})$. It is not difficult to verify that $S_{\gamma} v_{s(\gamma)}=$ $v_{r(\gamma)} T_{\gamma}$ for all $\gamma$ in $G$. This finishes the proof.

3.3. Amplification of representations. Given a natural number $n \geq 1$, regard $M_{n}\left(C_{c}(G)\right)$ as a normed *-algebra with respect to the usual matrix 
product and involution, and the $I$-norm

$$
\begin{aligned}
& \left\|\left[f_{i j}\right]_{i, j \in n}\right\|_{I} \\
= & \max \left\{\max _{x \in G^{0}} \max _{j \in n} \sum_{j \in n} \sum_{\gamma \in x G}\left|f_{i j}(\gamma)\right|, \max _{x \in G^{0}} \max _{j \in n} \sum_{j \in n} \sum_{\gamma \in G x}\left|f_{i j}(\gamma)\right|\right\} .
\end{aligned}
$$

Definition 3.20. Let $\mu$ be a $\sigma$-finite Borel measure on $G^{0}$, and let $\pi: C_{c}(G) \rightarrow$ $B\left(L^{p}(\mu, \mathcal{Z})\right)$ be an $I$-norm contractive representation. We define its amplification $\pi^{(n)}: M_{n}\left(C_{c}(G)\right) \rightarrow B\left(\ell^{p}\left(n, L^{p}(\mu, \mathcal{Z})\right)\right)$ by

$$
\pi^{(n)}\left(\left[f_{i j}\right]_{i, j \in n}\right)\left[\xi_{j}\right]_{j \in n}=\left[\sum_{j \in n} \pi\left(f_{i j}\right) \xi_{j}\right]_{i \in n} .
$$

If one starts with a representation $T$ of a groupoid on a Borel Banach bundle, one may take its integrated form, and then its amplification to matrices over $C_{c}(G)$, as in the definition above. The resulting representation $\pi_{T}^{(n)}$ is the integrated form of a representation of an amplified groupoid, which we proceed to describe.

Definition 3.21. Given $n \geq 1$, denote by $G_{n}$ the groupoid $n \times G \times n$ endowed with the product topology, with set of objects $G^{0} \times n$, and operations defined by

$s(i, \gamma, j)=(s(\gamma), j), \quad r(i, \gamma, j)=(r(\gamma), i) \quad$ and $\quad(i, \gamma, j)(j, \rho, k)=(i, \gamma \rho, k)$.

Denote by $\mathcal{Z}^{(n)}$ the Borel Banach bundle over $G^{0} \times n$ such that $\mathcal{Z}_{(x, j)}^{(n)}=\mathcal{Z}_{x}$, with basic sequence $\left(\sigma_{k}^{(n)}\right)_{k \in \omega}$ defined by

$$
\sigma_{k,(x, j)}^{(n)}=\sigma_{k, x}
$$

for $(x, j) \in G^{0} \times n$. Endow $G^{0} \times n$ with the measure $\mu^{(n)}=\mu \times c_{n}$, and define the amplification $T^{(n)}: G_{n} \rightarrow \operatorname{Iso}\left(\mathcal{Z}^{(n)}\right)$ of $T$ by $T_{(i, \gamma, j)}^{(n)}=T_{\gamma}$ for $(i, \gamma, j) \in G_{n}$.

Proposition 3.22. Let $(T, \mu)$ be a representation of $G$ on $\mathcal{Z}$. Given $n \geq 1$, the representations $\pi_{T}^{(n)}$ and $\pi_{T^{(n)}}$ are equivalent.

Proof. Under the canonical identifications

$$
M_{n}\left(C_{c}(G)\right) \cong C_{c}\left(G_{n}\right)
$$

and

$$
\ell^{p}\left(n, L^{p}(\mu, \mathcal{Z})\right) \cong L^{p}\left(\mu^{(n)}, \mathcal{Z}^{(n)}\right),
$$

it is easy to verify that $\pi_{T}^{(n)}$ is (equivalent to) the integrated form of the representation $T^{(n)}$. We omit the details. 
3.4. Representations of étale groupoids on $L^{p}$-bundles. In this section, we want to isolate a particularly important and natural class of representations of an étale groupoid on Banach spaces.

We fix a quasi-invariant measure $\mu$ on $G^{0}$. Let $\lambda$ be a $\sigma$-finite Borel measure on a standard Borel space $Z$ fibred over $G^{0}$ via $q$, and assume that $\mu=q_{*}(\lambda)$. Denote by $\mathcal{Z}$ the $L^{p}$-bundle $\bigsqcup_{x \in G^{0}} L^{p}\left(\lambda_{x}\right)$ over $\mu$ obtained from the disintegration $\lambda=\int \lambda_{x} d \mu(x)$ as in Theorem 2.11.

Definition 3.23. Adopt the notation from the comments above. A representation $T: G \rightarrow \operatorname{Iso}(\mathcal{Z})$ is called an $L^{p}$-representation of $G$ on $\mathcal{Z}$. Under the identification $L^{p}(\mu, \mathcal{Z}) \cong L^{p}(\mu)$ given by Theorem 2.11, the integrated form $\pi_{T}: C_{c}(G) \rightarrow B\left(L^{p}(\lambda)\right)$ of $T$, is an $I$-norm contractive nondegenerate representation.

It will be shown in Theorem 6.9 that every $I$-norm contractive nondegenerate representation of $C_{c}(G)$ on an $L^{p}$-space is the integrated form of some $L^{p}$-representation of $G$.

Remark 3.24. It is clear that an $L^{2}$-representation of $G$ in the sense of Definition 3.23, is a representation of $G$ on a Borel Hilbert bundle. Conversely, any representation of $G$ on a Borel Hilbert bundle is equivalent - as in Definition 3.13 - to an $L^{2}$-representation. In fact, if $\mathcal{H}$ is a Borel Hilbert bundle over $G^{0}$, then for every $0 \leq \alpha \leq \omega$ the set $X_{\alpha}=\left\{x \in G^{0}: \operatorname{dim}\left(\mathcal{H}_{x}\right)=\alpha\right\}$ is Borel. Thus, $\mathcal{H}$ is isomorphic to the Hilbert bundle

$$
\mathcal{Z}_{0}=\bigsqcup_{0 \leq \alpha \leq \omega} X_{\alpha} \times \ell^{2}(\alpha)
$$

Set $Z=\bigsqcup_{0 \leq \alpha \leq \omega}\left(Z_{\alpha} \times \alpha\right)$, and define a $\sigma$-finite Borel measure $\lambda$ on $Z$ by $\lambda=$ $\bigsqcup_{0 \leq \alpha \leq \omega}\left(\mu \times c_{\alpha}\right)$. It is immediate that $\mathcal{Z}_{0}$ is (isomorphic to) the Borel Hilbert bundle $\bigsqcup_{x \in G^{0}} L^{2}\left(\lambda_{x}\right)$ obtained from the disintegration of $\lambda$ with respect to $\mu$.

In view of the above remark, there is no difference, up to equivalence, between $L^{2}$-representations and representations on Borel Hilbert bundles. The theory of $L^{p}$-representations of $G$ for $p \in(1, \infty)$ can therefore be thought of as a generalization of the theory of representations of $G$ on Borel Hilbert bundles.

Example 3.25 (Left regular representation). Take $Z=G$ and $\lambda=\nu$, in which case the disintegration of $\lambda$ with respect to $\mu$ is $\left(c_{x} G\right)_{x \in X}$. For $\gamma \in G$, define the surjective linear isometry

$$
T_{\gamma}^{\mu, p}: \ell^{p}(s(\gamma) G) \rightarrow \ell^{p}(r(\gamma) G)
$$

by

$$
\left(T_{\gamma}^{\mu, p} \xi\right)(\rho)=\xi\left(\gamma^{-1} \rho\right)
$$


The assignment $\gamma \mapsto T_{\gamma}^{\mu, p}$ defines a representation

$$
T^{\mu, p}: G \rightarrow \text { Iso }\left(\bigsqcup_{x \in G^{0}} \ell^{p}(x G)\right)
$$

which we shall call the left regular $L^{p}$-representation of $G$ associated with $\mu$. When the Hölder exponent $p$ is clear from the context, we will write $T^{\mu}$ in place of $T^{\mu, p}$.

Lemma 3.26. The dual $\left(T^{\mu, p}\right)^{\prime}$ of the left regular $L^{p}$-representation associated with $\mu$ is the left regular $L^{p^{\prime}}$-representation $T^{\mu, p^{\prime}}$ associated with $\mu$.

Proof. The result follows from the following computation, valid for all $\eta \in$ $\ell^{p^{\prime}}(r(\gamma) G)$ and all $\xi \in \ell^{p}(s(\gamma) G)$ :

$$
\begin{aligned}
\left\langle T_{\gamma}^{\mu, p^{\prime}} \eta, \xi\right\rangle & =\sum_{\rho \in r(\gamma) G}\left(T_{\gamma}^{\mu, p^{\prime}} \eta\right)(\rho) \overline{\xi(\rho)}=\sum_{\rho \in r(\gamma) G} \eta\left(\gamma^{-1} \rho\right) \overline{\xi(\rho)} \\
& =\sum_{\theta \in s(\gamma) G} \eta(\theta) \overline{\xi(\gamma \theta)}=\overline{\sum_{\rho \in r\left(\gamma^{-1}\right) G} \xi(\gamma \rho) \overline{\eta(\rho)}} \\
& =\overline{\left\langle T_{\gamma^{-1}}^{\mu, p} \xi, \eta\right\rangle}=\left\langle\left(T_{\gamma^{-1}}^{\mu, p}\right)^{\prime} \eta, \xi\right\rangle .
\end{aligned}
$$

We will now compute the integrated form of the left regular $L^{p}$-representation $T^{\mu}$ of $G$ associated with a quasi-invariant Borel probability measure $\mu$ on $G^{0}$. Following Rieffel's induction theory and for consistency with [26, Section 3.1 and Appendix D] we will denote such representation by $\operatorname{Ind}(\mu)$.

Proposition 3.27. The integrated form $\operatorname{Ind}(\mu)$ of the left regular representation $T^{\mu}$ associated with $\mu$, is the left action of $C_{c}(G)$ on $L^{p}\left(\nu^{-1}\right)$ by convolution.

Proof. It is easy to check that multiplication by $D^{\frac{1}{p}}$ and $D^{\frac{1}{p^{\prime}}}$ define isometric isomorphisms $L^{p}(\nu) \cong L^{p}\left(\nu^{-1}\right)$ and $L^{p^{\prime}}(\nu) \cong L^{p^{\prime}}\left(\nu^{-1}\right)$, respectively.

Given $\xi \in L^{p}(\nu)$ and $\eta \in L^{p^{\prime}}(\nu)$, set $\widehat{\xi}=D^{\frac{1}{p}} \xi \in L^{p}\left(\nu^{-1}\right)$ and $\widehat{\eta}=D^{\frac{1}{p^{\prime}}} \eta \in$ 
$L^{p^{\prime}}\left(\nu^{-1}\right)$. Then

$$
\begin{aligned}
& \langle\operatorname{Ind}(\mu)(f) \xi, \eta\rangle_{L^{p}(\nu)} \\
& =\int f(\gamma)\left\langle T_{\gamma} \xi_{s(\gamma)}, \eta_{r(\gamma)}\right\rangle d \nu(\gamma) \\
& =\int \sum_{\gamma \in x G} f(\gamma)\left\langle T_{\gamma} \xi_{s(\gamma)}, \eta_{x}\right\rangle D^{-\frac{1}{p}}(\gamma) d \mu(x) \\
& =\int \sum_{\gamma \in x G} f(\gamma)\left(\sum_{\rho \in x G} \xi\left(\gamma^{-1} \rho\right) \overline{\eta(\rho)}\right) D^{-\frac{1}{p}}(\gamma) d \mu(x) \\
& =\int \sum_{x \in x G} \sum_{\rho \in x G}\left(\sum_{\gamma \in x G} f(\gamma) \widehat{\xi}\left(\gamma^{-1} \rho\right)\right) \overline{\widehat{\eta}(\rho)} D^{-1}(\rho) d \mu(x) \\
& =\int \sum_{\gamma \in x G} \sum_{\rho \in x G}(f * \widehat{\xi})(\rho) \overline{\widehat{\eta}(\rho)} D^{-1}(\rho) d \mu(x) \\
& =\langle f * \widehat{\xi}, \widehat{\eta}\rangle_{L^{p}\left(\nu^{-1}\right)}
\end{aligned}
$$

This finishes the proof.

Lemma 3.28. A function $f$ in $C_{c}(G)$ belongs to $\operatorname{Ker}(\operatorname{Ind}(\mu))$ if and only if it vanishes on the support of $\nu$.

Proof. Suppose that $f$ vanishes on the support of $\nu$. Then

$$
\langle\operatorname{Ind}(\mu)(f) \xi, \eta\rangle_{L^{p}(\nu)}=\int f(\gamma)\left\langle T_{\gamma} \xi_{s(\gamma)}, \eta_{r(\gamma)}\right\rangle D^{-\frac{1}{p}}(\gamma) d \nu(\gamma)=0 .
$$

for every $\xi \in L^{p}(\nu)$ and $\eta \in L^{p^{\prime}}(\nu)$, so $\operatorname{Ind}(\mu)(f)=0$.

Conversely, if $\operatorname{Ind}(\mu)(f)=0$ then $f * \xi=0$ for every $\xi \in L^{p}\left(\nu^{-1}\right)$. In particular, $f=f * \chi_{G^{0}}=0$ in $L^{p}\left(\nu^{-1}\right)$. Thus $f(\gamma)=0$ for $\nu^{-1}$-almost every $\gamma \in G$ and hence also for $\nu$-almost every $\gamma \in G$. By continuity of $f$, this implies that $f$ vanishes on the support of $\nu$.

Definition 3.29. Let us say that a family $\mathcal{M}$ of quasi-invariant probability measures on $G^{0}$ separates points, if for every nonzero function $f \in C_{c}(G)$, there is a measure $\mu \in \mathcal{M}$ such that $f$ does not vanish on the support of the integrated measure $\nu=\int c_{x} G d \mu(x)$. Similarly, a collection $\mathcal{R}$ of representations of $C_{c}(G)$ on Banach algebras is said to separate points if for every nonzero function $f \in C_{c}(G)$, there is a representation $\pi \in \mathcal{R}$ such that $\pi(f)$ is nonzero.

By Lemma 3.28, a family $\mathcal{M}$ of Borel probability measures on $G^{0}$ separates points if and only if the collection of left regular representations associated with elements of $\mathcal{M}$ separates points.

Proposition 3.30. The family of left regular representations associated with quasi-invariant Borel probability measures on $G^{0}$ separates points. 
Proof. A quasi-invariant Borel probability measure is said to be transitive if it is supported by an orbit. Every orbit carries a transitive measure, which is unique up to equivalence; see [37, Definition 3.9 of Chapter 1 ]. It is well known that the transitive measures constitute a collection of quasi-invariant Borel probability measures on $G^{0}$ that separates points; see [37, Proposition 1.11 of Chapter 2], so the proof is complete.

\section{Representations of InVERSE SEMigroups ON $L^{p}$-SPACES}

4.1. The Banach-Lamperti theorem. Let $\mu$ and $\nu$ be Borel probability measures on standard Borel spaces $X$ and $Y$, and let $p \in[1, \infty)$. For $f \in$ $L^{p}(\mu)$, the support of $f$, denoted $\operatorname{supp}(f)$, is the largest element $F$ of $\mathcal{B}_{\mu}$ such that $f \chi_{F}=f$. (See Subsection 1.1 for the definition of the Boolean algebra $\mathcal{B}_{\mu}$.) The completeness of $\mathcal{B}_{\mu}$ implies that such a largest element exists.

Lemma 4.1 (Lamperti-Clarkson; see [11, Proposition 3.2.2]). Adopt the notation of the comments above, and suppose that $p \neq 2$. If $f, g \in L^{p}(\mu)$ satisfy

$$
\|f+g\|^{p}+\|f-g\|^{p}=2\|f\|^{p}+2\|g\|^{p},
$$

then the supports of $f$ and $g$ are disjoint elements of $\mathcal{B}_{\mu}$.

Theorem 4.2 (Banach-Lamperti). Let $p \in[1, \infty) \backslash\{2\}$. If $u: L^{p}(\mu) \rightarrow$ $L^{p}(\nu)$ is a surjective linear isometry, then there are conull Borel subsets $X_{0}$ and $Y_{0}$ of $X$ and $Y$, a Borel isomorphism $\phi: X_{0} \rightarrow Y_{0}$ such that $\left.\phi_{*}(\mu)\right|_{X_{0}} \sim$ $\left.\nu\right|_{Y_{0}}$, and a Borel function $g: Y \rightarrow \mathbb{C}$ with $|g(y)|^{p}=\frac{d \phi_{*}(\mu)}{d \nu}(y)$ for $\nu$-almost every $y \in Y$, such that

$$
u \xi=g \cdot\left(\xi \circ \phi^{-1}\right)
$$

for every $\xi \in L^{p}(\nu)$.

Proof. Define maps $\Psi: \mathcal{B}_{\mu} \rightarrow \mathcal{B}_{\nu}$ and $\Phi: \mathcal{B}_{\nu} \rightarrow \mathcal{B}_{\mu}$ by

$$
\Psi(F)=\operatorname{supp}\left(u^{-1} \chi_{F}\right) \text { and } \Phi(E)=\operatorname{supp}\left(u \chi_{E}\right)
$$

for all $F \in \mathcal{B}_{\mu}$ and all $E \in \mathcal{B}_{\nu}$. It follows from Lemma 4.1 that $\Phi$ and $\Psi$ are mutually inverse $\sigma$-complete Boolean algebra homomorphisms. By [17, Theorem 15.10] there are conull subsets $X_{0}$ and $Y_{0}$ of $X$ and $Y$ respectively, and a Borel isomorphism $\phi: X_{0} \rightarrow Y_{0}$ such that $\Phi(E)=\phi^{-1}\left(E \cap Y_{0}\right)$ for every $E \in \mathcal{B}_{\nu}$. It follows that $\left.\phi_{*}\left(\left.\mu\right|_{X_{0}}\right) \sim \nu\right|_{Y_{0}}$. Set $g=u 1_{X}$, and observe that

$$
u \chi_{F}=g \cdot \chi_{\phi(F)}=g \cdot\left(\chi_{F} \circ \phi^{-1}\right)
$$

for every $F \in \mathcal{B}_{\mu}$. Thus $u \xi=g \cdot\left(\xi \circ \phi^{-1}\right)$ for every $\xi \in L^{p}(\mu)$. We conclude that $|g(y)|^{p}=\frac{d \phi_{*}(\mu)}{d \nu}(y)$ for $\nu$-almost every $y \in Y$, and this finishes the proof. 
4.2. Hermitian idempotents and spatial partial isometries. Let $X$ be a complex vector space. The following definition is taken from [22].

Definition 4.3. A semi-inner product on $X$ is a function $[\cdot, \cdot]: X \times X \rightarrow \mathbb{C}$ satisfying:

(1) $[\cdot, \cdot]$ is linear in the first variable;

(2) $[x, \lambda y]=\bar{\lambda}[x, y]$ for every $\lambda \in \mathbb{C}$ and $x, y \in X$;

(3) $[x, x] \geq 0$ for every $x \in X$, and equality holds if and only if $x=0$;

(4) $|[x, y]| \leq[x, x][y, y]$ for every $x, y \in X$.

The norm on $X$ associated with the semi-inner product $[\cdot, \cdot]$ is defined by $\|x\|=[\cdot, \cdot]^{\frac{1}{2}}$ for $x \in X$.

In general, there might be different semi-inner products on $X$ that induce the same norm. Nonetheless, it is not difficult to see that on a smooth Banach space - and in particular on $L^{p}$-spaces - there is at most one semiinner product compatible with its norm; see the remark after the proof of Theorem 3 in 22$]$.

Definition 4.4. A semi-inner product on a Banach space that induces its norm is called compatible. A Banach space $X$ endowed with a compatible semi-inner product is called a semi-inner product space.

By the above discussion, if $X$ is a smooth Banach space, then a compatible semi-inner product — when it exists - is unique.

Remark 4.5. It is easy to verify that the norm of $L^{p}(\lambda)$ is induced by the semi-inner product

$$
[f, g]=\|g\|_{p}^{2-p} \int f(x) \overline{g(x)}|g(x)|^{p-2} d \lambda(x)
$$

for $f, g \in L^{p}(\lambda)$ with $g \neq 0$.

An inner product on $X$ is precisely a semi-inner product such that moreover $[x, y]=\overline{[y, x]}$ for every $x, y \in X$. Semi-inner products allow one to generalize notions for operators on Hilbert spaces to more general Banach spaces.

Definition 4.6. Let $X$ be a semi-inner product space, and let $T \in B(X)$. The numerical range $W(T)$ of $T$, is the set

$$
\{[T x, x]: x \in X,[x, x]=1\} \subseteq \mathbb{C} .
$$

The operator $T$ is called hermitian if $W(T) \subseteq \mathbb{R}$.

Adopt the notation and terminology from the definition above. In view of [22] the following statements are equivalent:

(1) $T$ is hermitian;

(2) $\|1+i r T\| \leq 1+o(r)$ for $r \rightarrow 0$;

(3) $\|\exp (i r T)\|=1$ for all $r \in \mathbb{R}$. 
It is clear that when $X$ is a Hilbert space, an operator is hermitian if and only if it is self-adjoint. In particular, the hermitian idempotents on a Hilbert space are exactly the orthogonal projections.

Let $\lambda$ be a Borel measure on a standard Borel space $Z$. Hermitian idempotents on $L^{p}(\lambda)$, for $p \neq 2$, have been characterized by Banach in [2]: these are precisely the multiplication operators associated with characteristic functions on Borel subsets of $Z$.

Recall that a bounded linear operator $s$ on a Hilbert space is a partial isometry if there is another bounded linear operator $t$ such that st and $t s$ are orthogonal projections. The following is a generalization of partial isometries on Hilbert spaces to $L^{p}$-spaces. We use the term 'spatial' in accordance to the terminology in [27, 30, 29, 28].

Definition 4.7. Let $X$ be a Banach space and $s \in B(X)$. We say that $s$ is a partial isometry if $\|s\| \leq 1$ and there exists $t \in B(X)$ such that $\|t\| \leq 1$ and $s t$ and $t s$ are idempotent.

Definition 4.8. Let $X$ be a semi-inner product space and $s \in B(X)$. We say that $s$ is a spatial partial isometry if $\|s\| \leq 1$ and there exists $t \in B(X)$ such that $\|t\| \leq 1$ and $s t$ and $t s$ are hermitian idempotents.

Following [27, we call an element $t$ as in Definition 4.8 a reverse of $s$. (It is in general not unique.) We call $t s$ and st the source and range idempotents of $s$, respectively. We denote by $\mathcal{S}(B(X))$ the set of all spatial partial isometries in $R$, and by $\mathcal{E}(B(X))$ the set of hermitian idempotents in $R$.

It is a standard fact in Hilbert space theory that all partial isometries on a Hilbert space are spatial. Moreover, the reverse of a partial isometry on a Hilbert space is unique, and it is given by its adjoint. The situation for partial isometries on $L^{p}$-spaces, for $p \neq 2$, is rather different. The following proposition can be taken as a justification for the term "spatial".

Proposition 4.9. Let $p \in(1, \infty) \backslash\{2\}$ and let $\lambda$ be a $\sigma$-finite Borel measure on a standard Borel space $Z$. If $s$ is a spatial partial isometry on $L^{p}(\lambda)$, then there are Borel subsets $E$ and $F$ of $Z$, a Borel isomorphism $\phi: E \rightarrow F$, and a Borel function $g: F \rightarrow \mathbb{C}$ such that

$$
(s \xi)(y)= \begin{cases}g(y) \cdot\left(\xi \circ \phi^{-1}\right)(y) & \text { if } y \in F, \text { and } \\ 0 & \text { otherwise }\end{cases}
$$

for all $\xi$ in $L^{p}(\lambda)$ and for $\lambda$-almost every $y \in Z$.

Moreover, if $e$ is a hermitian idempotent in $L^{p}(\lambda)$, then there is a Borel subset $E$ of $Z$ such that $e=\Delta_{\chi_{E}}$.

Proof. The result follows from the characterization of hermitian idempotents mentioned above, together with Theorem 4.2 . 
Remark 4.10. Adopt the notation of the above proposition. It is easy to check that the reverse of $s$ is also spatial, and that it is given by

$$
(t \xi)(y)=\left\{\begin{array}{lc}
\overline{(g \circ \phi)(y)} \cdot(\xi \circ \phi)(y) & \text { if } y \in E, \text { and } \\
0 & \text { otherwise }
\end{array}\right.
$$

for all $\xi$ in $L^{p}(\lambda)$ and for $\lambda$-almost every $y \in Z$. In particular, the reverse of a spatial partial isometry of an $L^{p}$-space is unique. We will consequently write $s^{*}$ for the reverse of a spatial partial isometry $s$.

The set $\mathcal{S}\left(L^{p}(\lambda)\right)$ of spatial partial isometries on $L^{p}(\lambda)$ is an inverse semigroup, and the set $\mathcal{E}\left(L^{p}(\lambda)\right)$ of hermitian idempotents on $L^{p}(\lambda)$ is precisely the semilattice of idempotent elements of $\mathcal{S}\left(L^{p}(\lambda)\right)$. Moreover, the map $\mathcal{B}_{\lambda} \rightarrow \mathcal{E}\left(L^{p}(\lambda)\right)$ given by $F \mapsto \Delta_{\chi_{F}}$ is an isomorphism of semilattices. Thus, $\mathcal{E}\left(L^{p}(\lambda)\right)$ is a complete Boolean algebra.

Remark 4.11. If $\left(e_{j}\right)_{j \in I}$ is an increasing net of hermitian idempotents, then $\sup e_{j}$ is the limit of the sequence $\left(e_{j}\right)_{j \in I}$ in the strong operator topology. $j \in I$

4.3. Representations of inverse semigroups. We now turn to inverse semigroup representations on $L^{p}$-spaces by spatial partial isometries. Fix an inverse semigroup $\Sigma$, and recall that $M_{n}(\Sigma)$ has a natural structure of inverse semigroup for every $n \geq 1$ by [26, Proposition 2.1.4].

Definition 4.12. Let $\lambda$ be a $\sigma$-finite Borel measure on a standard Borel space. A representation of $\Sigma$ on $L^{p}(\lambda)$ is a semigroup homomorphism $\rho: \Sigma \rightarrow \mathcal{S}\left(L^{p}(\lambda)\right)$.

For $n \geq 1$ denote by $\lambda^{(n)}$ the measure $\lambda \times c_{n}$, where $c_{n}$ is the counting measure on $n$. We define the amplification $\rho^{(n)}: M_{n}(\Sigma) \rightarrow \mathcal{S}\left(L^{p}\left(\lambda^{(n)}\right)\right)$ of $\rho$, by $\rho^{n}\left(\left[\sigma_{i j}\right]_{i, j \in n}\right)=\left[\rho\left(\sigma_{i j}\right)\right]_{i, j \in n}$, where we identify $B\left(L^{p}\left(\lambda^{(n)}\right)\right)$ with $M_{n}\left(B\left(L^{p}(\lambda)\right)\right)$ in the usual way.

The dual of $\rho$ is the representation $\rho^{\prime}: \Sigma \rightarrow \mathcal{S}\left(L^{p^{\prime}}(\lambda)\right)$ given by $\rho^{\prime}(\sigma)=$ $\rho\left(\sigma^{*}\right)^{\prime}$ for $\sigma \in \Sigma$.

Definition 4.13. Denote by $\mathbb{C} \Sigma$ complex *-algebra of formal linear combinations of elements of $\Sigma$, with operations determined by $\delta_{\sigma} \delta_{\tau}=\delta_{\sigma \tau}$ and $\delta_{\sigma}^{*}=\delta_{\sigma^{*}}$ for all $\sigma, \tau \in \Sigma$, and endowed with the $\ell^{1}$-norm. The canonical identification of $\mathbb{C} M_{n}(\Sigma)$ with $M_{n}(\mathbb{C} \Sigma)$ for $n \geq 1$, defines matrix norms on $\mathbb{C} \Sigma$.

Remark 4.14. Every representation $\rho: \Sigma \rightarrow \mathcal{S}\left(L^{p}(\lambda)\right)$ induces a contractive representation $\pi_{\rho}: \mathbb{C} \Sigma \rightarrow B\left(L^{p}(\lambda)\right)$ such that $\pi_{\rho}\left(\delta_{\sigma}\right)=\rho(\sigma)$ for $\sigma \in \Sigma$. It is not difficult to verify the following facts:

(1) Since, for $n \geq 1$, the amplification $\pi_{\rho}^{(n)}$ of $\pi_{\rho}$ to $M_{n}(\mathbb{C} \Sigma)$ is the representation associated with the amplification $\rho^{(n)}$ of $\rho$, it follows that $\pi_{\rho}$ is $p$-completely contractive.

(2) The representation $\pi_{\rho^{\prime}}$ associated with the dual $\rho^{\prime}$ of $\rho$ is the dual of the representation $\pi_{\rho}$ associated with $\rho$. 
Definition 4.15. Let $\lambda$ and $\mu$ be $\sigma$-finite Borel measure on standard Borel spaces, and let $\rho$ and $\kappa$ be representations of $\Sigma$ on $L^{p}(\lambda)$ and $L^{p}(\mu)$ respectively. We say that $\rho$ and $\kappa$ are equivalent if there is a surjective linear isometry $u: L^{p}(\lambda) \rightarrow L^{p}(\mu)$ such that $u \rho(\sigma)=\kappa(\sigma) u$ for every $\sigma \in \Sigma$.

Adopt the notation of the definition above. If $\rho$ and $\kappa$ are equivalent, then their dual representations $\rho^{\prime}$ and $\kappa^{\prime}$ are also equivalent. Similarly, if $\rho$ and $\kappa$ are equivalent, then the corresponding representations $\pi_{\rho}$ and $\pi_{\kappa}$ of $\mathbb{C} \Sigma$ are equivalent.

4.4. Tight representations of semilattices. In the following, all semilattices will be assumed to have a minimum element 0. Consistently, all inverse semigroups will be assumed to have a neutral element 0 , which is the minimum of the associated idempotent semilattice. In the rest of this subsection we recall some definitions from Section 11 of [10].

Definition 4.16. Let $E$ be a semilattice and let $\mathcal{B}=(\mathcal{B}, 0,1, \wedge, \vee, \neg)$ be a Boolean algebra. A representation of $E$ on $\mathcal{B}$ is a semilattice morphism $E \rightarrow(\mathcal{B}, \wedge)$ satisfying $\beta(0)=0$.

Two elements $x, y$ of $E$ are said to be orthogonal, written $x \perp y$, if $x \wedge y=$ 0 . Furthermore, we say that $x$ and $y$ intersect (each other) if they are not orthogonal.

If $X \subseteq Y \subseteq E$, then $X$ is a cover for $Y$ if every nonzero element of $Y$ intersects an element of $X$.

It is easy to verify that a representation of a semilattice $E$ on a Boolean algebra sends orthogonal elements to orthogonal elements. It is also immediate to check that a cover for the set of predecessors of some $x \in E$ is also a cover for $\{x\}$.

Notation 4.17. If $X$ and $Y$ are (possibly empty) subsets of $E$, we denote by $E^{X, Y}$ the set

$$
E^{X, Y}=\{z \in E: z \leq x \text { for all } x \in X, \text { and } z \perp y \text { for all } y \in Y\} .
$$

We are now ready to state the definition of tight representation of a semilattice.

Definition 4.18. Let $E$ be a semilattice and let $\mathcal{B}$ be a Boolean algebra. A representation $\beta: E \rightarrow \mathcal{B}$ is said to be tight if for every pair $X, Y$ of (possibly empty) finite subsets of $E$ and every finite cover $Z$ of $E^{X, Y}$, we have

$$
\bigvee_{z \in Z} \beta(z)=\bigwedge_{x \in X} \beta(x) \wedge \bigwedge_{y \in Y} \neg \beta(y)
$$

Lemma 4.19. Let $E$ be a semilattice, let $\mathcal{B}$ be a Boolean algebra and let $\beta: E \rightarrow \mathcal{B}$ be a tight representation. If $z_{0}, \ldots, z_{n-1}$ are elements of $E$ such that for every $w \in E$, there exists $j \in n$ such that $z_{j} \wedge w \neq 0$, then $\bigvee_{j \in n} \beta\left(z_{j}\right)=1$. In particular, if $E$ has a largest element 1 , then $\beta(1)=1$. 
Proof. The result is immediate since the assumptions imply that $\left\{z_{0}, \ldots, z_{n-1}\right\}$ is a cover of $E^{\varnothing, \varnothing}$.

Lemma 4.20 ([10, Proposition 11.9]). Suppose that $E$ is a Boolean algebra. If $\beta$ is a representation of $E$ on $\mathcal{B}$, then $\beta$ is tight if and only if $\beta$ is a Boolean algebra homomorphism.

Definition 4.21. Suppose that $E$ is a semilattice. A subsemilattice $F$ of $E$ is dense if for every $x \in E$ nonzero there is $y \in F$ nonzero such that $y \leq x$.

Lemma 4.22. Suppose that $E$ is a semilattice, and $F$ is a dense subsemilattice of $E$. If $\beta$ is a tight representation of $E$ on $\mathcal{B}$, then the restriction of $\beta$ to $F$ is a tight representation of $F$.

Proof. Suppose that $X, Y \subset F$ and that $Z$ is a cover for $F^{X, Y}$. We claim that $Z$ is a cover for $E^{X, Y}$. Pick $x \in E^{X, Y}$ nonzero. Then there is $y \in F$ nonzero such that $y \leq x$. Since $y \in F^{X, Y}$ and $Z$ is a cover for $F^{X, Y}$, there is $z \in Z$ such that $z$ and $y$ intersect. Therefore also $z$ and $x$ intersect. This shows that $Z$ is a cover for $E^{X, Y}$. Therefore Equation (44) holds. This concludes the proof that the restriction of $\beta$ to $F$ is tight.

4.5. Tight representations of inverse semigroups on $L^{p}$-spaces. As in the case of representation of inverse semigroups on Hilbert spaces (see [10. Section 13]), we will isolate a class of "well behaved" representations of inverse semigroups on $L^{p}$-spaces. The following definition is a natural generalization of [10, Definition 13].

Definition 4.23. Let $\lambda$ be a $\sigma$-finite Borel measure on a standard Borel space. A representation $\rho: \Sigma \rightarrow \mathcal{S}\left(L^{p}(\lambda)\right)$ is said to be tight if its restriction to the idempotent semilattice $E(\Sigma)$ of $\Sigma$ is a tight representation on the Boolean algebra $\mathcal{E}\left(L^{p}(\lambda)\right)$ of hermitian idempotents.

Remark 4.24. If $\rho: \Sigma \rightarrow \mathcal{S}\left(L^{p}(\lambda)\right)$ is a tight representation as above, then the net $(\rho(\sigma))_{\sigma \in E(\Sigma)}$ converges to the identity in the strong operator topology. Thus, tightness should be thought of as a nondegeneracy condition for representations of inverse semigroups

Definition 4.25. A tight representation $\rho$ of $\Sigma$ on $L^{p}(\lambda)$ is said to be regular if, for every idempotent open slice $U$ of $G, \rho(U)$ is the limit of the net $(\rho(V))_{V}$ where $V$ ranges among all idempotent open slices of $G$ with compact closure contained in $U$, ordered by inclusion. In formulas

$$
\rho(U)=\lim _{V \in E\left(\Sigma_{c}(G)\right), \bar{V} \subseteq U} \rho(V) .
$$

4.6. Representations of semigroups of slices. Let $G$ be an étale groupoid, let $\lambda$ be a $\sigma$-finite Borel measure on a standard Borel space, and let $\pi$ be a contractive nondegenerate representation of $C_{c}(G)$ on $L^{p}(\lambda)$. Denote by $\Sigma_{c}(G)$ the inverse semigroup of precompact open slices of $G$. In this subsection, we show how to associate to $\pi$ a tight, regular representation of $\rho_{\pi}$ of $\Sigma_{c}(G)$ on $L^{p}(\lambda)$. 
Given a precompact open slice $A$ of $G, \xi \in L^{p}(\lambda)$, and $\eta \in L^{p^{\prime}}(\lambda)$, the assignment $f \mapsto\langle\pi(f) \xi, \eta\rangle$ is a $\|\cdot\|_{\infty}$-continuous linear functional on $C_{c}(A)$ of norm at most $\|\xi\|\|\eta\|$. By the Riesz-Markov-Kakutani representation theorem, there is a Borel measure $\mu_{A, \xi, \eta}$ supported on $A$, of total mass at most $\|\xi\|\|\eta\|$, such that

$$
\langle\pi(f) \xi, \eta\rangle=\int f d \mu_{A, \xi, \eta}
$$

for every $f \in C_{c}(G)$. If $A, B \in \Sigma_{c}(G)$, then $\mu_{A, \xi, \eta}$ and $\mu_{B, \xi, \eta}$ coincide on $A \cap B$. Arguing as in [26, page 87, and pages 98-99], we conclude that there is a Borel measure $\mu_{\xi, \eta}$ defined on all of $G$, such that $\mu_{A, \xi, \eta}$ is the restriction of $\mu_{\xi, \eta}$ to $A$, for every $A \in \Sigma_{c}(G)$, and moreover $\langle\pi(f) \xi, \eta\rangle=\int f d \mu_{\xi, \eta}$ for every $f \in C_{c}(G)$.

Lemma 4.26. The linear span of $\left\{\pi\left(\chi_{A}\right) \xi: A \in \Sigma_{c}(G), \xi \in L^{p}(\lambda)\right\}$ is dense in $L^{p}(\lambda)$.

Proof. Let $\eta \in L^{p^{\prime}}(\lambda)$ satisfy $\left\langle\rho_{\pi}(A) \xi, \eta\right\rangle=0$ for every $\xi \in L^{p}(\lambda)$ and every $A \in \Sigma_{c}(G)$. Since $\left\langle\rho_{\pi}(A) \xi, \eta\right\rangle=\int \chi_{A} d \mu_{\xi, \eta}$, we conclude that $\mu_{\xi, \eta}(A)=0$ for every $\xi \in L^{p}(\lambda)$ and every $A \in \Sigma_{c}(G)$. Thus $\langle\pi(f) \xi, \eta\rangle=0$ for every $f \in C_{c}(G)$ and every $\xi \in L^{p}(\lambda)$. Since $\pi$ is nondegenerate, we conclude that $\eta=0$, which finishes the proof.

Equation (6) allows one to extend $\pi$ to $B_{c}(G)$ by defining

$$
\langle\pi(f) \xi, \eta\rangle=\int f d \mu_{\xi, \eta}
$$

for $f \in B_{c}(G), \xi \in L^{p}(\lambda)$, and $\eta \in L^{p}(\eta)$. Lemma 2.2.1 of [26] shows that $\pi$ is indeed a nondegenerate representation of $B_{c}(G)$ on $L^{p}(\lambda)$. In particular the function $\rho_{\pi}: A \mapsto \pi\left(\chi_{A}\right)$ is a semigroup homomorphism from $\Sigma_{c}(G)$ to $B\left(L^{p}(\lambda)\right)$. We will show below that such a function is a tight, regular representation of $\Sigma_{c}(G)$ on $L^{p}(\lambda)$

Suppose that $f \in B\left(G^{0}\right)$. Define $\pi(f) \in B\left(L^{p}(\lambda)\right)$ by

$$
\langle\pi(f) \xi, \eta\rangle=\int f d \mu_{\xi, \eta}
$$

for $\xi \in L^{p}(\lambda)$, and $\eta \in L^{p^{\prime}}(\lambda)$. Since

$$
\pi(f g)=\pi(f) \pi(g)
$$

for $f, g \in B_{c}\left(G^{0}\right)$, it follows via a monotone classes argument that Equation (8) holds for any $f, g \in B\left(G^{0}\right)$. In particular, $\pi\left(\chi_{A}\right)$ is an idempotent for every $A \in \mathcal{B}(G)$. It follows from Lemma 4.26 that $\pi\left(\chi_{G^{0}}\right)$ is the identity operator on $L^{p}(\lambda)$. Fix now $A \in \mathcal{B}\left(G^{0}\right)$ and $r \in \mathbb{R}$. For any $\xi \in L^{p}(\lambda)$ and 
$\eta \in L^{p^{\prime}}(\lambda)$ we have that

$$
\begin{aligned}
\left|\left\langle\left(1+i r \pi\left(\chi_{A}\right)\right) \xi, \eta\right\rangle\right| & =\left|\left\langle\pi\left(\chi_{G^{0}}+i r \chi_{A}\right) \xi, \eta\right\rangle\right| \\
& =\left|\int\left(\chi_{G^{0}}+i r \chi_{A}\right) d \mu_{\xi, \eta}\right| \\
& \leq\left\|\chi_{G^{0}}+i r \chi_{A}\right\|_{\infty}\|\xi\|\|\eta\| \\
& \leq\left(1+\frac{1}{2} r^{2}\right)\|\xi\|\|\eta\| .
\end{aligned}
$$

Therefore

$$
\left\|1+i r \pi\left(\chi_{A}\right)\right\| \leq 1+\frac{1}{2} r^{2} .
$$

This shows that $\pi\left(\chi_{A}\right)$ is an hermitian idempotent of $L^{p}(\lambda)$.

It follows from Equation (77) and Equation (8) that the function $A \mapsto$ $\pi\left(\chi_{A}\right)$ is a $\sigma$-complete homomorphism of Boolean algebras from $\mathcal{B}\left(G^{0}\right)$ to $\mathcal{E}\left(L^{p}(\lambda)\right)$. Therefore by Lemma 4.20 and Lemma 4.22 the function $\rho_{\pi}: A \mapsto$ $\pi\left(\chi_{A}\right)$ for $A \in \Sigma_{c}(G)$ is a tight, regular representation of $\Sigma_{c}(G)$ on $L^{p}(\lambda)$.

The same argument shows that if $\Sigma$ is an inverse subsemigroup of $\Sigma_{c}(G)$ which is a basis for the topology of $G$, then the restriction of $\rho_{\pi}$ to $\Sigma$ is a tight, regular representation of $\Sigma$ on $L^{p}(\lambda)$.

Remark 4.27. It is clear that if $\pi$ and $\widetilde{\pi}$ are $I$-norm contractive nondegenerate representations of $C_{c}(G)$ on $L^{p}$-spaces, then $\pi$ and $\tilde{\pi}$ are equivalent if and only if $\rho_{\pi}$ and $\rho_{\tilde{\pi}}$ are equivalent. The easy details are left to the reader.

\section{Disintegration of Representations}

Throughout this section, we let $G$ be a locally compact groupoid and $\Sigma$ be an inverse subsemigroup of $\Sigma_{c}(G)$ that generates the topology of $G$. Denote by $\Sigma_{c}$ the inverse semigroup of precompact elements of $\Sigma$. Let $\lambda$ be a $\sigma$-finite measure on a standard Borel space $Z$.

\subsection{The disintegration theorem.}

Theorem 5.1. If $\rho: G \rightarrow \mathcal{S}\left(L^{p}(\lambda)\right)$ is a tight, regular representation, then there are a quasi-invariant measure $\mu$ on $G^{0}$, and, with $\lambda=\int \lambda_{x} d \mu(x)$ denoting the disintegration of $\lambda$ with respect to $\mu$, a representation $T$ of $G$ on the Borel Banach bundle $\bigsqcup_{x \in G^{0}} L^{p}\left(\lambda_{x}\right)$, such that

$$
\langle\rho(A) \xi, \eta\rangle=\int_{A} D(\gamma)^{-\frac{1}{p}}\left\langle T_{\gamma} \xi_{s(\gamma)}, \eta_{r(\gamma)}\right\rangle d \nu(\gamma)
$$

for $A \in \Sigma$, for $\xi \in L^{p}(\lambda)$, and for $\eta \in L^{p^{\prime}}(\lambda)$.

The rest of this section is dedicated to the proof of the theorem above. For simplicity and without loss of generality, we will focus on the case where $\lambda$ is a probability measure. In the following, we fix a representation $\rho$ as in the statement of Theorem 5.1 . 
5.2. Fibration. Define $\Phi: E(\Sigma) \rightarrow \mathcal{B}_{\lambda}$ by $\Delta_{\chi_{\Phi(U)}}=\rho(U)$ for $U \in E(\Sigma)$. Denote by $\mathcal{U}$ the semilattice of open subsets of $G^{0}$. Extend $\Phi$ to a function $\mathcal{U} \rightarrow \mathcal{B}_{\lambda}$ by setting

$$
\Phi(V)=\bigcup_{W \in E\left(\Sigma_{c}\right), \bar{W} \subseteq U} \Phi(W) .
$$

Then $\Delta_{\chi_{\Phi(V)}}$ is the limit in the strong operator topology of the increasing net $\left(\Delta_{\chi_{\rho(W)}}\right)_{W \in E\left(\Sigma_{c}\right), \bar{W} \subseteq V}$. By Equation (5), the expression above indeed defines an extension of $\bar{\Phi}$. Moreover, a monotone classes argument shows that $\Phi$ is a representation. Tightness of $\rho$ together with Equation (5) further imply that $\Phi(U \cup V)=\Phi(U) \cup \Phi(V)$ whenever $U$ and $V$ are disjoint, and that

$$
\Phi\left(\bigcup_{n \in \omega} U_{n}\right) \subseteq \bigcup_{n \in \omega} \Phi\left(U_{n}\right)
$$

for any sequence $\left(U_{n}\right)_{n \in \omega}$ in $\mathcal{U}$. For $U \in \mathcal{U}$, set $m(U)=\lambda(\Phi(U))$. Using [26, Proposition 3.2.7], one can extend $m$ to a Borel measure on $G^{0}$ by setting

$$
m(E)=\inf \{m(U): U \in \mathcal{U}, E \subseteq U\}
$$

for $E \in \mathcal{B}\left(G^{0}\right)$. Extend $\Phi$ to a homomorphism from $\mathcal{B}\left(G^{0}\right)$ to $\mathcal{B}_{\lambda}$, by setting

$$
\Phi(E)=\bigwedge\{\Phi(U): U \in \mathcal{U}, U \supseteq E\} .
$$

(The infimum exists by completeness of $\mathcal{B}_{\lambda}$.)

Lemma 5.2. The map $\Phi: \mathcal{B}\left(G^{0}\right) \rightarrow \mathcal{B}_{\lambda}$ is a $\sigma$-complete Boolean algebra homomorphism.

Proof. We claim that given $E_{0}$ and $E_{1}$ in $\mathcal{B}\left(G^{0}\right)$, we have $\Phi\left(E_{0} \cap E_{1}\right)=$ $\Phi\left(E_{0}\right) \cap \Phi\left(E_{1}\right)$.

To prove the claim, observe that if $U_{j}$ is an open set containing $E_{j}$ for $j \in\{0,1\}$, then $\Phi\left(U_{0} \cap U_{1}\right)=\Phi\left(U_{0}\right) \cap \Phi\left(U_{1}\right)$, and thus $\Phi\left(E_{0} \cap E_{1}\right) \subseteq$ $\Phi\left(E_{0}\right) \cap \Phi\left(E_{1}\right)$. In order to prove that equality holds, it is enough to show that given $\varepsilon>0$, we have

$$
\lambda\left(\Phi\left(E_{0} \cap E_{1}\right)\right) \geq \lambda\left(\Phi\left(E_{0}\right) \cap \Phi\left(E_{1}\right)\right)-\varepsilon .
$$

Fix an open set $U$ containing $E_{0} \cap E_{1}$ such that $m(U) \leq m\left(E_{0} \cap E_{1}\right)+\varepsilon$. Let $V_{0}$ and $V_{1}$ be open sets satisfying $E_{j} \backslash\left(E_{0} \cap E_{1}\right) \subseteq V_{j}$ for $j=0,1$, and

$$
\mu\left(V_{j}\right) \leq \mu\left(E_{j} \backslash\left(E_{0} \cap E_{1}\right)\right)+\varepsilon .
$$

For $j=0,1$, set $U_{j}=U \cup V_{j}$. Then $U_{j} \supseteq E_{j}$ and

$$
\begin{aligned}
\lambda\left(\Phi\left(E_{0} \cap E_{1}\right)\right) & =m\left(E_{0} \cap E_{1}\right) \geq m(U)-\varepsilon \geq m\left(U_{0} \cap U_{1}\right)-3 \varepsilon \\
& =\lambda\left(\Phi\left(U_{0} \cap U_{1}\right)\right)-3 \varepsilon=\lambda\left(\Phi\left(U_{0}\right) \cap \Phi\left(U_{1}\right)\right)-3 \varepsilon \\
& \geq \lambda\left(\Phi\left(E_{0}\right) \cap \Phi\left(E_{1}\right)\right)-3 \varepsilon .
\end{aligned}
$$

We have therefore shown that $\Phi\left(E_{0} \cap E_{1}\right)=\Phi\left(E_{0}\right) \cap \Phi\left(E_{1}\right)$, so the claim is proved. 
It remains to show that if $\left(E_{n}\right)_{n \in \omega}$ is a sequence of pairwise disjoint Borel subsets of $G^{0}$, then

$$
\Phi\left(\bigcup_{n \in \omega} E_{n}\right)=\bigcup_{n \in \omega} \Phi\left(E_{n}\right) .
$$

By Equation (9), the left-hand side is contained in the right-hand side. On the other hand, we have

$$
\begin{aligned}
\lambda\left(\Phi\left(\bigcup_{n \in \omega} E_{n}\right)\right) & =m\left(\bigcup_{n \in \omega} E_{n}\right)=\sum_{n \in \omega} m\left(E_{n}\right) \\
& =\sum_{n \in \omega} \lambda\left(\Phi\left(E_{n}\right)\right)=\lambda\left(\bigcup_{n \in \omega} \Phi\left(E_{n}\right)\right)
\end{aligned}
$$

so we conclude that equality holds, and the proof is complete.

By [17, Theorem 15.9], there is a Borel function $q: Z \rightarrow G^{0}$ such that $\Phi(E)=q^{-1}(E)$ for every $E \in \mathcal{B}\left(G^{0}\right)$. Moreover, the map $q$ is unique up to $\lambda$-almost everywhere equality.

5.3. Measure. Define a Borel probability measure $\mu$ on $G^{0}$ by $\mu=q_{*}(\lambda)$. Consider the disintegration $\lambda=\int \lambda_{x} d \mu(x)$ of $\lambda$ with respect to $\mu$, the Borel Banach bundle $\mathcal{Z}=\bigsqcup_{x \in G^{0}} L^{p}\left(\lambda_{x}\right)$, and identify $L^{p}(\lambda)$ with $L^{p}(\mu, \mathcal{Z})$ as in Theorem 2.11.

For $A \in \Sigma$, denote by $\theta_{A}: A^{-1} A \rightarrow A A^{-1}$ the homomorphism defined by $\theta_{A}(x)=r(A x)$ for $x \in A^{-1} A$. Since $\rho(A)$ is a spatial partial isometry with domain $\Phi(s(A))$ and range $\Phi(r(A))$, there are a Borel function $g_{A}: \Phi(r(A)) \rightarrow \mathbb{C}$ and a Borel isomorphism $\phi_{A}: \Phi(s(A)) \rightarrow \Phi(r(A))$ such that

$$
(\rho(A) \xi)_{z}=g_{A}(z) \xi\left(\phi_{A}^{-1}(z)\right)
$$

for $z \in \Phi(r(A))$. We claim that $\left(q \circ \phi_{A}\right)(z)=\left(\theta_{A} \circ q\right)(z)$ for $\lambda$-almost every $z \in \Phi(r(A))$.

By the uniqueness assertion in [17, Theorem 15.9], it is enough to show that $\left(\theta_{A} \circ q \circ \phi_{A}^{-1}\right)^{-1}(U)=\Phi(U)$ for every $U \in E(\Sigma)$ with $U \subseteq r(A)$. We have

$\left(\theta_{A} \circ q \circ \phi_{A}^{-1}\right)^{-1}(U)=\left(\phi_{A} \circ q^{-1} \circ \theta_{A}^{-1}\right)(U)=\phi_{A}\left(\Phi\left(\theta_{A}^{-1}(U)\right)\right)=\phi_{A}\left(\Phi\left(A^{-1} U A\right)\right)$.

Given $\xi \in L^{p}\left(\left.\lambda\right|_{\Phi(r(A))}\right)$, set $\eta=\xi \circ \phi_{A}$. Then

$$
\begin{aligned}
\Delta_{\chi_{\phi_{A}\left(\Phi\left(A^{-1} U A\right)\right)}} \xi & =\left(\Delta_{\chi_{\Phi\left(A^{-1} U A\right)}} \eta\right) \circ \phi_{A}^{-1}=\left(\rho\left(A^{-1} U A\right) \eta\right) \circ \phi_{A}^{-1} \\
& =\left(\rho(A)^{-1} \rho(U) \rho(A) \eta\right) \circ \phi_{A}^{-1}=\left(\rho(A)^{-1} \rho(U) g_{A} \xi\right) \circ \phi_{A}^{-1} \\
& =\left(\rho(A)^{-1} \chi_{\Phi(U)} g_{A} \xi\right) \circ \phi_{A}^{-1} \\
& =\left(\left(g_{A} \circ \phi_{A}\right)^{-1}\left(\chi_{\Phi(U)} \circ \phi_{A}\right)\left(g_{A} \circ \phi_{A}\right) \eta\right) \circ \phi_{A}^{-1} \\
& =\chi_{\Phi(U)} \xi=\Delta_{\chi_{\Phi(U)}} \xi .
\end{aligned}
$$


Thus $\Phi(U)=\phi_{A}\left(\Phi\left(A^{-1} U A\right)\right)=\left(\theta_{A} \circ q \circ \phi_{A}^{-1}\right)^{-1}(U)$, and hence $\left(\theta_{A} \circ q \circ\right.$ $\left.\phi_{A}^{-1}\right)(z)=q(z)$ for $\lambda$-almost every $z \in \Phi(r(A))$, as desired. The claim is proved.

It is shown in [26, Proposition 3.2.2] that $\mu$ is quasi-invariant whenever $\left.\left.\left(\theta_{A}\right)_{*} \mu\right|_{s(A)} \sim \mu\right|_{r(A)}$ for every open slice $A$ of $G$. The same proof in fact shows that it is sufficient to check this condition for $A \in \Sigma$. Given $A \in \Sigma$, we have

$$
\begin{aligned}
\left.\mu\right|_{r(A)} & =\left.q_{*} \lambda\right|_{\Phi(r(A))} \sim q_{*}\left(\left.\left(\phi_{A}\right)_{*} \lambda\right|_{\Phi(s(A))}\right)=\left.\left(q \circ \phi_{A}\right)_{*} \lambda\right|_{\Phi(s(A))}=\left.\left(\theta_{A} \circ q\right)_{*} \lambda\right|_{\Phi(s(A))} \\
& =\left(\theta_{A}\right)_{*}\left(\left.q_{*} \lambda\right|_{\Phi(s(A))}\right)=\left.\left(\theta_{A}\right)_{*} \mu\right|_{s(A)},
\end{aligned}
$$

so $\mu$ is quasi-invariant.

5.4. Disintegration. For $x \in G^{0}$, set $Z_{x}=q^{-1}(\{x\})$, and note that $Z_{x}=$ $\Phi(\{x\})$. Given $A \in \Sigma$, regard $\rho(A)$ as a surjective linear isometry

$$
\rho(A): L^{p}\left(\left.\lambda\right|_{\Phi(s(A))}\right) \rightarrow L^{p}\left(\left.\lambda\right|_{\Phi(r(A))}\right) .
$$

Let $\mathcal{Z}$ denote the Borel Banach bundle

$$
\bigsqcup_{x \in G^{0}} L^{p}\left(\lambda_{x}\right)
$$

and identify $L^{p}\left(\left.\lambda\right|_{\Phi(s(A))}\right)$ and $L^{p}\left(\left.\lambda\right|_{\Phi(r(A))}\right)$ with $L^{p}\left(\left.\mu\right|_{s(A)},\left.\mathcal{Z}\right|_{s(A)}\right)$ and $L^{p}\left(\left.\mu\right|_{r(A)},\left.\mathcal{Z}\right|_{r(A)}\right)$, respectively.

If $U \in E(\Sigma)$ satisfies $U \subseteq r(A)$, one uses $\rho\left(A^{-1} U A\right)=\rho(A)^{-1} \rho(U) \rho(A)$ to show that

$$
\Delta_{U} \circ \rho(A)=\rho(A) \circ \Delta_{\theta_{A}(U)} .
$$

By Theorem 2.21, there is a Borel section $x \mapsto T_{x}^{A}$ of $B\left(\left.\mathcal{Z}\right|_{s(A)},\left.\mathcal{Z}\right|_{r(A)}, \theta_{A}\right)$ consisting of invertible isometries, such that

$$
\left.(\rho(A) \xi)\right|_{Z_{y}}=\left.\left(\frac{d\left(\theta_{A}\right)_{*} \mu}{d \mu}(y)\right)^{\frac{1}{p}} T_{\theta_{A}^{-1}(y)}^{A} \xi\right|_{Z_{\phi^{-1}(y)}}
$$

for $\mu$-almost every $y \in r(A)$. Since

$$
\left.(\rho(A) \xi)\right|_{Z_{y}}=\left.\left(g_{A}\right)\right|_{Z_{y}} \cdot\left(\left.\xi\right|_{Z_{y}} \circ\left(\left.\left(\phi_{A}\right)\right|_{Z_{\theta_{A}^{-1}(y)}} ^{Z_{y}}\right)^{-1}\right)
$$

for $\mu$-almost every $y \in r(A)$, we have

$$
T_{x}^{A} \xi=\left.\left(\frac{d\left(\theta_{A}\right)_{*} \mu}{d \mu}\left(\theta_{A}(x)\right)\right)^{\frac{1}{p}}\left(g_{A}\right)\right|_{Z_{\theta_{A}(x)}}\left(\xi \circ\left(\left.\left(\phi_{A}\right)\right|_{Z_{x}} ^{\mid Z_{\theta_{A}(x)}}\right)^{-1}\right)
$$

for $\mu$-almost every $x \in s(A)$. Arguing as in the proof of [26, Theorem 3.2.1], one can see that if $A$ and $B$ are in $\Sigma$ and $U \in E(\Sigma)$, then

- $T_{x}^{A}=T_{x}^{B}$ for $\mu$-almost every $x \in s(A \cap B)$,

- $\left(T_{x}^{A}\right)^{-1}=T_{\theta_{A}(x)}^{A^{-1}}$ for $\mu$-almost every $x \in s(A)$, and

- $T_{x}^{U}$ is the identity operator of $L^{p}\left(\lambda_{x}\right)$ for $\mu$-almost every $x \in U$. 
Moreover, up to discarding a $\nu$-null set, the assignment $T: G \rightarrow \operatorname{Iso}(\mathcal{Z})$ given by $T_{\gamma}=T_{\gamma}^{A}$ for some $A \in \Sigma$ containing $\gamma$, is well defined and determines a representation of $G$ on $\mathcal{Z}$. It is a consequence of Equation (10) that

$$
\langle\rho(A) \xi, \eta\rangle=\int D(x A)^{-\frac{1}{p}}\left\langle T_{x A} \xi_{\theta_{A}^{-1}(x)}, \eta_{x}\right\rangle d \mu(x),
$$

for every $\xi \in L^{p}(\mathcal{Z}, \mu)$ and every $\eta \in L^{p^{\prime}}\left(\mu, \mathcal{Z}^{\prime}\right)$. This concludes the proof of Theorem 5.1 .

\section{6. $L^{p}$-OPERATOR ALGEBRAS OF ÉTALE GROUPOIDS}

Throughout this section, we fix a Hölder exponent $p \in(1, \infty)$.

\section{1. $L^{p}$-operator algebras.}

Definition 6.1. A concrete $L^{p}$-operator algebra is a subalgebra $A$ of $B\left(L^{p}(\lambda)\right)$ for some $\sigma$-finite Borel measure $\lambda$ on a standard Borel space. The identification of $M_{n}(A)$ with a subalgebra of $B\left(L^{p}\left(\lambda^{(n)}\right)\right)$ induces a norm on $M_{n}(A)$. The collection of such norms defines a $p$-operator space structure on $A$ as in [9, Section 4.1]. Moreover the multiplication on $A$ is a $p$-completely contractive bilinear map. (Equivalently $M_{n}(A)$ is a Banach algebra for every $n \in \mathbb{N}$.)

An abstract $L^{p}$-operator algebra is a Banach algebra $A$ endowed with a $p$-operator space structure, which is $p$-completely isometrically isomorphic to a concrete $L^{p}$-operator algebra.

Let $A$ be a separable matricially normed algebra and let $\mathcal{R}$ be a collection of $p$-completely contractive nondegenerate representations of $A$ on $L^{p}$-spaces. Set $I_{\mathcal{R}}=\bigcap_{\pi \in \mathcal{R}} \operatorname{Ker}(\pi)$. Then $I_{\mathcal{R}}$ is an ideal in $A$. Arguing as in [5, Section 1.2.16], the completion $F^{\mathcal{R}}(A)$ of $A / I_{\mathcal{R}}$ with respect to the norm

$$
\|a+I\|=\sup \{\|\pi(a)\|: \pi \in \mathcal{R}\}
$$

for $a \in A$, is a Banach algebra. Moreover, $F^{\mathcal{R}}(A)$ has a natural $p$-operator space structure that makes it into an (abstract) $L^{p}$-operator algebra.

Remark 6.2. If $\mathcal{R}$ separates the points of $A$, then the ideal $I_{\mathcal{R}}$ is trivial, and hence the canonical map $A \rightarrow F^{\mathcal{R}}(A)$ is an injective $p$-completely contractive homomorphism.

Definition 6.3. Let $\mathcal{R}^{p}$ denote be the collection of all $p$-completely contractive nondegenerate representations of $A$ on $L^{p}$-spaces associated with $\sigma$-finite Borel measures on standard Borel spaces. Then $F^{\mathcal{R}^{p}}(A)$ is abbreviated to $F^{p}(A)$, and called the enveloping $L^{p}$-operator algebra of $A$.

Suppose further that $A$ is a matricially normed *-algebra with a completely isometric involution $a \mapsto a^{*}$. If $\pi: A \rightarrow B\left(L^{p}(\lambda)\right)$ is a $p$-completely contractive nondegenerate representation as before, then the dual representation of $\pi$ is the $p^{\prime}$-completely contractive nondegenerate representation $\pi^{\prime}$ 
given by $\pi^{\prime}(a)=\pi\left(a^{*}\right)^{\prime}$ for all $a \in A$.

Let $\mathcal{R}$ be a collection of $p$-completely contractive nondegenerate representations of $A$ on $L^{p}$-spaces, and denote by $\mathcal{R}^{\prime}$ the collection of duals of elements of $\mathcal{R}$. It is immediate that the involution of $A$ extends to a $p$ completely isometric anti-isomorphism $F^{\mathcal{R}}(A) \rightarrow F^{\mathcal{R}^{\prime}}(A)$. Finally, since $\left(\mathcal{R}^{p}\right)^{\prime}=\mathcal{R}^{p^{\prime}}$, the discussion above shows that the involution of $A$ extends to a $p$-completely isometric anti-isomorphism $F^{p}(A) \rightarrow F^{p^{\prime}}(A)$.

6.2. The full $L^{p}$-operator algebra of an étale groupoid. Let $G$ be an étale groupoid.

Definition 6.4. We define the full $L^{p}$-operator algebra $F^{p}(G)$ of $G$ to be the enveloping $L^{p}$-operator algebra of the matricially normed *algebra $C_{c}(G)$.

Remark 6.5. By Proposition 3.30, the family of $p$-completely contractive nondegenerate representations of $C_{c}(G)$ on $L^{p}$-spaces separates the points of $C_{c}(G)$, and hence the canonical map $C_{c}(G) \rightarrow F^{p}(G)$ is injective.

The proof of the following is straightforward, and is left to the reader.

Proposition 6.6. The correspondence sending a $p$-completely contractive representation of $F^{p}(G)$ on an $L^{p}$-space to its restriction to $C_{c}(G)$, is a bijective correspondence between $p$-completely contractive representations of $F^{p}(G)$ on $L^{p}$-spaces and $p$-completely contractive representations of $C_{c}(G)$ on $L^{p}$-spaces.

Definition 6.7. Let $\Sigma$ be an inverse semigroup, and consider the matricially normed *-algebra $\mathbb{C} \Sigma$. Denote by $\mathcal{R}_{\text {tight }}^{p}$ the collection of tight representations of $\Sigma$ on $L^{p}$-spaces. We define the tight enveloping $L^{p}$-operator algebra of $\Sigma$, denoted $F_{\text {tight }}^{p}(\Sigma)$, to be $F^{\mathcal{R}_{\text {tight }}^{p}}(\mathbb{C} \Sigma)$.

Remark 6.8. Since the dual of a tight representation is also tight, it follows that the involution on $\mathbb{C} \Sigma$ extends to a $p$-completely isometric antiisomorphism $F_{\text {tight }}^{p}(\Sigma) \rightarrow F_{\text {tight }}^{p^{\prime}}(\Sigma)$.

Let $\Sigma$ be an inverse semigroup of open slices of $G$ that is a basis for its topology. Let $\mathcal{Z}$ be a Borel Banach bundle over $G^{0}$, and let $(T, \mu)$ be a representation of $G$ on $\mathcal{Z}$. Then $T$ induces a tight representation $\rho_{T}: \Sigma \rightarrow$ $\mathcal{S}\left(L^{p}(\mathcal{Z})\right)$ determined by

$$
\left\langle\rho_{T}(A) \xi, \eta\right\rangle=\int_{r(A)} D^{-\frac{1}{p}}(x A)\left\langle T_{x A} \xi_{\theta_{A}^{-1}(x)}, \eta_{x}\right\rangle d \mu(x)
$$

for all $A \in \Sigma$, for all $\xi \in L^{p}(\mathcal{Z})$ and all $\eta \in L^{p^{\prime}}\left(\mathcal{Z}^{\prime}\right)$. We also denote by $\pi_{T}$ the integrated form of $T$ as in Theorem 3.14 .

It is shown in Subsection 4.6 that a contractive representation $\pi$ of $C_{c}(G)$ on $L^{p}(\lambda)$ induces a tight regular representation $\rho_{\pi}$ of $\Sigma$ on $L^{p}(\lambda)$.

Theorem 6.9. Adopt the notation of the comments above. 
(1) The assignment $T \mapsto \rho_{T}$ determines a bijective correspondence between representations of $G$ on $L^{p}$-bundles and tight regular representations of $\Sigma$ on $L^{p}$-spaces.

(2) The assignment $\pi \mapsto \rho_{\pi}$ determines a bijective correspondence between contractive representations of $C_{c}(G)$ on $L^{p}$-spaces and tight regular representations of $\Sigma$ on $L^{p}$ spaces.

(3) The assignment $T \mapsto \pi_{T}$ assigning is a bijective correspondence between representations of $G$ on $L^{p}$-bundles and contractive representations of $C_{c}(G)$ on $L^{p}$-spaces.

Moreover, the correspondences in (1), (2), and (3) preserve the natural relations of equivalence of representations.

The difference between (1) and (3) above is that in (3) the representations of $\Sigma$ are not necessarily assumed to be regular. In fact this condition is trivially satisfied by any representation in the case of the inverse semigroup of compact open slices.

Proof. (1). This is an immediate consequence of the Disintegration Theorem 5.1 .

(2). Suppose that $\rho$ is a tight representation of $\Sigma$ on $L^{p}(\lambda)$. Applying the Disintegration Theorem 5.1 one obtains a representation $(\mu, T)$ of $G$ on the bundle $\bigsqcup_{x \in G^{0}} L^{p}(\lambda)$ for a disintegration $\lambda=\int \lambda_{x} d \mu(x)$. One can then assign to $\rho$ the integrated form $\pi_{\rho}$ of $(\mu, T)$. It is easy to verify that the maps $\rho \mapsto \pi_{\rho}$ and $\pi \mapsto \rho_{\pi}$ are mutually inverse.

Finally (3) follows from combining (1) and (2).

Observe that when $G$ is ample, and $\Sigma$ is the inverse semigroup of compact open slices, any tight representation of $\Sigma$ on an $L^{p}$-space is automatically regular.

Corollary 6.10. If $A$ is an $L^{p}$-operator algebra, then any contractive homomorphism from $C_{c}(G)$ or $F^{p}(G)$ to $A$ is automatically $p$-completely contractive.

Proof. It is enough to show that any contractive nondegenerate representation of $C_{c}(G)$ on an $L^{p}$-space is $p$-completely contractive. This follows from part (3) of Theorem 6.9, together with the fact that the integrated form of a representation of $G$ on an $L^{p}$-bundle is $p$-completely contractive, as observed in Subsection 3.3.

Corollary 6.11. Adopt the assumptions of Theorem 6.9, and suppose moreover that $G$ is ample. Then the $L^{p}$-operator algebras $F^{p}(G)$ and $F_{\text {tight }}^{p}(\Sigma)$ are $p$-completely isometrically isomorphic. In particular, $F^{p}(G)$ is generated by its spatial partial isometries.

Proof. This follows from part (2) of Theorem 6.9. 
6.3. Reduced $L^{p}$-operator algebras of étale groupoids. Let $\mu$ be a (not necessarily quasi-invariant) Borel probability measure on $G^{0}$, and let $\nu$ be the measure on $G$ associated with $\mu$ as in Subsection 3.1. Denote by $\operatorname{Ind}(\mu): C_{c}(G) \rightarrow B\left(L^{p}\left(\nu^{-1}\right)\right)$ the left action by convolution. Then $\operatorname{Ind}(\mu)$ is contractive and nondegenerate.

Remark 6.12. When $\mu$ is quasi-invariant, the representation $\operatorname{Ind}(\mu)$ is the integrated form of the left regular representation $T^{\mu}$ of $G$ on $\bigsqcup_{x \in G^{0}} \ell^{p}(x G)$ as defined in Subsection 3.27. The same argument as in Lemma 3.28 shows that a function $f$ in $C_{c}(G)$ belongs to $\operatorname{Ker}(\operatorname{Ind}(\mu))$ if and only if $f$ vanishes on the support of $\nu$.

Definition 6.13. Define $\mathcal{R}_{\text {red }}^{p}$ red to be the collection of representations $\operatorname{Ind}(\mu)$ where $\mu$ varies among the Borel probability measures on $G^{0}$. The reduced $L^{p}$-operator algebra $F_{\text {red }}^{p}(G)$ of $G$ is the enveloping $L^{p}$-operator algebra $F^{\mathcal{R}_{\text {red }}^{p}}\left(C_{c}(G)\right)$. The norm on $F_{\text {red }}^{p}(G)$ is denoted by $\|\cdot\|_{\text {red }}$.

By Proposition 3.30, the family $\mathcal{R}_{\text {red }}^{p}$ separates points, and hence the canonical map $C_{c}(G) \rightarrow F_{\text {red }}^{p}(G)$ is injective. It follows that the identity map on $C_{c}(G)$ extends to a canonical $p$-completely contractive homomorphism $F^{p}(G) \rightarrow F_{\text {red }}^{p}(G)$ with dense range.

Remark 6.14. The dual of $\operatorname{Ind}(\mu): C_{c}(G) \rightarrow B\left(L^{p}\left(\nu^{-1}\right)\right)$ is the representation $\operatorname{Ind}(\mu): C_{c}(G) \rightarrow B\left(L^{p^{\prime}}(\nu)\right)$, and thus the involution on $C_{c}(G)$ extends to a $p$-completely isometric anti-isomorphism $F_{\text {red }}^{p}(G) \rightarrow F_{\text {red }}^{p^{\prime}}(G)$.

For $x \in G^{0}$, we write $\delta_{x}$ for its associated point mass measure, and write $\operatorname{Ind}(x)$ in place of $\operatorname{Ind}\left(\delta_{x}\right)$. In this case, $\nu$ is the counting measure $c_{x} G$ on $x G$, and $\nu^{-1}$ is the counting measure $c_{G x}$ on $G x$. Moreover, $\operatorname{Ind}(x)$ is given by

$$
(\operatorname{Ind}(x) f(\xi))(\rho)=\sum_{\gamma \in r(\rho) G} f(\gamma) \xi\left(\gamma^{-1} \rho\right)
$$

for $f \in C_{c}(G), \xi \in L^{p}\left(\nu^{-1}\right)$, and $\rho \in G x$.

Proposition 6.15. Let $\mu$ be a probability measure on $G^{0}$. If $f \in C_{c}(G)$, then

$$
\|\operatorname{Ind}(\mu) f\|=\sup _{x \in \operatorname{supp}(\mu)}\|\operatorname{Ind}(x)(f)\| .
$$

Proof. Denote by $C$ the support of $\mu$ and fix $f \in C_{c}(G)$. Set

$$
M=\sup _{x \in \operatorname{supp}(\mu)}\|\operatorname{Ind}(x)(f)\| .
$$

We will first show that $\|\operatorname{Ind}(\mu) f\| \leq M$. Given $\xi \in L^{p}\left(\nu^{-1}\right)$ and $\eta \in L^{p^{\prime}}\left(\nu^{-1}\right)$ with $\|\xi\|,\|\eta\| \leq 1$, we use Hölder's inequality at the second to last step to get

$$
|\langle\operatorname{Ind}(\mu)(f) \xi, \eta\rangle|=\left|\int(\operatorname{Ind}(\mu)(f) \xi)(\rho) \overline{\eta(\rho)} d \nu^{-1}(\rho)\right|
$$




$$
\begin{aligned}
& =\left|\int_{\gamma \in r(\rho) G} f(\gamma) \xi\left(\gamma^{-1} \rho\right) \overline{\eta(\rho)} d \nu^{-1}(\rho)\right| \\
& =\left|\int_{C} \sum_{\rho \in G x} \sum_{\gamma \in r(\rho) G} f(\gamma) \xi\left(\gamma^{-1} \rho\right) \overline{\eta(\rho)} d \mu(x)\right| \\
& =\left|\int_{C}\left\langle\left.\operatorname{Ind}(x)(f) \xi\right|_{G x},\left.\eta\right|_{G x}\right\rangle d \mu(x)\right| \\
& \leq \int_{C}\left|\left\langle\left.\operatorname{Ind}(x)(f) \xi\right|_{G x},\left.\eta\right|_{G x}\right\rangle\right| d \mu(x) \\
& \leq M \int_{C}\left\|\left.\xi\right|_{G x}\right\|\left\|\left.\eta\right|_{G x}\right\| d \mu(x) \\
& =M \int_{C}\left(\sum_{\gamma \in G x}|\xi(\gamma)|^{p}\right)^{\frac{1}{p}}\left(\sum_{\gamma \in G x}|\eta(\gamma)|^{p^{\prime}}\right)^{\frac{1}{p^{\prime}}} d \mu(x) \\
& \leq M\left(\int_{C} \sum_{\gamma \in G x}|\xi(\gamma)|^{p} d \mu(x)\right)^{\frac{1}{p}}\left(\int_{C} \sum_{g \in G x}|\eta(\gamma)|^{p^{\prime}}\right)^{\frac{1}{p^{\prime}}} \\
& \leq M,
\end{aligned}
$$

which implies that $\|\operatorname{Ind}(\mu) f\| \leq M$, as desired.

Conversely, fix $x \in C$ and let $\left(V_{n}\right)_{n \in \omega}$ be a decreasing sequence of open sets containing $x$ such that $\left\{V_{n}\right\}_{n \in \omega}$ is a basis for the neighborhoods of $x$. Then $\mu\left(V_{n}\right)>0$ for all $n$ in $\omega$, since $x$ is in the support of $\mu$. For $n \in \omega$, choose a positive function $f_{n} \in C_{c}\left(V_{n}\right) \subseteq C_{c}(G)$ satisfying $f_{n}(x)=1$ and $\int f_{n} d \mu=1$.

Given $\xi \in L^{p}\left(\nu^{-1}\right)$ and $\eta \in L^{p^{\prime}}\left(\nu^{-1}\right)$, set

$$
\xi_{n}=\left(f_{n}^{\frac{1}{p}} \circ s\right) \xi \quad \text { and } \quad \eta_{n}=\left(f_{n}^{\frac{1}{p^{\prime}}} \circ s\right) \eta \text {. }
$$

Then

$$
\left\langle\operatorname{Ind}(y) f\left(\xi_{n}\right), \eta_{n}\right\rangle=f_{n}(y) \sum_{\rho \in G y} \sum_{\gamma \in r(\rho) G} f(\gamma) \xi\left(\gamma^{-1} \rho\right) \overline{\eta(\rho)}
$$

for all $y \in G^{0}$ and all $n \in \omega$. Fix $\varepsilon>0$. Since the map $y \mapsto\langle\operatorname{Ind}(y) f(\xi), \eta\rangle$ is continuous on $G^{0}$, there is $N \in \omega$ such that if $n \geq N$, then

$$
|\langle\operatorname{Ind}(y)(f) \xi, \eta\rangle-\langle\operatorname{Ind}(x)(f) \xi, \eta\rangle|<\varepsilon
$$

for every $y \in V_{n}$. For $n \geq N$, we have

$$
\begin{aligned}
& \left|\left\langle\operatorname{Ind}(\mu)(f) \xi_{n}, \eta_{n}\right\rangle-\langle\operatorname{Ind}(x)(f) \xi, \eta\rangle\right| \\
= & \int_{V_{n}} f_{n}(y)|\langle(\operatorname{Ind}(y)(f)-\operatorname{Ind}(x)(f)) \xi, \eta\rangle| d \mu(y)<\varepsilon .
\end{aligned}
$$


Therefore

$$
\begin{aligned}
& |\langle\operatorname{Ind}(x) f(\xi), \eta\rangle| \\
& =\lim _{n \rightarrow \infty}\left|\left\langle\operatorname{Ind}(\mu) f\left(\xi_{n}\right), \eta_{n}\right\rangle\right| \leq\|\operatorname{Ind}(\mu) f\| \lim _{n \rightarrow \infty}\left\|\xi_{n}\right\|\left\|\eta_{n}\right\| \\
& =\|\operatorname{Ind}(\mu) f\| \lim _{n \rightarrow \infty}\left(\sum_{\gamma \in G x}\left|\xi_{n}(\gamma)\right|^{p}\right)^{\frac{1}{p}} \lim _{n \rightarrow \infty}\left(\sum_{\gamma \in G x}\left|\eta_{n}(\gamma)\right|^{p^{\prime}}\right)^{\frac{1}{p^{\prime}}} \\
& =\|\operatorname{Ind}(\mu) f\|\|\xi\|\|\eta\| .
\end{aligned}
$$

This concludes the proof.

Corollary 6.16. The algebra $F_{\text {red }}^{p}(G)$ of $G$ is $p$-completely isometrically isomorphic to the enveloping $L^{p}$-operator algebra $F^{\mathcal{R}}\left(C_{c}(G)\right)$ with respect to the family of representations $\mathcal{R}=\left\{\operatorname{Ind}(x): x \in G^{0}\right\}$.

6.4. Amenable groupoids and their $L^{p}$-operator algebras. There are several equivalent characterizations of amenability for étale groupoids. By [1. Theorem 2.2.13], an étale groupoid is amenable if and only if has an approximate invariant mean.

Definition 6.17. An approximate invariant mean on $G$ is a sequence $\left(f_{n}\right)_{n \in \omega}$ of positive continuous compactly supported functions on $G$ such that

(1) $\sum_{\gamma \in x G} f_{n}(\gamma) \leq 1$ for every $n \in \omega$ and every $x \in G^{0}$,

(2) the sequence of functions $x \mapsto \sum_{\gamma \in x G} f_{n}(\gamma)$ converges to 1 uniformly on compact subsets of $G^{0}$, and

(3) the sequence of functions

$$
\gamma \rightarrow \sum_{\rho \in r(\gamma) G}\left|f_{n}\left(\rho^{-1} \gamma\right)-f_{n}(\rho)\right|
$$

converges to 0 uniformly on compact subsets of $G$.

Lemma 6.18. If $G$ is amenable and $m \geq 1$, then its amplification $G_{m}$ is amenable.

Proof. Let $\left(f_{n}\right)_{n \in \omega}$ be an approximate invariant mean for $G$. For $n \in \omega$, define $f_{n}^{(m)}: C_{c}\left(G_{m}\right) \rightarrow \mathbb{C}$ by $f_{n}^{(m)}(i, \gamma, j)=\frac{1}{m} f_{n}(\gamma)$ for $(i, \gamma, j) \in G_{m}$. It is not difficult to verify that $\left(f_{n}^{(m)}\right)_{n \in \omega}$ is an approximate invariant mean for $G_{m}$. We omit the details.

Definition 6.19. A pair of sequences $\left(g_{n}\right)_{n \in \omega}$ and $\left(h_{n}\right)_{n \in \omega}$ of positive functions in $C_{c}(G)$ is said to be an approximate invariant $p$-mean for $G$, if they satisfy the following:

(1) $\sum_{\gamma \in x G} g_{n}(\gamma)^{p} \leq 1$ and $\sum_{\gamma \in x G} h_{n}(\gamma)^{p^{\prime}} \leq 1$ for every $n \in \omega$ and every $x \in G^{0}$, 
(2) the sequence of functions $x \mapsto \sum_{\rho \in x G} g_{n}(\rho) h_{n}(\rho)$ converges to 1 uniformly on compact subsets of $G^{0}$, and

(3) the sequences of functions

$$
\gamma \mapsto \sum_{\rho \in r(\gamma) G}\left|g_{n}\left(\gamma^{-1} \rho\right)-g_{n}(\rho)\right|^{p}
$$

and

$$
\gamma \mapsto \sum_{\rho \in r(\gamma) G}\left|h_{n}\left(\gamma^{-1} \rho\right)-h_{n}(\rho)\right|^{p^{\prime}}
$$

converges to 0 uniformly on compact subsets of $G$.

It is not difficult to see that any amenable groupoid has an approximate invariant $p$-mean. Indeed, if $\left(f_{n}\right)_{n \in \omega}$ is any approximate invariant mean on $G$, then the sequences $\left(f_{n}^{1 / p}\right)_{n \in \omega}$ and $\left(f_{n}^{1 / p^{\prime}}\right)_{n \in \omega}$ define an approximate invariant $p$-mean on $G$.

Remark 6.20. It is easy to check that if $\left(g_{n}, h_{n}\right)_{n \in \omega}$ is an approximately invariant $p$-mean on $G$, then $\left(h_{n} * g_{n}\right)_{n \in \omega}$ converges to 1 uniformly on compact subsets of $G$.

The following theorem asserts that full and reduced $L^{p}$-operator algebras of amenable étale groupoids are canonically isometrically isomorphic.

Theorem 6.21. Suppose that $G$ is amenable. Then the canonical homomorphism $F^{p}(G) \rightarrow F_{\text {red }}^{p}(G)$ is a $p$-completely isometric isomorphism.

Proof. In view of Corollary 6.10 and Lemma 6.18, it is enough to show that the canonical $p$-completely contractive homomorphism from $F^{p}(G)$ to $F_{\text {red }}^{p}(G)$ is isometric. Let $\mu$ be a quasi-invariant measure on $G^{0}$, let $\lambda$ be a $\sigma$-finite Borel measure on a standard Borel space, and let $\lambda=\int \lambda_{x} d \mu(x)$ be the disintegration of $\lambda$ with respect to $\mu$. Let $T$ be a representation of $G$ on $\mathcal{Z}=\bigsqcup_{x \in G^{0}} L^{p}\left(\lambda_{x}\right)$, and let $\pi_{T}: C_{c}(G) \rightarrow B\left(L^{p}(\mu, \mathcal{Z})\right)$ be its integrated form. We want to show that $\left\|\pi_{T}(f)\right\| \leq\|f\|_{\text {red }}$.

Set $\mathcal{W}=\bigsqcup_{x \in G^{0}} \ell^{p}\left(G x, L^{p}\left(\lambda_{x}\right)\right)$ and let $\left(g_{n}, h_{n}\right)_{n \in \omega}$ be an approximate invariant $p$-mean for $G$. For $\xi \in L^{p}(\mu, \mathcal{Z})$ and $\eta \in L^{p^{\prime}}\left(\mu, \mathcal{Z}^{\prime}\right)$, define $\widehat{\xi}_{n} \in L^{p}(\mu, \mathcal{W})$ and $\widehat{\eta}_{n} \in L^{p^{\prime}}\left(\mu, \mathcal{W}^{\prime}\right)$ by

$$
\widehat{\xi}_{n, x}(\gamma)=D^{\frac{1}{p}}(\gamma) g_{n}(\gamma) T_{\gamma^{-1}} \xi_{r(\gamma)} \quad \text { and } \quad \widehat{\eta}_{n, x}(\gamma)=D^{\frac{1}{p^{\prime}}}(\gamma) h_{n}(\gamma) T_{\gamma^{-1}} \eta_{r(\gamma)} \text {. }
$$

Then

$$
\begin{aligned}
\int\left\|\widehat{\xi}_{n, x}\right\|^{p} d \mu(x) & =\int \sum_{\gamma \in G x} D(\gamma)\left|g_{n}(\gamma)\right|^{p}\left\|\xi_{r(\gamma)}\right\|^{p} d \mu(x) \\
& =\int D(\gamma)\left|g_{n}(\gamma)\right|^{p}\left\|\xi_{r(\gamma)}\right\|^{p} d \nu^{-1}(\gamma)
\end{aligned}
$$




$$
\begin{aligned}
& =\int\left|g_{n}(\gamma)\right|^{p}\left\|\xi_{r(\gamma)}\right\|^{p} d \nu(\gamma) \\
& =\int \sum_{\gamma \in x G}\left|g_{n}(\gamma)\right|^{p}\left\|\xi_{x}\right\|^{p} d \mu(x) \leq \int\left\|\xi_{x}\right\|^{p} d \mu(x) .
\end{aligned}
$$

This shows that $\widehat{\xi}_{n}$ belongs to $L^{p}(\mu, \mathcal{W})$ and that $\left\|\widehat{\xi}_{n}\right\| \leq\|\xi\|$. Similarly, $\widehat{\eta}_{n}$ belongs to $L^{p^{\prime}}\left(\mu, \mathcal{W}^{\prime}\right)$ and $\left\|\widehat{\eta}_{n}\right\| \leq\|\eta\|$.

Given $x \in G^{0}$, identify $\ell^{p}\left(G x, L^{p}\left(\lambda_{x}\right)\right)$ with $\ell^{p}(G x) \otimes L^{p}\left(\lambda_{x}\right)$ and consider the representation $\operatorname{Ind}(x) \otimes 1: C_{c}(G) \rightarrow B\left(\ell^{p}\left(G x, L^{p}\left(\lambda_{x}\right)\right)\right)$, which for $f \in$ $C_{c}(G)$ is given by is given byQTOallowdisplaybreaks

$$
\langle(\operatorname{Ind}(x) \otimes 1)(f) v, w\rangle=\sum_{\gamma \in G x} \sum_{\rho \in r(\gamma) G} f(\rho)\left\langle v\left(\rho^{-1} \gamma\right), w(\gamma)\right\rangle
$$

for $v \in \ell^{p}\left(G x, L^{p}\left(\lambda_{x}\right)\right)$ and $w \in \ell^{p^{\prime}}\left(G x, L^{p^{\prime}}\left(\lambda_{x}\right)\right)$.

Set $\pi=\int(\operatorname{Ind}(x) \otimes 1) d \mu(x): C_{c}(G) \rightarrow B\left(L^{p}(\mu, \mathcal{W})\right)$. Fix $v \in L^{p}(\mu, \mathcal{W})$ and $w \in L^{p^{\prime}}\left(\nu, \mathcal{W}^{\prime}\right)$ with $\|v\|,\|w\| \leq 1$. Then

$$
\begin{aligned}
\langle\pi(f) v, w\rangle & =\int\left\langle(\operatorname{Ind}(x) \otimes 1)(f) v_{x}, w_{x}\right\rangle d \mu(x) \\
& =\int \sum_{\gamma \in G x} \sum_{\rho \in r(\gamma) G} f(\rho)\left\langle v_{x}\left(\rho^{-1} \gamma\right), w_{x}(\gamma)\right\rangle d \mu(x)
\end{aligned}
$$

and hence

$$
\begin{aligned}
|\langle\pi(f) v, w\rangle| & \leq \int\left|\left\langle(\operatorname{Ind}(x) \otimes 1)(f) v_{x}, w_{x}\right\rangle\right| d \mu \\
& \leq \int\|(\operatorname{Ind}(x) \otimes 1)(f)\|\left\|v_{x}\right\|\left\|w_{x}\right\| d \mu \\
& \leq \int\|\operatorname{Ind}(x)(f)\|\left\|v_{x}\right\|\left\|w_{x}\right\| d \mu \\
& \leq\|f\|_{\text {red }} \int\left\|v_{x}\right\|\left\|w_{x}\right\| d \mu(x) \leq\|f\|_{\text {red }} .
\end{aligned}
$$

We conclude that $\|\pi(f)\| \leq\|f\|_{\text {red }}$ for all $f \in C_{c}(G)$. In particular, for $v=\widehat{\xi}_{n}$ and $w=\widehat{\eta}_{n}$, one gets

$$
\begin{aligned}
& \left\langle\pi(f) \widehat{\xi}_{n}, \widehat{\eta}_{n}\right\rangle \\
& =\int \sum_{\gamma \in G x} \sum_{\rho \in s(\gamma) G} f(\rho)\left\langle\widehat{\xi}_{n, x}\left(\rho^{-1} \gamma\right), \widehat{\eta}_{n, x}(\gamma)\right\rangle d \mu \\
& =\int \sum_{\gamma \in x G} \sum_{\rho \in x G} f(\rho) D^{-\frac{1}{p}}(\rho) g_{n}\left(\rho^{-1} \gamma\right) h_{n}(\gamma)\left\langle T_{\rho} \xi_{s(\rho)}, \eta_{x}\right\rangle d \mu(x)
\end{aligned}
$$




$$
\begin{aligned}
& =\int \sum_{\rho \in x G}\left(\sum_{\gamma \in x G} g_{n}\left(\rho^{-1} \gamma\right) h_{n}(\gamma)\right) f(\rho) D^{-\frac{1}{p}}(\rho)\left\langle T_{\rho} \xi_{s(\rho)}, \eta_{x}\right\rangle d \mu(x) \\
& =\int \sum_{\rho \in x G}\left(\sum_{\gamma \in x G} h_{n}(\gamma) g_{n}^{*}\left(\gamma^{-1} \rho\right)\right) f(\rho) D^{-\frac{1}{p}}(\rho)\left\langle T_{\rho} \xi_{s(\rho)}, \eta_{x}\right\rangle d \mu(x) \\
& =\int \sum_{\rho \in x G}\left(h_{n} * g_{n}\right)(\rho) f(\rho) D^{-\frac{1}{p}}(\rho)\left\langle T_{\rho} \xi_{s(\rho)}, \eta_{x}\right\rangle d \mu(x)
\end{aligned}
$$

and thus

$$
\begin{aligned}
& \lim _{n \rightarrow \infty}\left\langle\pi(f) \widehat{\xi}_{n}, \widehat{\eta}_{n}\right\rangle \\
& =\lim _{n \rightarrow \infty} \int \sum_{\rho \in x G}\left(h_{n} * g_{n}\right)(\rho) f(\rho) D^{-\frac{1}{p}}(\rho)\left\langle T_{\rho} \xi_{s(\rho)}, \eta_{x}\right\rangle d \mu(x) \\
& =\int \sum_{\rho \in x G} f(\rho) D^{-\frac{1}{p}}(\rho)\left\langle T_{\rho} \xi_{s(\rho)}, \eta_{x}\right\rangle d \mu(x)=\left\langle\pi_{T}(f) \xi, \eta\right\rangle .
\end{aligned}
$$

We conclude that

$$
\left\|\pi_{T}(f)\right\| \leq \lim _{n \rightarrow \infty}\left|\left\langle\pi(f) \widehat{\xi}_{n}, \widehat{\eta}_{n}\right\rangle\right| \leq\|\pi(f)\| \leq\|f\|_{\text {red }},
$$

as desired.

\section{Examples: analogs of Cuntz algebras and AF-Algebras}

Throughout this section, we let $p \in(1, \infty)$.

7.1. The Cuntz $L^{p}$-operator algebras. Fix $d \in \omega$ with $d \geq 2$. The following is [27, Definition 1.1] and [27, Definition 7.4 (2)]. Algebra representations of complex unital algebras are always assumed to be unital.

Definition 7.1. Define the Leavitt algebra $L_{d}$ to be the universal (complex) algebra with generators $s_{0}, \ldots, s_{d-1}, s_{0}^{*}, \ldots, s_{d-1}^{*}$, subject to the relations

(1) $s_{j}^{*} s_{k}=\delta_{j, k}$ for $j, k \in d$; and

(2) $\sum_{j \in d} s_{j} s_{j}^{*}=1$.

If $\lambda$ is a $\sigma$-finite Borel measure on a standard Borel space, a spatial representation of $L_{d}$ on $L^{p}(\lambda)$ is an algebra homomorphism $\rho: L_{d} \rightarrow \mathcal{S}\left(L^{p}(\lambda)\right)$ such that for $j \in d$, the operators $\rho\left(s_{j}\right)$ and $\rho\left(s_{j}^{*}\right)$ are mutually inverse spatial partial isometries, i.e. $\rho\left(s_{j}^{*}\right)=\rho\left(s_{j}\right)^{*}$.

It is a consequence of a fundamental result of J. Cuntz from [8] that any two ${ }^{*}$-representations of $L_{d}$ on a Hilbert space induce the same norm on $L_{d}$. The corresponding completion is the Cuntz $\mathrm{C}^{*}$-algebra $\mathcal{O}_{d}$.

Cuntz's result was later generalized by N.C. Phillps in 27] to spatial representations of $L_{d}$ on $L^{p}$-spaces. Theorem 8.7 of [27] asserts that any 
two spatial $L^{p}$-representations of $L_{d}$ induce the same norm on it. The corresponding completion is the Cuntz $L^{p}$-operator algebra $\mathcal{O}_{d}^{p}$; see [27, Definition 8.8]. We now to explain how one can realize $\mathcal{O}_{d}^{p}$ as a groupoid $L^{p}$-operator algebra.

Denote by $d^{\omega}$ the space of infinite sequences of elements of $d$, endowed with the product topology. (Recall that $d$ is identified with the set $\{0,1, \ldots, d-1\}$ of its predecessors.) Denote by $d^{<\omega}$ the space of (possibly empty) finite sequences of elements of $d$. The length of an element $a$ of $d^{<\omega}$ is denoted by $\operatorname{lh}(a)$. For $a \in d^{<\omega}$ and $x \in d^{\omega}$, define $a^{\curvearrowright} x \in d^{\omega}$ to be the concatenation of $a$ and $x$. For $a \in d^{<\omega}$, denote by $[a]$ the set of elements of $d^{\omega}$ having $a$ as initial segment, i.e.

$$
[a]=\left\{a^{\wedge} x: x \in d^{\omega}\right\} .
$$

Clearly $\left\{[a]: a \in d^{<\omega}\right\}$ is a clopen basis for $d^{\omega}$.

Definition 7.2. The Cuntz inverse semigroup $\Sigma_{d}$ is the inverse semigroup generated by a zero 0 , a unit 1 , and elements $s_{j}$ for $j \in d$, satisfying $s_{j}^{*} s_{k}=0$ whenever $j \neq k$.

Set $s_{\varnothing}=1$ and $s_{a}=s_{a_{0}} \cdots s_{a_{l h(a)-1}} \in \Sigma_{d}$ for $a \in d^{<\omega} \backslash\{\varnothing\}$. Every element of $\Sigma_{d}$ can be written uniquely as $s_{a} s_{b}^{*}$ for some $a, b \in d^{<\omega}$.

Remark 7.3. The nonzero idempotents $E\left(\Sigma_{d}\right)$ of $\Sigma_{d}$ are precisely the elements of the form $s_{a} s_{a}^{*}$ for $a \in d^{<\omega}$. Moreover, the function $d^{<\omega} \cup\{0\} \rightarrow$ $E(\Sigma)$ given by $a \mapsto s_{a} s_{a}^{*}$ and $0 \mapsto 0$, is a semilattice map, where $d^{<\omega}$ has its (downward) tree ordering defined by $a \leq b$ if and only if $b$ is an initial segment of $a$, and 0 is a least element of $d^{<\omega} \cup\{0\}$.

Observe that if $a, b \in d^{<\omega}$, then $a b=0$ if and only if $a(j) \neq b(j)$ for some $j \in \min \{\operatorname{lh}(a), \operatorname{lh}(b)\}$

Lemma 7.4. Let $\mathcal{B}$ be a Boolean algebra and let $\beta: d^{<\omega} \rightarrow \mathcal{B}$ be a representation. Then $\beta$ is tight if and only if $\beta(\varnothing)=1$ and

$$
\beta(a) \leq \bigvee_{j \in d} \beta\left(a^{\wedge} j\right)
$$

for every $a \in d^{<\omega}$.

Proof. Suppose that $\beta$ is tight. Since 1 is a cover of $E^{\varnothing, \varnothing}$, we have $\beta(\varnothing)=1$. Similarly, $\left\{a^{\wedge} j: j \in d\right\}$ is a cover of $E^{\{a\}, \varnothing}$ and thus $\beta(a) \leq \bigvee_{j \in d} \beta\left(a^{\frown} j\right)$. Let us now show the "if" implication. By [10, Proposition 11.8], it is enough to show that for every $a \in d^{<\omega}$ and every finite cover $Z$ of $\{a\}$, one has $\beta(a) \leq$ $\bigvee_{z \in Z} \beta(z)$. That this is true follows from the hypotheses, using induction on the maximum length of elements of $Z$. 
Lemma 7.5. Let $\lambda$ be a $\sigma$-finite Borel measure on a standard Borel space, and $\rho$ be a representation of $\Sigma_{d}$ on $L^{p}(\lambda)$. Then $\rho$ is tight if and only if

$$
\sum_{j \in d} \rho\left(s_{j} s_{j}^{*}\right)=\rho(1)=1 .
$$

Proof. Suppose that $\rho$ is tight. Then $\left.\rho\right|_{E(\Sigma)}$ is tight and therefore

$$
1=\rho(1)=\bigvee_{j \in d} \rho\left(s_{j} s_{j}^{*}\right)=\sum_{j \in d} \rho\left(s_{j} s_{j}^{*}\right)
$$

by Lemma 7.4. Conversely, given $a \in d^{<\omega}$, we have

$$
\begin{aligned}
\sum_{j \in d} \rho\left(s_{a^{\wedge} j} s_{a^{\wedge} j}^{*}\right) & =\sum_{j \in d} \rho\left(s_{a} s_{j} s_{j}^{*} s_{a}^{*}\right)=\sum_{j \in d} \rho\left(s_{a}\right) \rho\left(s_{j} s_{j}^{*}\right) \rho\left(s_{a}^{*}\right) \\
& =\rho\left(s_{a}\right)\left(\sum_{j \in d} \rho\left(s_{j} s_{j}^{*}\right)\right) \rho\left(s_{a}^{*}\right)=\rho\left(s_{a}\right) \rho(1) \rho\left(s_{a}^{*}\right) \\
& =\rho\left(s_{a} s_{a}^{*}\right),
\end{aligned}
$$

which shows that $\rho$ is tight, concluding the proof.

Proposition 7.6. The algebra $F_{\text {tight }}^{p}\left(\Sigma_{d}\right)$ is $p$-completely isometric isomorphic to $\mathcal{O}_{d}^{p}$.

Proof. Observe that the Leavitt algebra $L_{d}$ (see Definition 7.1) is isomorphic to the quotient of $\mathbb{C} \Sigma_{d}$ by the ideal generated by the elements $\delta_{1}-\sum_{j \in d} \delta_{s_{j} s_{j}^{*}}$ and $\delta_{0}$. (Here, $\delta_{s}$ denotes the canonical element in $\mathbb{C} \Sigma_{d}$ corresponding to $s \in \Sigma_{d}$.) By Lemma 7.5, tight representations of $\Sigma_{d}$ correspond precisely to spatial representations of the Leavitt algebra $L_{d}$ as defined in 27, Definition 7.4]. The result then follows.

It is well known that $\Sigma_{d}$ an inverse semigroup of compact open slices of an ample groupoid $\mathcal{G}_{d}$. We now proceed to define $\mathcal{G}_{d}$. Let $T: d^{\omega} \rightarrow d^{\omega}$ denote the unilateral shift on $d^{\omega}$, and observe that $T$ is one-to-one on $[a]$ whenever $\ln (a) \geq 1$. Denote by $\mathcal{G}_{d}$ the groupoid

$$
\mathcal{G}_{d}=\left\{(x, m-n, y): x, y \in d^{\omega}, m, n \in \mathbb{N}, T^{m} x=T^{n} y\right\},
$$

with operations defined by

$$
\begin{gathered}
s(x, m-n, y)=x \quad, \quad r(x, m-n, y)=y \\
(x, m-n, y)(y, k-r, z)=(x, m-n+k-r, z) \\
(x, k, y)^{-1}=(y,-k, x) .
\end{gathered}
$$

For $a$ and $b$ in $d^{<\omega}$, set

$$
[a, b]=\left\{\left(a^{\wedge} x, \ln (a)-\ln (b), b^{\curvearrowright} x\right): x \in d^{\omega}\right\} \subseteq \mathcal{G}_{d} .
$$

The collection $\left\{[a, b]: a, b \in d^{<\omega}\right\}$ is a basis of clopen slices for $\mathcal{G}_{d}$, and $\mathcal{G}_{d}$ is therefore ample. 
Theorem 7.7. Let $d \geq 2$ be a positive integer, and let $\mathcal{G}_{d}$ denote the corresponding Cuntz groupoid. Then $F^{p}\left(\mathcal{G}_{d}\right)$ is canonically $p$-completely isometrically isomorphic to $\mathcal{O}_{d}^{p}$.

Proof. It is easy to check that the function $s_{a} s_{b}^{*} \mapsto[a, b]$ defines an injective homomorphism from $\Sigma_{d}$ to the inverse semigroup of compact open slices of $\mathcal{G}_{d}$. It is well known that $\mathcal{G}_{d}$ is amenable; see [38, Exercise 4.1.7]. It follows from Theorem 6.21, Corollary 6.11, and Proposition 7.6, that there are canonical $p$-completely isometric isomorphisms

$$
F_{\text {red }}^{p}\left(\mathcal{G}_{d}\right) \cong F^{p}\left(\mathcal{G}_{d}\right) \cong F_{\text {tight }}^{p}\left(\Sigma_{d}\right) \cong \mathcal{O}_{d}^{p}
$$

7.2. Analogs of AF-algebras on $L^{p}$-spaces. In this subsection, we show how one can use the machinery developed in the previous sections to construct those $L^{p}$-analogs of $\mathrm{AF}$-algebras that look like $\mathrm{C}^{*}$-algebras, and which are called "spatial" in 31.

Fix $n \in \mathbb{N}$. The algebra $M_{n}(\mathbb{C})$ of $n \times n$ matrices with complex coefficients can be (algebraically) identified with $B\left(\ell^{p}(n)\right)$. This identification turns $M_{n}(\mathbb{C})$ into an $L^{p}$-operator algebra that we will denote - consistently with [27]-by $M_{n}^{p}$. It is not difficult to verify that $M_{n}^{p}$ can be realized as a groupoid $L^{p}$-operator algebra, and we proceed to outline the argument.

Denote by $T_{n}$ the principal groupoid determined by the trivial equivalence relation on $n$. It is well-known (see [37, page 121]) that $T_{n}$ is amenable. Moreover, the inverse semigroup $\Sigma_{\mathcal{K}}\left(T_{n}\right)$ of compact open slices of $T_{n}$, is the inverse semigroup generated by a zero element 0 , a unit 1 , and elements $e_{j k}$ for $j, k \in n$, subject to the relations $e_{j k}^{*} e_{\ell m}=\delta_{k \ell} e_{j m}$ for $j, k, \ell, m \in n$. It is not difficult to verify, using Lemma 4.19, that a tight $L^{p}$-representation $\rho$ of $\Sigma_{\mathcal{K}}(T)$ satisfies

$$
1=\rho(1)=\sum_{j \in n} \rho\left(e_{j j}\right)
$$

It thus follows from [27, Theorem 7.2] that the map from $M_{n}^{p}$ to the range of $\rho$, defined by assigning $\rho\left(e_{j k}\right)$ to the $j k$-th matrix unit in $M_{n}^{p}$, is isometric. We conclude that $F^{p}\left(T_{n}\right)$ is isometrically isomorphic to $M_{n}^{p}$. Reasoning in the same way at the level of amplifications shows that $F^{p}\left(T_{n}\right)$ and $M_{n}^{p}$ are in fact $p$-completely isometrically isomorphic.

If $k \in \mathbb{N}$ and $\mathbf{n}=\left(n_{0}, \ldots, n_{k-1}\right)$ is a $k$-tuple of natural numbers, then the Banach algebra $M_{n_{0}}^{p} \oplus \cdots \oplus M_{n_{k-1}}^{p}$ acts naturally on the $L^{p}$-direct $\operatorname{sum} \ell^{p}\left(n_{0}\right) \oplus \cdots \oplus \ell^{p}\left(n_{k-1}\right) \cong \ell^{p}\left(n_{0}+\cdots+n_{k-1}\right)$. The Banach algebra $M_{n_{0}}^{p} \oplus \cdots \oplus M_{n_{k-1}}^{p}$ can also be realized as groupoid $L^{p}$-operator algebra by considering the disjoint union of the groupoids $T_{n_{0}}, T_{n_{1}}, \ldots, T_{n_{k-1}}$.

Here is the definition of spatial $L^{p}$-operator AF-algebras 
Definition 7.8. A separable Banach algebra $A$ is said to be a spatial $L^{p}$-operator AF-algebra if there exists a direct system $\left(A_{n}, \varphi_{n}\right)_{n \in \omega}$ of $L^{p}$ operator algebras $A_{n}$ which are isometrically isomorphic to algebras of the form $M_{n_{0}}^{p} \oplus \cdots \oplus M_{n_{k}}^{p}$, with isometric connecting maps $\varphi_{n}: A_{n} \rightarrow A_{n+1}$, and such that $A$ is isometrically isomorphic to the direct $\operatorname{limit} \underset{\lim }{\longrightarrow}\left(A_{n}, \varphi_{n}\right)_{n \in \omega}$.

Banach algebras as in the definition above, as well as more general direct limits of semisimple finite-dimensional $L^{p}$-operator algebras, will be studied in 31 .

In the rest of this subsection, we will show that spatial $L^{p}$-operator $\mathrm{AF}$ algebras can be realized as groupoid $L^{p}$-operator algebras.

For simplicity, we will start by observing that spatial $L^{p}$-operator UHFalgebras are groupoid $L^{p}$-operator algebras. Spatial $L^{p}$-operator UHF-algebras are the spatial $L^{p}$-operator AF-algebras where the building blocks $A_{n}$ appearing in the definition are all full matrix algebras $M_{d_{n}}^{p}$ for some $d_{n} \in \omega$. These have been defined and studied in [29].

Let $d=\left(d_{n}\right)_{n \in \omega}$ be a sequence of positive integers. Denote by $A_{d}^{p}$ the corresponding $L^{p}$-operator UHF-algebra defined as above; see also [30, Definition 3.9]. In the following we will show that $A_{d}^{p}$ is the enveloping algebra of a natural groupoid associated with the sequence $d$. Define $Z_{d}=\prod_{j \in n} d_{j}$, and consider the groupoid

$$
G_{d}=\left\{\left(\alpha^{\frown} x, \beta^{\frown} x\right): \alpha, \beta \in \prod_{j \in n} d_{j}, x \in \prod_{j \geq n} d_{j}, n \in \omega\right\}
$$

having $Z_{d}$ as set of objects. (Here we identify $x \in Z_{d}$ with the pair $(x, x) \in$ $G_{d}$.) The operations are defined by

$$
\begin{aligned}
& s\left(\alpha^{\frown} x, \beta^{\frown} x\right)=\beta^{\frown} x, \\
& \left(\alpha^{\wedge} x, \beta^{\frown} x\right)^{-1}=\left(\beta^{\wedge} x, \alpha^{\wedge} x\right), \text { and } \\
& \left(\alpha^{\frown} x, \beta^{\frown} x\right)\left(\gamma^{\wedge} y, \delta^{\frown} y\right)=\left(\alpha^{\frown} x, \delta^{\frown} y\right) \text { whenever } \beta^{\urcorner} x=\gamma^{\frown} y \text {. }
\end{aligned}
$$

It is well-known that $G_{d}$ is amenable; see [38, Chapter III, Remark 1.2].

Given $k \in \omega$ and given $\alpha$ and $\beta$ in $\prod_{j \in k} d_{j}$, define

$$
U_{\alpha \beta}=\left\{\left(\alpha^{\wedge} x, \beta^{\wedge} x\right) \in G_{d}: x \in \prod_{j \geq k} d_{j}\right\} .
$$

Then

$$
\left\{U_{\alpha \beta}: \alpha, \beta \in \prod_{j \in k} d_{j}, k \in \omega\right\}
$$


is a basis of compact open slices for an ample groupoid topology on $G_{d}$.

Fix $k \in \omega$ and consider the compact groupoid

$$
G_{d}^{k}=\bigcup\left\{U_{\alpha, \beta}: \alpha, \beta \in \prod_{j \in k} d_{j}\right\} .
$$

The groupoid $G_{d}$ can be seen as the topological direct limit of the system $\left(G_{d}^{k}\right)_{k \in \omega}$. It is clear that, if $n=d_{0} \cdots d_{k-1}$, then $G_{d}^{k}$ is isomorphic to the groupoid $T_{n}$ defined previously. Therefore $F^{p}\left(G_{d}^{k}\right)$ is isometrically isomorphic to $M_{d_{0} \cdots d_{k-1}}^{p}$.

For $k \in \mathbb{N}$, identify $C\left(G_{d}^{k}\right)$ with a ${ }^{*}$-subalgebra of $C_{c}\left(G_{d}\right)$, by setting $f \in C\left(G_{d}^{k}\right)$ to be 0 outside $G_{d}^{k}$. For $k<n$, we claim that the inclusion map from $C\left(G_{d}^{k}\right)$ to $C\left(G_{d}^{n}\right)$ induces an isometric embedding

$$
\varphi_{n}: F^{p}\left(G_{d}^{k}\right) \rightarrow F^{p}\left(G_{d}^{n}\right) .
$$

This can be easily verified by direct computation, after noticing that $G_{d}^{k}$ and $G_{d}^{n}$ are amenable, and hence the full and reduced norms on $C\left(G_{d}^{k}\right)$ and $C\left(G_{d}^{n}\right)$ coincide. One then obtains a direct system $\left(F^{p}\left(G_{d}^{k}\right), \varphi_{n}\right)_{n \in \mathbb{N}}$ with isometric connecting maps whose limit is $F^{p}(G)$. Since $F^{p}\left(G_{d}^{k}\right) \cong M_{d_{0} \cdots d_{k-1}}^{p}$ as observed above, we conclude that $F^{p}\left(G_{d}\right) \cong A_{d}^{p}$.

We now turn to spatial AF-algebras. As in the $\mathrm{C}^{*}$-algebra case, there is a natural correspondence between $L^{p}$-operator AF-algebras and Bratteli diagrams. (For the definition of Bratteli diagrams, see [39, Subsection 7.2.3].) Let $(E, V)$ be a Bratteli diagram, and $A^{(E, V)}$ be the associated $L^{p}$-operator AF-algebra. In the following, we will explain how to realize $A^{(E, V)}$ as a groupoid $L^{p}$-operator algebra.

Denote by $X$ the set of all infinite paths in $(E, V)$. Then $X$ is a compact zero-dimensional space. Denote by $G^{(E, V)}$ the tail equivalence relation on $X$, regarded as a principal groupoid having $X$ as set of objects. It is well known that $G^{(E, V)}$ is amenable; see [38, Chapter III, Remark 1.2]. If $\alpha, \beta$ are finite paths of the same length and with the same endpoints, define $U_{\alpha \beta}$ to be the set of elements of $G^{(E, V)}$ of the form $\left(\alpha^{\wedge} x, \beta^{\wedge} x\right)$. The collection of all the sets $U_{\alpha \beta}$ is a basis for an ample groupoid topology on $G^{(E, V)}$. For $k \in \omega$, let $G_{k}^{(E, V)}$ be the union of $U_{\alpha \beta}$ over all finite paths $\alpha, \beta$ as before that moreover have length at most $k$. Then $G_{n}^{(E, V)}$ is a compact groupoid and $G$ is the topological direct limit of $\left(G_{k}^{(E, V)}\right)_{k \in \omega}$.

Fix $k \in \omega$. Denote by $l$ the cardinality of the $k$-th vertex set $V_{k}$. Denote by $n_{0}, \ldots, n_{l-1}$ the multiplicities of the vertices in $V_{k}$. (The multiplicity of a vertex in a Bratteli diagram is defined in the usual way by recursion.) Set $\mathbf{n}=\left(n_{0}, \ldots, n_{l-1}\right)$, and observe that $G_{k}^{(E, V)}$ is isomorphic to the groupoid $T_{\mathbf{n}}$ as defined above. In particular

$$
F^{p}\left(G_{n}^{(E, V)}\right) \cong M_{n_{0}}^{p} \oplus \cdots \oplus M_{n_{l-1}}^{p} .
$$


As before, one can show that the direct system $\left(F^{p}\left(G_{n}^{(E, V)}\right)\right)_{n \in \omega}$ has isometric connecting maps, and that the inductive limit is $F^{p}\left(G^{(E, V)}\right)$. This concludes the proof that $A^{(E, V)}$ is $p$-completely isometrically isomorphic to $F^{p}\left(G^{(E, V)}\right)$. In particular, this shows that $A^{(E, V)}$ is indeed an $L^{p}$-operator algebra.

\section{Concluding Remarks AND OUtLOOK}

It is not difficult to see that the class of $L^{p}$-operator algebras is closedwithin the class of all matricially normed Banach algebras - under taking subalgebras and ultraproducts. As observed by Ilijas Farah and Chris Phillips, this observation, together with a general result from logic for metric structures, implies that the class of $L^{p}$-operator algebras is -in modeltheoretic jargon-universally axiomatizable. This means that $L^{p}$-operator algebras can be characterized as those matricially normed Banach algebras satisfying certain expressions only involving

- the algebra operations,

- the matrix norms,

- continuous functions from $\mathbb{R}^{n}$ to $\mathbb{R}$, and

- suprema over balls of matrix amplifications.

Determining what these expressions are seems to be, in our opinion, an important problem in the theory of algebras of operators on $L^{p}$-spaces.

Problem 8.1. Find an explicit intrinsic characterization of $L^{p}$-operator algebras within the class of matricially normed Banach algebras.

An explicit characterization of algebras acting on subspaces of quotients of $L^{p}$-spaces was provided by Le Merdy in [20]. These are precisely the matricially normed Banach algebras that are moreover $p$-operator spaces in the terminology of [9], and such that multiplication is $p$-completely contractive. Similar results have been obtained by Junge for algebras of operators on subspaces of $L^{p}$-spaces; see [16, Corollary 1.5.2.2].

The problem of finding an explicit intrinsic characterization of $L^{p}$-operator algebras is tightly related to the problem whether the class of $L^{p}$-operator algebras is closed by taking quotients.

Problem 8.2. Is the quotient an $L^{p}$-operator algebra by a closed two-sided ideal an $L^{p}$-operator algebra?

This problem was affirmatively solved for algebras of operators on subspaces of quotients of $L^{p}$-spaces by Le Merdy in [20, as a corollary of his intrinsic characterization. A similar result for algebras of operators on subspaces of $L^{p}$-spaces is contained in [16, Corollary 1.5.2.3].

When $p=1$, Problem 8.2 was shown to have a negative answer by the first author and Hannes Thiel in [13]. 
Showing that the class of $L^{p}$-operator algebras is closed by taking quotients would significantly simplify the problem of determining simplicity of $L^{p}$-operator algebras. In fact, in order to show that an $L^{p}$-operator algebra is simple, it would then be enough to prove that every nonzero representation on an $L^{p}$-space is injective. This would make it possible to adapt methods from the theory of $\mathrm{C}^{*}$-algebras. For example, it is shown in [6, Theorem 5.1] that the reduced $\mathrm{C}^{*}$-algebra of an étale groupoid is simple if and only the groupoid is minimal and topologically principal. (A groupoid is called minimal if it has no nontrivial invariant open set of objects, and topologically principal if the set of objects with trivial isotropy group is dense.) We believe that the same should be true for the reduced $L^{p}$-operator algebras of étale groupoids. This has been shown for $L^{p}$-analogs of UHF-algebras and Cuntz algebras in [30] by seemingly ad hoc methods.

Problem 8.3. Is $F_{r e d}^{p}(G)$ simple whenever $G$ is a minimal and topologically principal étale groupoid?

A potential application of groupoids to the theory of $L^{p}$-operator algebras comes from the technique of Putnam subalgebras. Let $X$ be a compact metric space and let $h: X \rightarrow X$ be a homeomorphism. Denote by $u$ the canonical unitary in the $\mathrm{C}^{*}$-crossed product $C^{*}(\mathbb{Z}, X, h)$ implementing $h$. If $Y$ is a closed subset of $X$, then the corresponding Putnam subalgebra $C^{*}(\mathbb{Z}, X, h)_{Y}$ is the $\mathrm{C}^{*}$-subalgebra of $C^{*}(\mathbb{Z}, X, h)$ generated by $C(X)$ and $u C_{0}(X \backslash Y)$. It is known that $C^{*}(\mathbb{Z}, X, h)_{Y}$ can be described as the enveloping $\mathrm{C}^{*}$-algebra of a suitable étale groupoid.

In the context of $\mathrm{C}^{*}$-algebras, Putnam subalgebras are fundamental in the study of transformation group $\mathrm{C}^{*}$-algebras of minimal homeomorphisms. For example, Putnam showed in [34, Theorem 3.13] that if $h$ is a minimal homeomorphism of the Cantor space $X$, and $Y$ is a nonempty clopen subset of $X$, then $C^{*}(\mathbb{Z}, X, h)_{Y}$ is an AF-algebra. This is then used in 34 to prove that the crossed product $C^{*}(\mathbb{Z}, X, h)_{Y}$ is a simple AT-algebra of real rank zero. Similarly, Putnam subalgebras were used by Huaxin Lin and Chris Phillips in [21] to show that, under a suitable assumption on $K$-theory, the crossed product of a finite-dimensional compact metric space by a minimal homeomorphism is a simple unital $\mathrm{C}^{*}$-algebra with tracial rank zero.

Considering the groupoid description of Putnam subalgebras provides a natural application of our constructions to the theory of $L^{p}$-crossed products introduced in [28]. It is conceivable that with the aid of groupoid $L^{p}$-operator algebras, Putnam subalgebras could be used to obtain generalizations of the above mentioned results to $L^{p}$-crossed products.

\section{REFERENCES}

[1] Claire Anantharaman-Delaroche and Jean Renault, Amenable groupoids, Monographies de L'Enseignement Mathématique, vol. 36, L'Enseignement Mathématique, Geneva, 2000.

[2] Stefan Banach, Théorie des opérations linéaires, Éditions Jacques Gabay, Sceaux, 1993, Reprint of the 1932 original. 
[3] Earl Berkson, Hermitian projections and orthogonality in Banach spaces, Proceedings of the London Mathematical Society 24 (1972), no. 3, 101-118.

[4] Bruce Blackadar, Operator algebras, Encyclopaedia of Mathematical Sciences, vol. 122, Springer-Verlag, Berlin, 2006.

[5] David P. Blecher and Christian Le Merdy, Operator Algebras and their Modules, London Mathematical Society Monographs, vol. 30, Oxford University Press, Oxford, 2004.

[6] Jonathan Brown, Lisa Orloff Clark, Cynthia Farthing, and Aidan Sims, Simplicity of algebras associated to étale groupoids, Semigroup Forum 88 (2014), no. 2, 433-452.

[7] Neal L. Carothers, A Short Course on Banach Space Theory, London Mathematical Society Student Texts, vol. 64, Cambridge University Press, Cambridge, 2005.

[8] Joachim Cuntz, Simple $C^{*}$-algebras generated by isometries, Communications in Mathematical Physics 57 (1977), no. 2, 173-185.

[9] Matthew Daws, $p$-Operator spaces and Figà-Talamanca-Herz algebras, Journal of Operator Theory 63 (2010), no. 1, 47-83.

[10] Ruy Exel, Inverse semigroups and combinatorial $C^{*}$-algebras, Bulletin of the Brazilian Mathematical Society, New Series 39 (2008), no. 2, 191-313.

[11] Richard J. Fleming and James E. Jamison, Isometries on Banach spaces: function spaces, Monographs and Surveys in Pure and Applied Mathematics, vol. 129, Chapman \& Hall/CRC, Boca Raton, FL, 2003.

[12] Eusebio Gardella and Hannes Thiel, Group algebras acting on $L^{p}$-spaces, in preparation.

[13] Banach algebras generated by an invertible isometry of an $L^{p}$-space, arXiv:1405.5589 (2014).

[14] Siegfried Graf and R. Daniel Mauldin, A classification of disintegrations of measures, Measure and measurable dynamics, Contemp. Math., vol. 94, Amer. Math. Soc., Providence, RI, 1989, pp. 147-158.

[15] Peter Hahn, Haar measure for measure groupoids, Transactions of the American Mathematical Society 242 (1978), 1-33.

[16] Marius Junge, Factorization theory for spaces of operators, Habilitationsschrift (1996).

[17] Alexander S. Kechris, Classical Descriptive Set Theory, Graduate Texts in Mathematics, vol. 156, Springer-Verlag, New York, 1995.

[18] Alex Kumjian, David Pask, Iain Raeburn, and Jean Renault, Graphs, groupoids, and Cuntz-Krieger algebras, Journal of Functional Analysis 144 (1997), no. 2, 505-541.

[19] John Lamperti, On the isometries of certain function-spaces, Pacific Journal of Mathematics 8 (1958), no. 3, 459-466.

[20] Christian Le Merdy, Representation of a quotient of a subalgebra of $B(X)$, Mathematical Proceedings of the Cambridge Philosophical Society 119 (1996), no. 01, 83-90.

[21] Huaxin Lin and N. Christopher Phillips, Crossed products by minimal homeomorphisms, Journal für die Reine und Angewandte Mathematik 641 (2010), 95-122.

[22] Günter Lumer, Semi-inner-product spaces, Transactions of the American Mathematical Society 100 (1961), no. 1, 29-43.

[23] Martino Lupini, Polish groupoids and functorial complexity, arXiv:1407.6671 (2014).

[24] George W. Mackey, Ergodic theory, group theory, and differential geometry, Proceedings of the National Academy of Sciences of the United States of America 50 (1963), $1184-1191$

[25] Christian Le Merdy, Factorization of p-completely bounded multilinear maps, Pacific Journal of Mathematics 172 (1996), no. 1, 187-213.

[26] Alan L. T. Paterson, Groupoids, Inverse Semigroups, and their Operator Algebras, Progress in Mathematics, vol. 170, Birkhäuser Boston Inc., Boston, MA, 1999.

[27] N. Christopher Phillips, Analogs of Cuntz algebras on $L^{p}$ spaces, arXiv:1201.4196 (2012). 
[28] Crossed products of $L^{p}$ operator algebras and the K-theory of Cuntz algebras on $L^{p}$ spaces, arXiv:1309.6406 (2013).

[29] _ Isomorphism, nonisomorphism, and amenability of $L^{p}$ UHF algebras, arXiv:1309.3694 (2013).

[30] Simplicity of UHF and Cuntz algebras on $L^{p}$-spaces, arXiv:1309.0115 (2013).

[31] N. Christopher Phillips and Maria Grazia Viola, $L^{p}$ analogs of AF algebras, in preparation.

[32] Gilles Pisier, Completely bounded maps between sets of Banach space operators, Indiana University Mathematics Journal 39 (1990), no. 1, 249-277.

[33] Sanaz Pooya and Shirin Hejazian, Simple reduced $L^{p}$ operator crossed products with unique trace, arXiv:1402.3233 (2014).

[34] Ian F. Putnam, The $C^{*}$-algebras associated with minimal homeomorphisms of the Cantor set, Pacific Journal of Mathematics 136 (1989), no. 2, 329-353.

[35] Arlan Ramsay, Virtual groups and group actions, Advances in Mathematics 6 (1971), no. 3, 253-322.

[36] Topologies on measured groupoids, Journal of Functional Analysis 47 (1982), no. 3, 314-343.

[37] Jean Renault, A Groupoid Approach to $C^{*}$-algebras, Lecture Notes in Mathematics, vol. 793, Springer, Berlin, 1980.

[38] — $C^{*}$-algebras and dynamical systems, Publicações Matemáticas do IMPA, Instituto Nacional de Matemática Pura e Aplicada, Rio de Janeiro, 2009.

[39] Mikael Rørdam, Flemming Larsen, and Niels Laustsen, An introduction to K-theory for $C^{*}$-algebras, London Mathematical Society Student Texts, vol. 49, Cambridge University Press, Cambridge, 2000.

[40] Dana P. Williams, Crossed Products of $C^{*}$-algebras, Mathematical Surveys and Monographs, vol. 134, American Mathematical Society, Providence, RI, 2007.

Eusebio Gardella, Department of Mathematics, Deady Hall, University of Oregon, Eugene OR 97403-1222, USA, And Fields Institute for Research in Mathematical Sciences, 222 College Street, Toronto ON M5T 3J1, Canada. E-mail address: gardella@uoregon.edu

$U R L:$ http://pages.uoregon.edu/gardella/

Martino Lupini, Department of Mathematics and Statistics, N520 Ross, 4700 Keele Street, Toronto Ontario M3J 1P3, Canada, and Fields Institute for Research in Mathematical Sciences, 222 College Street, Toronto ON M5T 3J1, CANADA.

E-mail address: mlupini@mathstat.yorku.ca

$U R L:$ http://www.lupini.org/ 